TRANSACTIONS OF THE

AMERICAN MATHEMATICAL SOCIETY

Volume 360, Number 7, July 2008, Pages 3493-3539

S 0002-9947(08)04404-8

Article electronically published on February 13, 2008

\title{
A PRIORI BOUNDS, NODAL EQUILIBRIA AND CONNECTING ORBITS IN INDEFINITE SUPERLINEAR PARABOLIC PROBLEMS
}

\author{
NILS ACKERMANN, THOMAS BARTSCH, PETR KAPLICKÝ, AND PAVOL QUITTNER
}

\begin{abstract}
We consider the dynamics of the semiflow associated with a class of semilinear parabolic problems on a smooth bounded domain, posed with homogeneous Dirichlet boundary conditions. The distinguishing feature of this class is the indefinite superlinear (but subcritical) growth of the nonlinearity at infinity. We present new a priori bounds for global semiorbits that enable us to give dynamical proofs of known and new existence results for equilibria. In addition, we can prove the existence of connecting orbits in many cases.

One advantage of our approach is that the parabolic semiflow is naturally order preserving, in contrast to pseudo-gradient flows considered when using variational methods. Therefore we can obtain much information on nodal properties of equilibria that was not known before.
\end{abstract}

\section{INTRODUCTION}

We consider the parabolic problem

$$
\left\{\begin{aligned}
u_{t}-\Delta u & =\lambda u+a(x) g(u)+h(x, u), & & x \in \Omega, t>0, \\
u & =0, & & x \in \partial \Omega, t>0, \\
u(x, 0) & =u_{0}(x), & & x \in \Omega,
\end{aligned}\right.
$$

for $u=u(x, t)$ and a smooth bounded domain $\Omega \subset \mathbb{R}^{N}$. Here $\lambda \in \mathbb{R}, a \in L^{\infty}(\Omega)$ may change sign, $g \in C^{1}(\mathbb{R}, \mathbb{R})$ is superlinear and subcritical, and $h: \Omega \times \mathbb{R} \rightarrow \mathbb{R}$ has at most linear growth in $u \in \mathbb{R}$ as $|u| \rightarrow \infty$. We require $g(0)=0$ and $h(., 0) \equiv 0$ so that $u \equiv 0$ is a (trivial) solution.

In recent years a number of papers appeared concerning the existence of stationary solutions of $\left(P_{\lambda}\right)$, i.e. solutions of the elliptic problem

$$
\left(E_{\lambda}\right) \quad\left\{\begin{aligned}
-\Delta u & =\lambda u+a(x) g(u)+h(x, u), & & x \in \Omega, \\
u & =0, & & x \in \partial \Omega,
\end{aligned}\right.
$$

where $u=u(x)$. Most papers deal with the existence of positive solutions of $E_{\lambda}$ using either fixed point arguments based on a priori estimates as e.g. Berestycki, Cappuzzo-Dolcetta and Nirenberg [13, Chen and Li [19], or variational methods as

Received by the editors July 29, 2004 and, in revised form, April 7, 2006.

2000 Mathematics Subject Classification. Primary 37L05; Secondary 35K20, 35K55, 37L10, $47 \mathrm{H} 20$.

The first and second authors were supported by DFG Grants BA 1009/15-1, BA 1009/15-2.

The third author was supported by the GACR Grant 201/03/0934.

The fourth author was supported by the DFG Grant Gi 30/76-1.

(C)2008 American Mathematical Society 
Alama and Tarantello [3, Berestycki, Cappuzzo-Dolcetta and Nirenberg [14. Let $0<\lambda_{1}<\lambda_{2}<\lambda_{3}<\ldots$ denote all distinct Dirichlet eigenvalues of $-\Delta$ on $\Omega$ and

$$
\begin{aligned}
& \lambda^{+}:=\sup \left\{\lambda: E_{\lambda} \text { possesses a positive solution }\right\}, \\
& \lambda^{-}:=\sup \left\{\lambda: E_{\lambda} \text { possesses a negative solution }\right\} .
\end{aligned}
$$

If $\lambda<\lambda_{1}$, then under various hypotheses on $a, g$ and $h$ the mountain pass theorem yields a positive and a negative solution of $\left(E_{\lambda}\right)$, hence $\lambda^{+}, \lambda^{-} \geq \lambda_{1}$; cf. [3]. Under some additional assumptions on $a$, Alama and Tarantello [3] proved that $\lambda^{+}, \lambda^{-}>$ $\lambda_{1}$ and that $\left(E_{\lambda}\right]$ has at least two positive and two negative solutions if $\lambda_{1}<\lambda<$ $\min \left\{\lambda^{+}, \lambda^{-}\right\}$. In the case $\lambda<\lambda_{1}$ Alama and Del Pino [2] obtained a third nontrivial solution, and in the case $\lambda_{1}<\lambda<\min \left\{\lambda_{2}, \lambda^{+}, \lambda^{-}\right\}$a fifth nontrivial solution. They do not have any nodal information on these "possibly changing-sign" solutions and they have to assume that $a$ has a "thick" zero set, i.e.

$$
\overline{\{x \in \Omega: a(x)>0\}} \cap \overline{\{x \in \Omega: a(x)<0\}}=\varnothing .
$$

This case has also been considered in the recent paper 18 of Chang and Jiang. They obtained a nontrivial solution provided $\lambda$ does not belong to the Dirichlet spectrum of $-\Delta$ in $\Omega$, nor to the Dirichlet spectrum of $-\Delta$ in the interior of $a^{-1}(0)$. If in addition the set of all positive and negative solutions is bounded, then they showed that this solution does change sign. We refer the reader to [5, 21] for sufficient conditions concerning this boundedness assumption. On the other hand, if $a \in C^{2}(\bar{\Omega}), \nabla a(x) \neq 0$ whenever $a(x)=0$, and some additional hypotheses are satisfied, then the existence of a nontrivial solution was proved by Ramos, Terracini and Troestler in [42, but no information on the nodal properties of the solution was provided.

There do not seem to be many papers investigating the long time dynamics associated to $\left(P_{\lambda}\right)$ with an indefinite superlinear nonlinearity. In particular, nothing seems to be known about the dynamics of $\left(P_{\lambda}\right)$ when the initial value changes sign. In this paper we provide results on $\left(P_{\lambda}\right)$ that in addition yield new information about equilibria, especially about equilibria which change sign. These equilibria will be called nodal as opposed to signed equilibria which do not change sign. We obtain three types of results:

(1) We show the existence of nodal solutions of $\left(E_{\lambda}\right)$ without assuming (1.1) or any a priori bound for signed solutions of $\left(\overline{E_{\lambda}}\right)$.

(2) We obtain new multiplicity theorems for nodal solutions of $\left(E_{\lambda}\right)$. For instance, we prove the existence of two or three nodal solutions if $\lambda_{2}<\lambda<$ $\max \left\{\lambda^{+}, \lambda^{-}\right\}$or $\lambda_{2}<\lambda<\min \left\{\lambda^{+}, \lambda^{-}\right\}$, respectively (and we also show that $\lambda^{ \pm}$can be arbitrarily large depending on $\left.a, g, h\right)$.

(3) We prove the existence of connecting orbits between nodal equilibria of $\left(P_{\lambda}\right)$ and the trivial equilibrium $u \equiv 0$.

Observe that nodal equilibria of $\left(P_{\lambda}\right)$ and zero are not ordered, therefore the result of Matano 34] on the existence of connecting orbits does not apply.

There are three main difficulties dealing with $\left(P_{\lambda}\right)$ and $\left(E_{\lambda}\right)$ when the nonlinearity is indefinite superlinear. First, compactness conditions are nontrivial, like the Palais-Smale condition for the energy functional $\Phi \in C^{1}\left(H_{0}^{1}(\Omega)\right)$ associated to $\left[E_{\lambda}\right.$. This problem is even more difficult for $\left(P_{\lambda}\right.$ where one needs a priori bounds for global orbits with bounded energy, not just for Palais-Smale sequences. A second difficulty is that the linking arguments in the indefinite setting are more 
complicated. This can be seen in the paper 42 by Ramos, Terracini and Troestler, for instance. A third major difficulty appears when one wants to prove that a certain solution of $\left(E_{\lambda}\right)$ changes sign. In order to illustrate this problem consider the Dirichlet problem

$$
\left\{\begin{aligned}
-\Delta u & =f(x, u), & & x \in \Omega, \\
u & =0, & & x \in \partial \Omega .
\end{aligned}\right.
$$

Suppose that $f$ satisfies the one-sided Lipschitz condition

$$
\inf _{u \neq v, x \in \Omega} \frac{f(x, u)-f(x, v)}{u-v}>-k \text { for some } k \geq 0 .
$$

Then the operator

$$
u \mapsto K(u):=(-\Delta+k)^{-1}(f(\cdot, u)+k u)
$$

is (strongly) order preserving. The gradient vector field $\nabla \Phi$ associated to the functional $\Phi \in C^{1}(E)$ with respect to a suitably chosen scalar product on $E:=$ $H_{0}^{1}(\Omega)$ has the form $\nabla \Phi=\mathrm{Id}-K$. It follows that one can construct a pseudo gradient flow which leaves the cones $P^{+}:=\{u \in E: u \geq 0$ a.e. $\}$ and $P^{-}:=$ $-P^{+}$positively invariant. This is essential when one wants to find critical points of $\Phi$ outside of $P^{+} \cup P^{-}$as one can see in the recent papers [8, 10, 20, 11, 29] on nodal solutions for (1.2) when (1.3) holds. This approach does not work for indefinite nonlinearities because (1.3) necessarily fails. In [29] Li and Wang suggest a modification of the gradient flow in order to solve this problem. However their idea does not seem to work either: it is based on the claim that the inverse of some operator $A_{0}$ (see p. 390 in [29]) is order-preserving, but this is not true. A different method has been applied by Chang and Jiang in [18. They also use variational arguments (Morse theory, critical groups), but they do not need the positive invariance of the cones $P^{ \pm}$. However, for their approach it is essential that (1.1) is satisfied and the set of signed solutions is bounded.

In 1, 41, (see also the references therein) the authors showed that the parabolic semiflow can be used to find nodal equilibria for definite superlinear problems. A reader who is only interested in solutions of $\left(E_{\lambda}\right.$ may think of the parabolic semiflow as a substitute of the negative gradient flow which has better order preserving properties. However, the parabolic semiflow is interesting in itself since it models the dynamical behavior in many applications coming from natural sciences and engineering. Thus it is well worthwhile to understand the long-time behavior of solutions of $\left(P_{\lambda}\right)$. Due to $g$ being superlinear the parabolic semiflow generated by $\left(\overline{P_{\lambda}}\right)$ is nondissipative and a global attractor does not exist. Quite to the contrary, solutions may (and do) blow up in finite time, a phenomenon which has been widely investigated in recent years. This makes a priori estimates for global solutions difficult to obtain already in the definite case. When the nonlinearity is definite $a$ priori estimates have been obtained by $\mathrm{Ni}$, Sacks and Tavantzis [36, Cazenave and Lions [16], Giga [23] and Quittner [39, 40], but no estimates seem to be available in the indefinite case.

We shall provide the first a priori estimates for global solutions of $\left(P_{\lambda}\right)$ with indefinite nonlinearity. These estimates are of independent interest since such bounds are known to have applications to blow-up [24, 40, to the existence of periodic solutions [4], and to control problems [6]. Unfortunately we need to impose a technical restriction on the growth of $g$. In order to give an idea of our results assume for 
simplicity that $g(u)=|u|^{p-1} u+g_{1}(u)$ where $g_{1} \in C^{1}(\mathbb{R}, \mathbb{R})$ has bounded derivative and $g_{1}(0)=g_{1}^{\prime}(0)=0$, and suppose that $h \equiv 0$. Suppose also that $a \in C^{1}(\Omega)$ changes sign and has 0 as a regular value. Define

$$
p_{\mathrm{S}}:=\frac{N+2}{(N-2)_{+}} \quad \text { and } \quad p_{\mathrm{CL}}:=\frac{3 N+8}{(3 N-4)_{+}},
$$

where $\alpha_{+}$denotes $\max \{\alpha, 0\}$ if $\alpha \in \mathbb{R}$, and $\alpha / 0:=\infty$ if $\alpha>0$. Clearly $p_{\mathrm{S}}$ is the critical Sobolev exponent for the embedding $H_{0}^{1}(\Omega) \hookrightarrow L^{p+1}(\Omega)$, and $p_{\mathrm{CL}}$ is the constant introduced by Cazenave and Lions in [16. We define a new constant $p_{1}^{*} \in\left[p_{\mathrm{CL}}, p_{\mathrm{S}}\right]$ (see (3.3) below) and prove a priori estimates for global solutions of $\left(P_{\lambda}\right.$ provided $p<p_{1}^{*}$. We do not know how to avoid this restriction. On the other hand, $p_{1}^{*}$ is close to $p_{\mathrm{S}}$ for $N$ large in the sense that $p_{\mathrm{S}}-p_{1}^{*}=O\left(1 / N^{3}\right)$ as $N \rightarrow \infty$. In addition, our results remain true for all $p \in\left(1, p_{\mathrm{S}}\right)$ in the radial setting.

We now state some typical results for the model problem

$$
\left\{\begin{aligned}
u_{t}-\Delta u & =\lambda u+a(x)\left(|u|^{p-1} u+g_{1}(u)\right), & & x \in \Omega, t>0, \\
u & =0, & & x \in \partial \Omega, t>0, \\
u(x, 0) & =u_{0}(x), & & x \in \Omega .
\end{aligned}\right.
$$

First we consider a priori bounds for global solutions with bounded energy.

Theorem A. Suppose that $1<p<p_{1}^{*}$.

(a) For all $C_{0}, \eta>0$ there exists $C>0$ with the following property: If $u$ is any global solution of (1.4) satisfying $|\Phi(u(t))| \leq C_{0}$ for all $t>0$, then $\|u(t)\|_{H^{1}(\Omega)} \leq C$ for all $t>\eta$.

(b) Let $u$ be a solution of (1.4) which blows up in a finite time $T$ in the $H^{1}$ norm. Then $\Phi(u(t)) \rightarrow-\infty$ as $t \rightarrow T-$.

Based on these and related a priori bounds we obtain results on equilibria and connecting orbits of (1.4). Typical results are:

Theorem B. Suppose that $1<p<p_{1}^{*}$.

(a) If $\lambda \neq \lambda_{k}$ for every $k \geq 2$, then there exists a nodal equilibrium $u$ and $a$ connecting orbit between $u$ and 0 .

(b) If $\lambda_{2}<\lambda<\min \left\{\lambda^{+}, \lambda^{-}\right\}$, then there exist at least seven nontrivial equilibria: two positive, two negative, three nodal.

(c) If $g_{1}$ is odd, then there exists a sequence of nodal equilibria with unbounded energy. All these equilibria have a connecting orbit to 0 .

(d) If $\lambda_{2}<\lambda<\min \left\{\lambda^{+}, \lambda^{-}\right\}$and if $g_{1}$ is odd, then there exist at least $2(\mu(0)-1)$ nodal equilibria with the following property: if $u$ is any of these equilibria, then there exists a connecting orbit from 0 to $u$. Here $\mu(0)$ denotes the Morse index of 0.

In the case $\lambda=\lambda_{k}$ we can also prove the existence of a nodal equilibrium of $\left(P_{\lambda}\right.$ but we need to assume additional hypotheses if $\lambda>\lambda^{+}$(which we believe to be purely technical). In case (b) of Theorem B we also have results on connecting orbits between (some of) the equilibria. Concerning (c) note that the existence of infinitely many pairs of equilibria is already proved in [3] for odd nonlinearities. The essential new information here is the nodal information and the existence of connecting orbits. 
We would like to emphasize that we consider much more general situations than the one described above: the function $g_{1}$ may be superlinear, $a$ need not be continuous and the zero set may be thick as in (1.1) or it may have measure 0. The precise statement of our results can be found in the following section (see Theorems 2.1. 2.3, 2.6 and Remarks 2.7, 2.8, 2.9 and 2.10).

\section{Statement of Results}

Throughout the paper we fix $N$ in $\mathbb{N}$, a smooth and bounded domain $\Omega \subset \mathbb{R}^{N}$, and denote

$$
E:=H_{0}^{1}(\Omega) \quad \text { and } \quad X:=C^{1}(\bar{\Omega}) \cap E .
$$

We also denote by $L$ the operator $-\Delta$ in $E$, by $\sigma(L)$ its spectrum, and by $0<\lambda_{1}<$ $\lambda_{2}<\ldots$ its distinct eigenvalues.

Let $\mathcal{F}_{\mathrm{S}}$ denote the set of all Carathéodory functions $f: \Omega \times \mathbb{R} \rightarrow \mathbb{R}$ such that $f(\cdot, 0) \in L^{\infty}(\Omega)$ and

$$
|f(x, u)-f(x, v)| \leq C|u-v|\left(1+|u|^{r-1}+|v|^{r-1}\right),
$$

where $C>0$ and $r \in\left[1, p_{\mathrm{S}}\right)$ may depend on $f$. We shall also write $g \in \mathcal{F}_{\mathrm{S}}$ if $g: \mathbb{R} \rightarrow \mathbb{R}$ and the function $\tilde{g}(x, u):=g(u)$ belongs to $\mathcal{F}_{\mathrm{S}}$.

Now consider functions $a, g$ and $h$ as in $\left(P_{\lambda}\right)$. We define

$$
G(u):=\int_{0}^{u} g(s) d s, \quad H(x, u):=\int_{0}^{u} h(x, s) d s,
$$

and

$$
\begin{aligned}
\Omega^{+} & :=\{x \in \Omega: a(x)>0\}, \\
\Omega^{0} & :=\{x \in \Omega: a(x)=0\}, \\
\Omega^{-} & :=\{x \in \Omega: a(x)<0\} .
\end{aligned}
$$

If int $\Omega^{0}$, the interior of $\Omega^{0}$, is a Lipschitz domain, then we denote by $L_{0}$ the operator $-\Delta$ in $H_{0}^{1}\left(\right.$ int $\left.\Omega^{0}\right)$ and by $\sigma\left(L_{0}\right)$ its spectrum.

In Section 3 we define constants $p_{i}^{*}=p_{i}^{*}(N), i=1,2$, such that $(N+3) /(N-1) \leq$ $p_{2}^{*} \leq p_{1}^{*} \leq p_{\mathrm{S}}$ and $p_{1}^{*} \geq p_{\mathrm{CL}} ;$ see (3.3) and (3.51).

We consider the following assumptions:

(A1) $a \in L^{\infty}(\Omega)$, the sets $\Omega^{+}, \Omega^{-}$are open and $\Omega^{+} \neq \varnothing$. In addition, $g, h \in$ $\mathcal{F}_{\mathrm{S}}, g \in C^{1}(\mathbb{R}, \mathbb{R})$, the derivative $h_{u}(x, u)$ exists for all $(x, u)$ and $h_{u}(x, \cdot)$ is continuous for a.e. $x$.

(A2) There is $C>0$ such that $|h(x, u)| \leq C(1+|u|)$ for all $(x, u) \in \Omega \times \mathbb{R}$.

(A3) There are $p \in\left(1, p_{1}^{*}\right)$ and $C>0$ such that $\left.|g(u)-| u\right|^{p-1} u \mid \leq C(1+|u|)$ for all $u \in \mathbb{R}$.

(A4) If $\lambda \geq \lambda_{1}$, then one of the following holds:

$$
\text { meas } \Omega^{0}=0
$$

or

$$
\begin{aligned}
& \operatorname{int} \Omega^{0} \text { is a Lipschitz domain, meas }\left(\Omega^{0} \backslash \operatorname{int} \Omega^{0}\right)=0, \lambda \notin \sigma\left(L_{0}\right) \text { and } \\
& \lim _{|u| \rightarrow \infty} h(x, u) / u=0 \text { for every } x \in \operatorname{int} \Omega^{0} .
\end{aligned}
$$

(A5) $g(0)=g^{\prime}(0)=0$, and $h(x, u)=o(|u|)$ as $u \rightarrow 0$, uniformly in $x$. In addition, the derivatives $g^{\prime}$ and $h_{u}(x, \cdot)$ are Hölder continuous at $u=0$, uniformly in $x$.

(A6) $a \in C^{1}(\bar{\Omega})$ and 0 is a regular value of $a$. 
(A7) $a(x) G(u)+H(x, u) \geq 0$ for small $|u|$.

(A8) $a \in C^{2}(\bar{\Omega}), 0$ is a regular value of $a$ and $a \neq 0$ on $\partial \Omega$. In addition, there are $p \in\left(1, p_{2}^{*}\right)$ and $C>0$ such that

$$
\lim _{|u| \rightarrow \infty} \frac{g(u)}{|u|^{p-1} u}=C .
$$

Let us assume (A1) for the rest of this section. This implies that $\left(P_{\lambda}\right)$ generates a compact continuous (local) semiflow on $E$. If for two equilibria $u_{1}, u_{2} \in E$ there exists a global orbit through $u \in E$ such that $u_{1} \in \alpha(u)$ and $u_{2} \in \omega(u)$, then we say that $u_{1}$ connects to $u_{2}$ and write $u_{1} \succ u_{2}$. Here $\alpha(u)$ and $\omega(u)$ denote the $\alpha$ and $\omega$-limit sets of $u$, respectively.

Define the usual energy functional $\Phi: E \rightarrow \mathbb{R}$ by

$$
\Phi(u):=\frac{1}{2} \int_{\Omega}\left(|\nabla u|^{2}-\lambda u^{2}\right) d x-\int_{\Omega}(a(x) G(u)+H(x, u)) d x .
$$

Then $\Phi \in C^{2}(E, \mathbb{R})$ and the dynamical system defined by $P_{\lambda}$ possesses $\Phi$ as a strict Lyapunov function, i.e.

$$
\Phi\left(u\left(t_{2}\right)\right)<\Phi\left(u\left(t_{1}\right)\right) \quad \text { if } \quad 0 \leq t_{1}<t_{2}<T
$$

for a nonstationary solution $u:[0, T) \rightarrow E$ of $\left(P_{\lambda}\right)$. The solutions of $\left(E_{\lambda}\right)$ coincide with the critical points of $\Phi$. If (A5) is true, then 0 is a critical point of $\Phi$. As usual, for $c \in \mathbb{R}$ denote the corresponding sublevel set of $\Phi$ by

$$
\Phi^{c}:=\{u \in E: \Phi(u) \leq c\} .
$$

Denote by $\Lambda^{+}\left(\Lambda^{-}\right)$the set of $\lambda \in \mathbb{R}$ such that $\left(E_{\lambda}\right)$ admits a positive (negative) solution. Recall that $\lambda^{ \pm}=\sup \Lambda^{ \pm}$. Assuming (A1) (A5) (or (A8) instead of (A3) and (A4) we will show that $\lambda_{1} \leq \lambda^{ \pm}<\infty$, but also that $\lambda^{+}$and $\lambda^{-}$may be arbitrarily large (see Theorem 2.3) (a), Lemma 4.3, Proposition 4.6). In addition, $\left(-\infty, \lambda^{ \pm}\right) \subset \Lambda^{ \pm}$and $\lambda^{+} \in \Lambda^{+}$if $\lambda^{+}>\lambda_{1}$ (similarly for $\lambda^{-}$, see Corollary $[5.10$ and cf. [5]). Replacing $g(u)$ by $-g(-u)$ and $h(x, u)$ by $-h(x,-u)$ if necessary, we may assume without loss of generality that $\lambda^{+} \geq \lambda^{-}$.

We can now state the first main result of the present paper:

Theorem 2.1. Assume (A1) (A5) and $\lambda^{+} \geq \lambda^{-}$. A nodal equilibrium of $\varphi$ exists in the following cases:

(a) $\lambda<\lambda_{2}$.

(b) $\lambda_{2} \leq \lambda \leq \lambda^{+}$.

(c) $\lambda^{+}<\lambda, \lambda \notin \sigma(L)$ and $(\mathrm{A} 6)$ holds.

(d) $\lambda^{+}<\lambda, \lambda \in \sigma(L)$, (A7) and (A6) hold.

We can also state about connections with 0 that a nodal equilibrium $u$ exists such that $u \succ 0$ in case (a); $0 \succ u$ in case (b) with $\lambda_{2}<\lambda$; $u \succ 0$ or $0 \succ u$ in case (c). and either $\Phi(u) \leq 0$ or $u \succ 0$ in case $(\mathrm{d})$.

Remark 2.2. If $\lambda=\lambda_{2} \leq \lambda^{+}$, then Theorem 2.1 does not yield any information about connecting orbits between the nodal equilibrium and zero. However, if we assume that $\Phi \leq 0$ on the eigenspace of $L$ corresponding to the spectral set $\left\{\lambda_{1}, \lambda_{2}\right\}$, then we can argue as in [1] to obtain a nodal equilibrium that connects to 0 . A similar integral condition has been used in [25, 32].

Our second main result concerns multiplicity of equilibria. 
Theorem 2.3. Assume (A1) (A5) and $\lambda^{+} \geq \lambda^{-}$.

(a) If $\lambda<\lambda_{1}$ there exist at least three nontrivial equilibria that connect to 0 : one positive, one negative, and one nodal.

(b) If $\lambda_{1}<\lambda<\lambda^{+}$there exist at least three nontrivial equilibria: two positive and one nodal.

(c) If $\lambda_{1}<\lambda<\lambda^{-}$there exist at least five nontrivial equilibria: two positive, two negative, and one nodal.

(d) If $\lambda_{2}<\lambda<\lambda^{+}$there exist at least four nontrivial equilibria: two positive and two nodal.

(e) If $\lambda_{2}<\lambda<\lambda^{-}$and $\lambda \notin \sigma(L)$ there exist at least seven nontrivial equilibria: two positive, two negative, and three nodal.

Remark 2.4. Instead of assuming $\lambda \notin \sigma(L)$ in Theorem 2.3] (e) it is sufficient to assume that the critical group $C_{\mu}(\Phi, 0) \neq 0$ for some $\mu \geq 2$; cf. [35, 17] and Section 6 below for a definition of the critical groups.

Remark 2.5. We also have some information on the existence of connecting orbits in the statements (b) (e) of Theorem 2.3, see the proofs and Remark 6.8, In addition, the proofs yield information on the Morse type of the solutions, i.e. whether a solution is a local minimum, or of mountain pass type (cf. [26]), or of Morse index 2 type.

Theorem 2.6. Assume (A1) (A5) and that the functions $g$ and $h(x, \cdot)$ are odd.

(a) There exists a sequence of nodal equilibria with unbounded energy $\Phi$ that connect to 0 .

(b) If $\lambda_{2}<\lambda<\lambda^{-}$, then there are at least $2(\mu(0)-1)$ nodal equilibria that 0 connects to. Here $\mu(0)$ denotes the Morse index of 0 .

Remark 2.7. Hypotheses (A3) and (A4) in Theorems 2.1, 2.3, 2.6 can be replaced by assumption (A8). This follows from the proofs of those theorems and from Theorem 3.13 below. Notice that (A8) allows us to consider superlinear perturbations of the power function $|u|^{p-1} u$.

Remark 2.8. If $\Omega$ is a ball or an annulus, the functions $a$ and $h(\cdot, u)$ are radially symmetric and if we restrict ourselves to radial solutions, then the condition $p<p_{1}^{*}$ in (A3) (and $p<p_{2}^{*}$ in (A8) may be replaced by $p<p_{\mathrm{S}}$. This follows from Corollary 3.15 and its proof. Of course, in this case we have to consider just those eigenvalues $\lambda_{i}$ which possess radial eigenfunctions.

Remark 2.9. We do not assume that $\Omega^{-} \cup \Omega^{0} \neq \varnothing$. Therefore the results stated above are also true for definite superlinear equations, and some of them are new even in that setting. In addition, if $a$ is positive and bounded away from zero, then the condition $p<p_{1}^{*}$ (or $p<p_{2}^{*}$ ) may be replaced by $p<p_{\mathrm{S}}$, due to the estimates in [40].

Remark 2.10. The upper bound $p_{1}^{*}$ for the exponent $p$ in (A3) is greater than the exponent $p_{\mathrm{CL}}$ whenever $N>2$. If $N=2$, then under some additional assumptions on $a$ and/or $\lambda$ we can prove many of our results for some $p>p_{\mathrm{CL}}$ as well; see Theorem 3.16 and Remark 3.17 .

The paper is organized as follows. Section 3 is devoted to a priori estimates of global solutions of $\left(P_{\lambda}\right)$ and other auxiliary results. In Section 4 we study 
the existence of signed solutions of $E_{\lambda}$. If $\lambda>\lambda_{2}$ and if there exist a positive supersolution $\bar{u}$ and a negative subsolution $\underline{u}$ of $\left(E_{\lambda}\right)$, then the existence of multiple nodal solutions between $\underline{u}$ and $\bar{u}$ is established in Section [6. Nodal solutions lying outside the order interval $[\underline{u}, \bar{u}]$ (and their connections to the zero solution) are found in Section 5. In that section we also prove the existence of nodal equilibria in the case when no positive supersolutions (or negative subsolutions) exist or when $\lambda<\lambda_{2}$. Finally, in Section $\mathrm{A}$ we provide a technical result that is claimed and used in [1] without proof.

The theorems stated above are consequences of many particular results that are scattered throughout the paper. For the reader's convenience we now show which results have to be used in their proofs.

Proof of Theorem 2.1. Part (a) is exactly the statement of Proposition 5.6. Assertion (b) is a consequence of Proposition 5.12 and Corollary 5.10. Parts (c) and (d) follow by Proposition 5.7 (a) and (b). Note that in this case the solution must be nodal as $\lambda>\lambda^{+}$.

Proof of Theorem [2.3. In case (a) Proposition [5.5yields positive and negative equilibria $u^{ \pm}$of $\left(P_{\lambda}\right)$ such that $u^{ \pm} \succ 0$. Existence of a nodal equilibrium $u^{*} \succ 0$ follows by Proposition [5.6.

Parts (b), (c) and (d) follow from Corollary 5.10, Corollary 5.11 and Proposition 5.12 In case (e) it follows as in the beginning of the proof of Corollary 5.10 that (6.2) holds. Therefore we can apply Propositions 6.3 and 6.4 in addition to the statements made above. Note in this respect that $C_{k}(\Phi, 0) \neq 0$ by [17, Theorem I.4.1] if $\lambda_{k}<\lambda<\lambda_{k+1}$.

Proof of Theorem 2.6. The first part is a consequence of Proposition 5.13 and the second part follows from Proposition 6.9.

2.1. General notation. We set $\mathbb{R}^{+}:=(0, \infty)$ and $\mathbb{R}_{0}^{+}:=[0, \infty)$. By $C_{c}^{\infty}(U)$ we denote the real $C^{\infty}$-functions on an open subset $U$ of $\mathbb{R}^{N}$ with compact support in $U$. Denote by $q^{\prime}:=q /(q-1)$ the conjugate exponent of $q$ if $q>1$. By $\|\cdot\|_{s, q}$, $s>1 / q-1, q>1$, we denote the norm in $W^{s, q}(\Omega)$, where $W^{s, q}(\Omega)$ is the standard Sobolev-Slobodeckii space if $s \geq 0$ and it is the dual of the Sobolev-Slobodeckii space $W^{-s, q^{\prime}}(\Omega)$ if $s<0$. By $\|\cdot\|_{q}$ we denote the norm in $L^{q}(\Omega), q \geq 1$. As usual we denote by $H_{0}^{1}(\Omega)$ the closure of $C_{c}^{\infty}(\Omega)$ in $H^{1}(\Omega)=W^{1,2}(\Omega)$.

For a topological vector space $Y$ of real functions we denote by $\mathcal{P} Y$ the cone of functions taking values in $\mathbb{R}_{0}^{+}$. The interior of $\mathcal{P} Y$ will be denoted by $\mathcal{P}_{0} Y$. In our setting we use the notation

$$
\begin{aligned}
u \geq v & : \Leftrightarrow u-v \in \mathcal{P} H^{1}(\Omega) \\
u>v & : \Leftrightarrow u-v \in \mathcal{P} H^{1}(\Omega) \backslash\{0\}, \\
u \gg v & : \Leftrightarrow u-v \in \mathcal{P}_{0}\left(C^{1}(\bar{\Omega}) \cap H_{0}^{1}(\Omega)\right) .
\end{aligned}
$$

If $\varphi$ is a continuous (local) semiflow on a metric space $Y$, denote by $T_{+}: Y \rightarrow$ $(0, \infty]$ the maximal existence time for $\varphi$. The domain of $\varphi$ is given by

$$
\mathcal{D}:=\left\{(t, u) \in \mathbb{R}_{0}^{+} \times Y: 0 \leq t<T_{+}(u)\right\},
$$

and for $t \geq 0$ we also set

$$
\mathcal{D}_{t}:=\left\{u \in Y: t<T_{+}(u)\right\} .
$$


Note that $\mathcal{D}$ is open in $\mathbb{R}_{0}^{+} \times Y$ and $\mathcal{D}_{t}$ is open in $Y$. For every $t \geq 0$ we write the time-t-map as $\varphi^{t}: \mathcal{D}_{t} \rightarrow Y$ and we set $\varphi^{-t}:=\left(\varphi^{t}\right)^{-1}$.

For $A \subset Y$ we define its positive semiorbit, its negative semiorbit, and its orbit by

$$
\begin{aligned}
\mathcal{O}_{+}(A) & :=\bigcup_{t \geq 0} \varphi^{t}\left(\mathcal{D}_{t} \cap A\right), \\
\mathcal{O}_{-}(A) & :=\bigcup_{t \geq 0} \varphi^{-t}(A), \\
\mathcal{O}(A) & :=\mathcal{O}_{+}(A) \cup \mathcal{O}_{-}(A),
\end{aligned}
$$

respectively. We also write $\mathcal{O}(u):=\mathcal{O}(\{u\})$ for the orbit through $u$. We say $A$ is positive invariant if $\mathcal{O}_{+}(A) \subset A$ and $A$ is negative invariant if $\mathcal{O}_{-}(A) \subset A$. We say $A$ is invariant if $A$ is positive and negative invariant.

For a closed subset $A$ of $Y$ we define the set of attraction of $A$ by

$$
\mathcal{A}(A):=\left\{u \in Y: A \subset U \subset Y, U \text { open } \Rightarrow \exists t \geq 0: \mathcal{O}_{+}\left(\varphi^{t}(u)\right) \subset U\right\} .
$$

For one point sets we also write $\mathcal{A}(u)$ instead of $\mathcal{A}(\{u\})$. Moreover, by

$$
\partial \mathcal{A}(A):=\overline{\mathcal{A}(A)} \backslash \mathcal{A}(A)
$$

we denote the boundary of the set of attraction of $A$. Note that if $A$ has a neighborhood that is included in $\mathcal{A}(A)$, then $\mathcal{A}(A)$ is open as a consequence of the continuity of $\varphi$.

As usual we define

$$
\alpha(A):=\bigcap_{t \geq 0} \overline{\bigcup_{s \geq t} \varphi^{-s}(A)} \quad \text { and } \quad \omega(A):=\bigcap_{t \geq 0} \overline{\bigcup_{s \geq t} \varphi^{s}\left(\mathcal{D}_{s} \cap A\right)},
$$

the $\alpha$ - and $\omega$-limit sets of $A \subset Y$.

\section{The PARABOliC SEMIFlow AND A PRIORI BOUNDS}

This section is devoted to the study of the parabolic semiflow induced by $\left(P_{\lambda}\right)$, and to a priori estimates of global orbits. To state some of the results in more generality, consider the problem

$$
\left\{\begin{aligned}
u_{t}-\Delta u & =f(x, u), & & x \in \Omega, t>0, \\
u & =0, & & x \in \partial \Omega, t>0, \\
u(x, 0) & =u_{0}(x), & & x \in \Omega .
\end{aligned}\right.
$$

Notice that the problem (3.1) is well posed in $E$ if $f \in \mathcal{F}_{\mathrm{S}}$. In addition, the corresponding local semiflow $\varphi$ in $E$ is compact and $\varphi^{t}: E \rightarrow X$ is continuous for every $t>0$. From the parabolic comparison principle it follows that $u, v \in E$ and $u>v$ imply $\varphi^{t}(u) \gg \varphi^{t}(v)$ if $t$ is such that $\varphi^{t}(u)$ and $\varphi^{t}(v)$ are defined. The solution of (3.1) at time $t$ will also be denoted by $u\left(t ; u_{0}\right)$.

Define

$$
p^{*}:=\frac{9 N^{2}-4 N+16 \sqrt{N(N-1)}}{(3 N-4)^{2}}
$$


and

$$
p_{1}^{*}:= \begin{cases}p_{\mathrm{CL}} & \text { if } N=1,2, \\ \frac{18}{5} & \text { if } N=3, \\ p^{*} & \text { if } N>3 .\end{cases}
$$

Notice that $p_{\mathrm{S}}>p^{*}>p_{\mathrm{CL}}$ if $N>1$ and $p^{*}>p_{1}^{*}>p_{\mathrm{CL}}$ if $N=3$. We will assume that

$$
f \in \mathcal{F}_{\mathrm{S}} \quad \text { and } \quad|f(x, u)| \leq C_{f}\left(1+|u|^{p}\right)
$$

for some $p \in\left(1, p_{1}^{*}\right)$ and $C_{f}>0$. We will also often assume the superlinearity condition

$$
f(x, u) u \geq \theta F(x, u)-\lambda_{F} u^{2}-C_{F}, \quad \theta>2, \lambda_{F}, C_{F} \in \mathbb{R},
$$

where $F(x, u):=\int_{0}^{u} f(x, s) d s$, and the following condition on the initial data $u_{0}$ :

$$
u \text { is a global solution of (3.1), } \quad\left|\Phi\left(u_{0}\right)\right| \leq C_{0},
$$

where

$$
\Phi(u):=\frac{1}{2} \int_{\Omega}|\nabla u|^{2} d x-\int_{\Omega} F(x, u) d x
$$

is the associated energy functional. In some of our assertions we will also assume that

$$
\Phi(u(t)) \geq-C_{\Phi} \quad \text { for every } t \geq 0,
$$

and

$$
\int_{\Omega} u^{2}(x, t) d x \leq C_{L 2} \quad \text { for every } t \geq 0 .
$$

By $c, C$ we denote generic constants which may depend on $\Omega, N, p, C_{f}, C_{F}, \theta$, $\lambda_{F}, C_{0}$ (and on $C_{\Phi}$ or $C_{L 2}$ if (3.7) or (3.8) are assumed, respectively) and which may change from step to step but which are independent of $u_{0}$. Fixed constants depending on the parameters mentioned above will be denoted by $c_{1}, C_{1}, c_{2}, C_{2}, \ldots$.

Lemma 3.1. Assume $f \in \mathcal{F}_{\mathrm{S}}$, (3.6) and (3.7). Then

$$
|\Phi(u(t))| \leq C
$$

and

$$
\int_{0}^{\infty} \int_{\Omega} u_{t}^{2} d x d t \leq C .
$$

Proof. Bound (3.9) follows from (3.6), (3.7) and the fact that the function $t \mapsto$ $\Phi(u(t))$ is nonincreasing. Bound (3.10) follows from the estimate

$$
\int_{t_{1}}^{t_{2}} \int_{\Omega} u_{t}^{2} d x d t \leq \Phi\left(u\left(t_{1}\right)\right)-\Phi\left(u\left(t_{2}\right)\right), \quad t_{2}>t_{1} \geq 0 .
$$

Theorem 3.2. Assume (3.4) with $p \in\left(1, p_{1}^{*}\right)$ and (3.5). Then, given $\eta>0$, there exists $C=C(\eta)$ such that

$$
\|u(t)\|_{1,2} \leq C \quad \text { for every } t>\eta,
$$

for all solutions $u$ satisfying (3.6), (3.7) and (3.8). 
Proof. Let $u$ be a solution satisfying (3.6), (3.7) and (3.8). Multiplying the equation in (3.1) by $u$, integrating over $\Omega$, using (3.5), (3.8) and $\Phi(u(t)) \leq \Phi\left(u_{0}\right) \leq C$, we get

$$
\begin{aligned}
& \frac{1}{2} \frac{d}{d t} \int_{\Omega} u^{2} d x=-\int_{\Omega}|\nabla u|^{2} d x+\int_{\Omega} f(x, u) u d x \\
& \quad \geq-\int_{\Omega}|\nabla u|^{2} d x+\theta \int_{\Omega} F(x, u) d x-\lambda_{F} \int_{\Omega} u^{2} d x-C_{F}|\Omega| \\
& \quad \geq c \int_{\Omega}|\nabla u|^{2} d x-\theta \Phi(u(t))-C \\
& \quad \geq c \int_{\Omega}|\nabla u|^{2} d x-C,
\end{aligned}
$$

where $c:=\theta / 2-1>0$. This estimate, Cauchy's inequality and (3.8) imply

$$
\left(\int_{\Omega}|\nabla u|^{2} d x\right)^{2} \leq C\left(1+\left|\int_{\Omega} u u_{t} d x\right|\right)^{2} \leq C\left(1+\int_{\Omega} u_{t}^{2} d x\right)
$$

hence (3.10) guarantees

$$
\int_{t}^{t+\eta}\left(\int_{\Omega}|\nabla u|^{2} d x\right)^{2} d t \leq C_{1} \quad \text { for every } t \geq 0 .
$$

In particular, there exists $t_{1} \in[0, \eta]$ such that

$$
\left\|u\left(t_{1}\right)\right\|_{1,2}^{4} \leq C_{1} / \eta \text {. }
$$

Interpolation between (3.10) and (3.14) yields a uniform bound for $u(t)$ in $L^{r}(\Omega)$ whenever $r<6 N /(3 N-4)$; see [16].

If $N \leq 2$, then $p<p_{\mathrm{CL}}$ and we can fix $r$ such that $p<1+2 r / N$. Since $\|u(t)\|_{r} \leq C_{r}$ and (3.15) is true, the bound (3.11) follows from [38], for example.

Next assume that $N \geq 3$. Considering the solution $u$ on the interval $\left[t_{1}, \infty\right)$ instead of $[0, \infty)$ we may assume that

$$
\left\|u_{0}\right\|_{1,2} \leq C_{2} .
$$

Standard regularity results imply $u(t) \in C^{1}(\bar{\Omega})$ for every $t>0$. More careful (but straightforward) estimates based on the variation-of-constants formula guarantee the existence of $\delta>0$ and $C_{3}>0$ depending only on the constant $C_{2}$ in (3.16) (and other fixed data of the problem) such that $\|u(t)\|_{1,2} \leq C_{3}$ for every $t \in[0, \delta]$ and $\|u(t)\|_{C^{1}(\bar{\Omega})} \leq C_{3}$ for every $t \in[\delta, 2 \delta]$. Considering the solution $u$ on the interval $[\delta, \infty)$ instead of $[0, \infty)$ we may assume that $u_{0} \in C^{1}(\bar{\Omega})$ and

$$
\|u(t)\|_{C^{1}(\bar{\Omega})} \leq C_{3} \quad \text { for every } t \in[0, \delta] .
$$

Fix $T>\delta+1$ and set

$$
D_{1, \infty}=D_{1, \infty}\left(u_{0}, T\right):=\max \left\{1, \max _{t \in[0, T]}\|u(t)\|_{C^{1}(\bar{\Omega})}\right\} .
$$

For $r>1$ let us also denote

$$
D_{r}=D_{r}\left(u_{0}, T\right):=\max \left\{1, \max _{t \in[0, T]}\|u(t)\|_{r}\right\} .
$$

We already know that $D_{r}<C$ for some $C$ independent of $u_{0}$ and $T$ provided $r<6 N /(3 N-4)$. We want to show by a bootstrap argument that the same is true for $D_{1, \infty}$. More precisely, we will show

$$
D_{1, \infty} \leq C D_{1, \infty}^{\kappa}, \text { where } C>0 \text { and } \kappa<1 \text { are independent of } u_{0} \text { and } T \text {. }
$$


Consider $\varepsilon>0$ small (to be specified later). Since $p<18 / 5$ if $N=3$ and $p<p_{\mathrm{S}}$ if $N>3$, we have $p<6 N /(3 N-4)$ for every $N \geq 3$. In our first bootstrap procedure we will show that given $r \in(p, p+1), r>2$, there exists $\tilde{r}>r$ such that

$$
D_{\tilde{r}} \leq C D_{r}^{(p+1) / 2} D_{1, \infty}^{\varepsilon / 2},
$$

where $C$ may depend on $\varepsilon$. Moreover, the difference $\tilde{r}-r$ can be estimated below by a positive constant which is independent of $r$ and $\varepsilon$. Consequently, starting with some $r \in(2,6 N /(3 N-4)) \cap(p, p+1)$, and using $D_{r}<C$, after finitely many (say $\left.k_{1}\right)$ steps we obtain

$$
D_{p+1} \leq C D_{1, \infty}^{\varepsilon M_{1}}, \quad M_{1}:=\frac{1}{2} \sum_{i=0}^{k_{1}-1}\left(\frac{p+1}{2}\right)^{i} .
$$

Notice that the boundedness of the energy implies

$$
D_{1,2}:=\max \left\{1, \max _{t \in[0, T]}\|u(t)\|_{1,2}\right\} \leq C D_{p+1}^{(p+1) / 2} .
$$

Another (standard) bootstrap procedure easily guarantees

$$
\max _{t \in[\delta, T]}\|u(t)\|_{C^{1}(\bar{\Omega})} \leq C D_{1,2}^{M_{2}}
$$

where $M_{2}$ is a positive constant depending on $p$. Due to (3.17), estimates (3.22), (3.21) and (3.20) guarantee (3.18) if we choose $\varepsilon$ such that $\kappa:=\varepsilon M_{1} M_{2}(p+1) / 2<1$.

Let us prove (3.19). Fix $r \in(p, p+1), r>2$. Set

$$
\nu:=\frac{r}{p}-1, \quad \theta:=\frac{1+\nu}{1-\nu} \cdot \frac{r-2}{r}, \quad r^{\prime}:=\frac{r}{r-1},
$$

and fix $\eta \in\left(0, \varepsilon^{2}\right]$. Then $\nu<1 /(r-1), \theta \in(0,1)$ and using (3.13) and interpolation we obtain (cf. 39, (24)] and [40, Remark 2.5(ii)])

$$
\begin{aligned}
\|u(t)\|_{1,2}^{2} & \leq C\left(1+\left\|u(t) u_{t}(t)\right\|_{1}\right) \leq C\left(1+\|u(t)\|_{\eta, r}\left\|u_{t}(t)\right\|_{-\eta, r^{\prime}}\right) \\
& \leq C\left(1+\|u(t)\|_{\sqrt{\eta}, r}^{\sqrt{\eta}}\|u(t)\|_{r}^{1-\sqrt{\eta}}\left\|u_{t}(t)\right\|_{-\eta, 1+\nu}^{\theta}\left\|u_{t}(t)\right\|_{-\eta, 2}^{1-\theta}\right) \\
& \leq C D_{1, \infty}^{\varepsilon} D_{r}\left(1+\left\|u_{t}(t)\right\|_{-\eta, 1+\nu}^{\theta}\left\|u_{t}(t)\right\|_{2}^{1-\theta}\right) .
\end{aligned}
$$

Standard estimates based on the variation-of-constants formula in extrapolated spaces (see [4]) guarantee the existence of $\omega>0$ such that, given $t \in[\delta, T]$,

$$
\begin{aligned}
\|u(t)\|_{2-\eta, 1+\nu} & \leq \frac{C e^{-\omega t}}{\delta^{1-\eta / 2}}\left\|u_{0}\right\|_{1+\nu}+C \int_{0}^{t} \frac{e^{-\omega(t-s)}}{(t-s)^{1-\eta / 2}}\|f(\cdot, u(\cdot, s))\|_{1+\nu} d s \\
& \leq C\left(1+\sup _{s \in(0, T)}\|f(\cdot, u(\cdot, s))\|_{1+\nu}\right) \\
& \leq C\left(1+\sup _{s \in(0, T)}\|u(s)\|_{p(1+\nu)}^{p}\right) \leq C D_{r}^{p}
\end{aligned}
$$

due to $p(1+\nu)=r$. Now using the equation in (3.1) we obtain

$$
\left\|u_{t}(t)\right\|_{-\eta, 1+\nu} \leq\|u(t)\|_{2-\eta, 1+\nu}+\|f(\cdot, u(\cdot, t))\|_{1+\nu} \leq C D_{r}^{p},
$$

hence (3.24) implies

$$
\|u(t)\|_{1,2}^{2} \leq C D_{1, \infty}^{\varepsilon} D_{r}^{p+1}\left(1+\left\|u_{t}(t)\right\|_{2}^{1-\theta}\right) .
$$

Set

$$
q=q(r):=\frac{2}{1-\theta}=\frac{(1-\nu) r}{1+\nu-\nu r}=\frac{2 p-r}{p+1-r} .
$$


Raising (3.25) to the power $q$, integrating over $(t, t+1) \subset(\delta, T)$ and using (3.10) we obtain

$$
\int_{t}^{t+1}\|u(s)\|_{1,2}^{2 q} d s \leq C D_{1, \infty}^{\varepsilon q} D_{r}^{(p+1) q}\left(1+\int_{t}^{t+1}\left\|u_{t}(s)\right\|_{2}^{2} d s\right) \leq C D_{1, \infty}^{\varepsilon q} D_{r}^{(p+1) q},
$$

hence

$$
\left(\int_{t}^{t+1}\|u(s)\|_{1,2}^{2 q} d s\right)^{1 / 2 q} \leq C D_{1, \infty}^{\varepsilon / 2} D_{r}^{(p+1) / 2} .
$$

Interpolating between (3.27) and (3.10) (see [16] and cf. [39, (11)]) yields

$$
\sup _{s \in[t, t+1]}\|u(s)\|_{\tilde{r}} \leq C D_{1, \infty}^{\varepsilon / 2} D_{r}^{(p+1) / 2},
$$

provided

$$
\tilde{r}<\frac{2 N(q+1)}{q(N-2)+N}
$$

Since $t \in[\delta, T-1]$ was arbitrary and (3.17) is true, we have obtained the desired bound for $D_{\tilde{r}}$. Now (3.26) shows that we can choose $\tilde{r}>r$ if and only if

$$
2(N-1) r^{2}-[(3 N-4) p+5 N] r+2 N(3 p+1)>0 .
$$

It is easy to see that the last inequality is satisfied for every $r$ due to $p<p^{*}$. (On the other hand, that inequality fails for some $r \in(6 N /(3 N-4),(p-1) N / 2)$ if $p \geq p^{*}$.) Consequently, (3.19) is true and the proof is complete.

Remark 3.3. In the proof of Theorem 3.2 we used some ideas from 39] and [40. In fact, if $f \in C^{1}(\bar{\Omega} \times \mathbb{R})$, then we could use a straightforward modification of the proof in [39, pp. 201-202]. However, we need the result for less regular $f$ and this requires some nontrivial changes in that proof. Let us also mention that the a priori bound in 39] (valid for every $p<p_{\mathrm{S}}$ ) required an estimate of the form $\int_{\Omega}|u|^{p+1} d x \leq C \int_{\Omega}|\nabla u|^{2} d x$ which is not available in the present situation. Due to this fact we had to replace the condition $p<p_{\mathrm{S}}$ with $p<p_{1}^{*}$. Notice that the exponent $p^{*}$ is "significantly" greater than $p_{\mathrm{CL}}$. In fact, $p_{\mathrm{S}}-p^{*}<\frac{1}{2}\left(p_{\mathrm{S}}-p_{\mathrm{CL}}\right)$ for $N>2$ and

$$
p_{\mathrm{S}}-p^{*}=O\left(N^{-3}\right), \quad p_{\mathrm{S}}-p_{\mathrm{CL}}=O\left(N^{-2}\right), \quad N \rightarrow \infty .
$$

Remark 3.4. We can choose $\eta=0$ in Theorem 3.2 if we know that $\left\|u_{0}\right\|_{1,2} \leq C$.

Remark 3.5. Replacing the assumption $p<p_{1}^{*}$ by $p<p_{\mathrm{S}}$ in Theorem 3.2 and using the approach from [16] one can still prove that every global solution is bounded. However, the corresponding bound depends on $u_{0}$ and cannot be used in our applications.

The a priori bound (3.11) in Theorem 3.2 is based on the weaker a priori bound (3.8). In what follows we derive sufficient conditions for (3.8).

Theorem 3.6. Assume $f \in \mathcal{F}_{\mathrm{S}}$ and (3.5) with

$$
\lambda_{F}<\lambda_{1}\left(\frac{\theta}{2}-1\right) .
$$

Then the bounds (3.8) and (3.7) are true for all solutions u satisfying (3.6). 
Proof. We will use the concavity argument due to H.A. Levine 28]. Denote $M(t):=$ $\frac{1}{2} \int_{0}^{t} \int_{\Omega} u^{2} d x d t$. Using (3.28), we obtain similarly as in (3.12)

$$
\begin{aligned}
M^{\prime \prime}=\frac{1}{2} \frac{d}{d t} \int_{\Omega} u^{2} d x & \geq-\theta \Phi(u(t))+c_{1} \int_{\Omega}|\nabla u|^{2}(x, t) d x-C_{1} \\
& \geq-\theta \Phi\left(u_{0}\right)+c_{1} \lambda_{1} \int_{\Omega} u^{2}(x, t) d x-C_{1}
\end{aligned}
$$

for some $c_{1}, C_{1}>0$. Assume that

$$
\theta \Phi\left(u\left(t_{0}\right)\right) \leq-C_{1}-1 \quad \text { or } \quad c_{1} \lambda_{1} \int_{\Omega} u^{2}\left(x, t_{0}\right) d x \geq C_{1}+\theta \Phi\left(u_{0}\right)+1
$$

for some $t_{0}>0$. Then (3.29) implies $\frac{1}{2} \frac{d}{d t} \int_{\Omega} u^{2}(x, t) d x \geq 1$ for every $t \geq t_{0}$, hence

$$
\lim _{t \rightarrow \infty} M^{\prime}(t)=\lim _{t \rightarrow \infty} \frac{1}{2} \int_{\Omega} u^{2}(x, t) d x=+\infty .
$$

In particular, there exists $t_{1} \geq t_{0}$ such that

$$
c_{1} \int_{\Omega}|\nabla u|^{2}(x, t) d x>C_{1}+\theta \Phi\left(u_{0}\right) \quad \text { for } t \geq t_{1} .
$$

Consequently, for $t \geq t_{1}$ we obtain

$$
M^{\prime \prime} \geq-\theta \Phi(u(t))+\theta \Phi\left(u_{0}\right) \geq \theta \int_{0}^{t} \int_{\Omega} u_{t}^{2} d x d t,
$$

and

$$
\begin{aligned}
M M^{\prime \prime} & \geq \frac{\theta}{2}\left(\int_{0}^{t} \int_{\Omega} u^{2} d x d t\right)\left(\int_{0}^{t} \int_{\Omega} u_{t}^{2} d x d t\right) \geq \frac{\theta}{2}\left(\int_{0}^{t} \int_{\Omega} u u_{t} d x d t\right)^{2} \\
& =\frac{\theta}{2}\left(M^{\prime}(t)-M^{\prime}(0)\right)^{2} \geq(1+\alpha) M^{\prime}(t)^{2}
\end{aligned}
$$

for some $\alpha>0$ and $t$ large enough due to (3.31). Thus the function $t \mapsto M^{-\alpha}(t)$ is concave, positive and decreasing for $t$ large, which is a contradiction. Hence (3.30) is false and the conclusion follows.

We now turn to the verification of (3.8) in a specialized setting. Therefore assume that

$$
f(x, u)=\lambda u+a(x) g(u)+h(x, u) .
$$

If $g(u)=|u|^{p-1} u, h \equiv 0$ and $a$ changes sign, then the assumption (3.28) in Theorem 3.6 requires $\lambda<\lambda_{1}$. The following theorem guarantees that we may allow $\lambda$ arbitrarily large provided we assume (A4).

Theorem 3.7. Assume (3.32) and (A1) (A4), Then (3.8) is true whenever (3.6) and (3.7) are satisfied.

Proof. Assume on the contrary that there exist $u_{0, k} \in E$ and $t_{k}>0$ such that $\left|\Phi\left(u_{0, k}\right)\right| \leq C_{0}$ and $u_{k}(t):=u\left(t ; u_{0, k}\right)$ satisfy

$$
\inf _{t} \Phi\left(u_{k}(t)\right) \geq-C_{\Phi} \quad \text { and } \quad\left\|u_{k}\left(t_{k}\right)\right\|_{2} \rightarrow \infty .
$$


The boundedness of $\Phi$ implies (3.10) with $u$ replaced by $u_{k}, k=1,2, \ldots$ Using that estimate and Cauchy's inequality we obtain

$$
\begin{gathered}
\int_{\Omega} u_{k}^{2}\left(x, \tau_{2}\right) d x-\int_{\Omega} u_{k}^{2}\left(x, \tau_{1}\right) d x=2 \int_{\tau_{1}}^{\tau_{2}} \int_{\Omega} u_{k} \partial_{t}\left(u_{k}\right) d x d t \\
\geq-C\left(1+\int_{\tau_{1}}^{\tau_{2}} \int_{\Omega} u_{k}^{2} d x d t\right),
\end{gathered}
$$

for every $\tau_{1}<\tau_{2}$. Now by Gronwall's inequality there exists $\eta>0$ such that

$$
\min _{\tau \in\left[t_{k}, t_{k}+\eta\right]} \int_{\Omega} u_{k}^{2}(x, \tau) d x \rightarrow \infty \quad \text { as } k \rightarrow \infty .
$$

Next (3.10) implies the existence of $s_{k} \in\left[t_{k}, t_{k}+\eta\right]$ such that

$$
\int_{\Omega}\left(\partial_{t} u_{k}\right)^{2}\left(x, s_{k}\right) d x \leq C .
$$

Set $L_{k}:=\left\|u_{k}\left(s_{k}\right)\right\|_{2}$. Then $L_{k} \rightarrow \infty$ and

$$
\frac{d}{d t} \int_{\Omega} u_{k}^{2}\left(x, s_{k}\right) d x \leq 2\left(\int_{\Omega} u_{k}^{2}\left(x, s_{k}\right) d x\right)^{1 / 2}\left(\int_{\Omega}\left(\partial_{t} u_{k}\right)^{2}\left(x, s_{k}\right) d x\right)^{1 / 2} \leq C L_{k} .
$$

Since (A1) (A2) and (A3) imply (3.5) with $\theta:=p+1$ and suitable $\lambda_{F}, C_{F}>0$, we obtain similarly as in the proof of Theorem 3.2

$$
\begin{aligned}
\frac{1}{2} \frac{d}{d t} \int_{\Omega} u_{k}^{2} d x & \geq-\int_{\Omega}\left|\nabla u_{k}\right|^{2} d x+(p+1) \int_{\Omega} F\left(x, u_{k}\right) d x-\lambda_{F} \int_{\Omega} u_{k}^{2} d x-C_{F}|\Omega| \\
& =\frac{p-1}{2} \int_{\Omega}\left|\nabla u_{k}\right|^{2} d x-(p+1) \Phi\left(u_{k}(t)\right)-\lambda_{F} \int_{\Omega} u_{k}^{2} d x-C_{F}|\Omega| .
\end{aligned}
$$

Now (3.34) and the boundedness of $\Phi$ guarantee

$$
\int_{\Omega}\left|\nabla u_{k}\right|^{2}\left(x, s_{k}\right) d x \leq C L_{k}^{2}
$$

if $k$ is large enough.

In what follows, for short we write $u_{k}$ instead of $u_{k}\left(\cdot, s_{k}\right)$. Denote

$$
N_{k}:=\left(\int_{\Omega}\left|\nabla u_{k}\right|^{2} d x\right)^{1 / 2}
$$

and recall from 3.36 that

$$
N_{k} \leq C L_{k}=C\left\|u_{k}\right\|_{2}
$$

Set

$$
\alpha(x):= \begin{cases}\operatorname{dist}\left(x, \partial \Omega^{+}\right), & x \in \Omega^{+}, \\ -\operatorname{dist}\left(x, \partial \Omega^{-}\right), & x \in \Omega^{-} \\ 0 & x \in \Omega^{0} .\end{cases}
$$

Then $\alpha$ is Lipschitz continuous and $\alpha a>0$ outside $\Omega^{0}$. Multiplying the equation

$$
\partial_{t} u_{k}=\Delta u_{k}+\lambda u_{k}+a g\left(u_{k}\right)+h\left(x, u_{k}\right), \quad x \in \Omega,
$$

by $\alpha u_{k}$, integrating over $\Omega$ and using (3.33), (A1), (A2), and (A3) we obtain

$$
\int_{\Omega} \alpha a\left|u_{k}\right|^{p+1} d x=O\left(N_{k}^{2}\right) \quad \text { as } k \rightarrow \infty
$$


We may assume that $u_{k} / N_{k}$ converges weakly in $E$, hence strongly in $L^{p+1}(\Omega)$. If (A4.1) is true, then the corresponding limit $w$ is zero due to (3.39). In particular, $u_{k} / N_{k} \rightarrow 0$ in $L^{2}(\Omega)$ which contradicts (3.37). If (A4.2) is true, then $w=0$ on $\Omega \backslash \Omega^{0}$ due to (3.39), hence $w \in H_{0}^{1}\left(\right.$ int $\left.\Omega^{0}\right)$ due to (A4.2). Since $u_{k}$ solve the equation

$$
\partial_{t} u_{k}=\Delta u_{k}+\lambda u_{k}+h\left(x, u_{k}\right), \quad x \in \operatorname{int} \Omega^{0},
$$

and (A4.2), (3.33) are true, $w$ is a (weak) solution of the equation

$$
0=\Delta w+\lambda w, \quad x \in \operatorname{int} \Omega^{0} .
$$

Since $\lambda \notin \sigma\left(L_{0}\right)$, we have $w=0$ which contradicts (3.37) as before. This concludes the proof.

In the following theorem we derive conditions guaranteeing blow-up of energy for every nonglobal solution of (3.1) (cf. also the corresponding result in [31]). These conditions will be similar to those appearing in Theorems 3.2 and 3.6 but the condition (3.28) in Theorem 3.6 will become superfluous. In particular, the resulting conditions are also weaker than those in Theorems 3.2 and 3.7 .

Theorem 3.8. Assume (3.4) with $p \in\left(1, p_{1}^{*}\right)$ and (3.5). Let $u$ be a solution of (3.1) which blows up in a finite time $T$ in the $H^{1}(\Omega)$-norm. Then $\Phi(u(t)) \rightarrow-\infty$ as $t \rightarrow T-$.

Proof. By $D$ we will denote a generic constant which may depend on $u_{0}$. Assume on the contrary that $\Phi(u(t)) \geq-D$ for every $t<T$. Then

$$
\int_{0}^{T} \int_{\Omega} u_{t}^{2} d x d t \leq D
$$

and Gronwall's inequality easily implies

$$
\int_{\Omega} u^{2}(x, t) d x \leq D \quad \text { for every } t \in[0, T) .
$$

In the same way as in the proof of Theorem 3.2 we now obtain a bound for $\|u(t)\|_{1,2}$, $t \in[0, T)$, which contradicts our assumptions.

Remark 3.9. Assume (3.32). If we only considered positive solutions of (3.1), then under suitable assumptions on the function $a$ we could derive uniform a priori bounds for global solutions with bounded energy (and energy blow-up for nonglobal solutions) for every $p<p_{\mathrm{S}}$. In fact, one has just to modify the proof of 31, Theorem 4.2] (cf. also [5, 13, 19, 21, where the same ideas are used in order to prove a priori bounds for positive stationary solutions of (3.1)).

In what follows we will assume (3.32) and we denote

$$
\begin{aligned}
\Omega_{\varepsilon}^{+} & :=\{x \in \Omega: a(x)>\varepsilon\}, \\
\Omega_{\varepsilon}^{0} & :=\{x \in \Omega: a(x)=\varepsilon\}, \\
\Omega_{\varepsilon}^{-} & :=\{x \in \Omega: a(x)<\varepsilon\} .
\end{aligned}
$$

Lemma 3.10. Assume (3.32), (A1), (A2), $a \in C(\bar{\Omega})$ and let the estimate in (A3) be true for some $p>1$. Let $u_{0} \in E \cap L^{\infty}(\Omega),\left|u_{0}\right| \leq K, \varepsilon>0$, and let $T$ denote the maximal existence time of the solution $u=u\left(x, t ; u_{0}\right)$. Then there exists a positive constant $C=C(K, \varepsilon)$ such that $|u(x, t)| \leq C$ for every $x \in \Omega_{-\varepsilon}^{-}$and every $t \in[0, T)$. 
Proof. Fix $C_{1}>0$ such that

$$
g(u) \geq \frac{1}{2} u^{p} \geq \frac{4}{\varepsilon}(|\lambda| u+|h(x, u)|) \quad \text { for every } x \in \Omega, u \geq C_{1} .
$$

Then

$$
f(x, u)=\lambda u+a(x) g(u)+h(x, u) \leq-\frac{\varepsilon}{8} u^{p} \quad \text { for every } x \in \Omega_{-\varepsilon / 2}^{-}, \quad u \geq C_{1} .
$$

Consider $\delta>0$ small such that

$$
|a(x)-a(y)| \leq \varepsilon / 2 \quad \text { if }|x-y| \leq \delta
$$

and choose $x_{0} \in \Omega_{-\varepsilon}^{-}$. Then

$$
\Omega_{\delta}\left(x_{0}\right):=\left\{x \in \Omega:\left|x-x_{0}\right|<\delta\right\} \subset \Omega_{-\varepsilon / 2}^{-} .
$$

Let $B_{\delta}:=\left\{x \in \mathbb{R}^{N}:|x|<\delta\right\}$ and let $w=w_{\delta}$ be the unique positive solution of the singular elliptic problem

$$
\Delta w=\frac{\varepsilon}{8} w^{p} \quad \text { in } B_{\delta}, \quad w=\infty \quad \text { on } \partial B_{\delta}
$$

see [33. Then $w_{\delta}(0)=\inf _{B_{\delta}} w_{\delta} \rightarrow \infty$ as $\delta \rightarrow 0+$. Fix $\delta>0$ such that $w_{\delta}(0) \geq$ $\max \left\{C_{1}, K\right\}$. Then the restriction of $w_{\delta}\left(x-x_{0}\right)$ to the set $\Omega_{\delta}\left(x_{0}\right)$ is a supersolution for the corresponding restriction of $u(x, t)$, hence $u\left(x_{0}, t\right) \leq w_{\delta}(0)$ for every $t \in$ $[0, T)$. Since $x_{0} \in \Omega_{-\varepsilon}^{-}$was arbitrary, we obtain a uniform upper bound for $u$ in $\Omega_{-\varepsilon}^{-}$. The lower bound can be obtained in an analogous way.

Lemma 3.11. Assume (3.32) and (A1) (A4), Let $\varepsilon_{k}, \delta_{k} \rightarrow 0$ and let $w_{k}, k=$ $1,2, \ldots$, be solutions of

$$
\left\{\begin{aligned}
-\Delta w & =\left(\lambda-\delta_{k}\right) w+\left(a(x)-\varepsilon_{k}\right) g(w)+h(x, w), & & x \in \Omega, \\
w & =0, & & x \in \partial \Omega .
\end{aligned}\right.
$$

Assume that $\Phi_{k}\left(w_{k}\right)<C$ for some $C>0$, where

$$
\Phi_{k}(w):=\Phi(w)+\frac{\delta_{k}}{2} \int_{\Omega} w^{2} d x+\varepsilon_{k} \int_{\Omega} G(w) d x .
$$

Then the sequence $\left(w_{k}\right)$ is bounded in $E$.

Proof. Assume on the contrary that (for a subsequence) $\left\|w_{k}\right\|_{1,2} \rightarrow \infty$ as $k \rightarrow \infty$. Multiplying the equation

$$
-\Delta w_{k}=\left(\lambda-\delta_{k}\right) w_{k}+\left(a(x)-\varepsilon_{k}\right) g\left(w_{k}\right)+h\left(x, w_{k}\right)
$$

by $w_{k}$, integrating over $\Omega$ and using $\Phi_{k}\left(w_{k}\right)<C$ we arrive at

$$
\begin{aligned}
& \int_{\Omega}((a\left.\left.-\varepsilon_{k}\right) g\left(w_{k}\right) w_{k}+h\left(x, w_{k}\right) w_{k}\right) d x=\int_{\Omega}\left(\left|\nabla w_{k}\right|^{2}-\left(\lambda-\delta_{k}\right) w_{k}^{2}\right) d x \\
&<2\left(C+\int_{\Omega}\left(\left(a-\varepsilon_{k}\right) G\left(w_{k}\right)+H\left(x, w_{k}\right)\right) d x\right) .
\end{aligned}
$$

As a consequence of (A3) we obtain

$$
g(s) s-2 G(s)=\frac{p-1}{p+1}|s|^{p+1}+O\left(s^{2}\right), \quad|s| \rightarrow \infty,
$$

hence the above estimate implies both

$$
\frac{p-1}{p+1} \int_{\Omega}\left(a-\varepsilon_{k}\right)\left|w_{k}\right|^{p+1} d x \leq C\left(1+\int_{\Omega} w_{k}^{2} d x\right)
$$


and

$$
\int_{\Omega}\left|\nabla w_{k}\right|^{2} d x \leq C\left(1+\int_{\Omega} w_{k}^{2} d x\right) .
$$

Setting $v_{k}:=w_{k} /\left\|w_{k}\right\|_{1,2}$ we may assume that $v_{k} \rightarrow v$ weakly in $E$ and strongly in $L^{p+1}(\Omega)$. As in the proof of Theorem 3.7 we obtain $\int_{\Omega} \alpha a|v|^{p+1} d x=0$ and derive a contradiction.

In the following proposition we will assume that there exist $\varepsilon_{k}>0, \varepsilon_{k} \rightarrow 0$ as $k \rightarrow \infty$, such that

$$
\text { meas } \Omega_{\varepsilon_{k}}^{0}=0, \quad k=1,2, \ldots, \quad \text { and } \quad \text { meas } \Omega^{0}=0 .
$$

Notice that this assumption is trivially satisfied if (A6) holds.

Proposition 3.12. Assume (3.32), (A1) (A3) and (3.44). Assume moreover that $a \in C(\bar{\Omega})$. Let $K \subset E$ be compact,

$$
\Phi\left(u_{0}\right)<0 \text { and } u\left(t, u_{0}\right) \text { be unbounded for every } u_{0} \in K \text {. }
$$

Choose $M \geq 0$. Then there is a continuous map $\eta:[0,1] \times K \rightarrow \Phi^{0} \backslash\{0\}$ such that

$$
\eta(0, u)=u, \quad \eta(1, K) \subset H_{0}^{1}\left(\Omega^{+}\right) \cap \Phi^{-M} .
$$

Proof. First let us prove that for $k$ large enough,

$$
\Phi_{\varepsilon_{k}}\left(u_{0}\right)<0 \text { and } u_{\varepsilon_{k}}\left(t ; u_{0}\right) \text { is unbounded for every } u_{0} \in K,
$$

where

$$
\Phi_{\varepsilon}(u):=\Phi(u)+\varepsilon \int_{\Omega} G(u) d x
$$

and $u_{\varepsilon}\left(t ; u_{0}\right)$ denotes the solution of the problem

$$
\left\{\begin{aligned}
u_{t}-\Delta u & =\lambda u+(a(x)-\varepsilon) g(u)+h(x, u), & & x \in \Omega, t>0, \\
u & =0, & & x \in \partial \Omega, t>0, \\
u(x, 0) & =u_{0}(x), & & x \in \Omega .
\end{aligned}\right.
$$

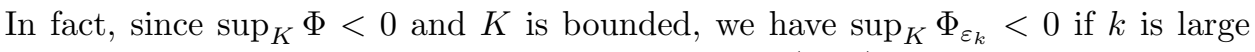
enough. In order to prove the unboundedness of $u_{\varepsilon_{k}}\left(t ; u_{0}\right)$, assume on the contrary that there exist $u_{0, k} \in K$ such that the solutions $u_{k}(t):=u_{\varepsilon_{k}}\left(t ; u_{0, k}\right)$ are global and bounded. Then the $\omega$-limit set of $u_{k}$ is a nonempty set consisting of equilibria. Pick up $w_{k}$ in this set. Since $\Phi_{\varepsilon_{k}}\left(w_{k}\right)<0$, Lemma 3.11 guarantees that the sequence $\left(w_{k}\right)$ is bounded in $E$. Therefore, $\Phi_{\varepsilon_{k}}\left(u_{k}(t)\right) \geq \Phi_{\varepsilon_{k}}\left(w_{k}\right) \geq-C_{\Phi}$ for some $C_{\Phi}>0$. Now a straightforward modification of the proofs in Theorems 3.7 and 3.2 guarantees that there exists a constant $C^{*}>0$ such that

$$
\left\|u_{k}(t)\right\|_{1,2} \leq C^{*} \text { for every } k \text { and every } t \geq 0 .
$$

We may assume that $u_{0, k} \rightarrow u_{0} \in K$. The continuous dependence of the solutions of (3.1) and (3.48) on the initial data and on $\varepsilon$ implies

$$
\left\|u\left(t ; u_{0}\right)\right\|_{1,2} \leq C^{*}
$$

which contradicts our assumptions. Hence, (3.47) is true.

Notice that

$$
\left(\Phi-\Phi_{\varepsilon_{k}}\right)(u)=-\varepsilon_{k} \int_{\Omega} G(u) d x \leq \varepsilon_{k} C(G, p)
$$


where the constant $C(G, p)$ does not depend on $u$, hence

$$
\Phi(u)<\Phi_{\varepsilon_{k}}(u)+\min \left\{1,-\sup _{K} \Phi_{\varepsilon_{k}}\right\} \quad \text { for every } u \in E
$$

if $k$ is large enough.

Fix $k$ such that (3.49) and (3.47) are true and set $\varepsilon:=\varepsilon_{k}$. Let $D$ be a large positive constant to be specified later, $D>-\inf _{K} \Phi_{\varepsilon}$. Choose $u_{0} \in K$. If the solution $u_{\varepsilon}(t):=u_{\varepsilon}\left(t ; u_{0}\right)$ blows up in a finite time $T$, then $\Phi_{\varepsilon}\left(u_{\varepsilon}(t)\right) \rightarrow-\infty$ as $t \rightarrow T$ - due to Theorem 3.8. If this solution exists globally, then its unboundedness implies $\Phi_{\varepsilon}\left(u_{\varepsilon}(t)\right) \rightarrow-\infty$ as $t \rightarrow \infty$ due to Theorems 3.7 and 3.2. Hence, in any case there exists $t_{\varepsilon}\left(u_{0}\right)=t_{\varepsilon}\left(u_{0}, D\right) \in(0, \infty)$ such that

$$
\Phi_{\varepsilon}\left(u_{\varepsilon}\left(t_{\varepsilon}\left(u_{0}\right)\right)\right)=-D .
$$

Since $t \mapsto \Phi_{\varepsilon}\left(u_{\varepsilon}(t)\right)$ is strictly decreasing, the mapping $t_{\varepsilon}: K \rightarrow(0, \infty): u_{0} \mapsto$ $t_{\varepsilon}\left(u_{0}\right)$ is continuous. Notice also that (3.49) implies

$$
\Phi\left(u_{\varepsilon}(t)\right)<\Phi_{\varepsilon}\left(u_{\varepsilon}(t)\right)-\sup _{K} \Phi_{\varepsilon} \leq 0 .
$$

Set $T:=\max _{K} t_{\varepsilon}$ and define the continuous map

$$
\eta:[0,1 / 2] \times K \rightarrow \Phi^{0} \backslash\{0\}:\left(s, u_{0}\right) \mapsto \begin{cases}u_{\varepsilon}\left(2 T s ; u_{0}\right) & \text { if } 2 T s \leq t_{\varepsilon}\left(u_{0}\right), \\ u_{\varepsilon}\left(t_{\varepsilon}\left(u_{0}\right) ; u_{0}\right) & \text { if } 2 T s>t_{\varepsilon}\left(u_{0}\right) .\end{cases}
$$

Since $K$ is bounded in $E$, it is easy to find $\tau>0$ small $\left(\tau<\min _{K} t_{\varepsilon}\right)$ such that the set $\left\{u_{\varepsilon}\left(\tau ; u_{0}\right): u_{0} \in K\right\}$ is bounded in $C(\bar{\Omega})$. Now Lemma 3.10 (used with $a$ and $\varepsilon$ replaced by $a-\varepsilon$ and $\varepsilon / 2$, respectively) guarantees that there exists $C_{1}>0$ such that $\left|u_{\varepsilon}\left(t ; u_{0}\right)\right| \leq C_{1}$ in $\Omega_{\varepsilon / 2}^{-}$for every $t \in\left[\tau, t_{\varepsilon}\left(u_{0}\right)\right]$ and every $u_{0} \in K$. Consequently, $\left|\eta\left(s, u_{0}\right)\right| \leq C_{1}$ in $\Omega_{\varepsilon / 2}^{-}$for every $s \in[\tau /(2 T), 1 / 2]$ and standard parabolic regularity estimates imply

$$
\left\|\eta\left(1 / 2, u_{0}\right)\right\|_{C^{1}\left(\overline{\Omega_{\varepsilon / 4}^{-}}\right)} \leq C_{2} .
$$

Notice that $\Phi_{\varepsilon}\left(\eta\left(1 / 2, u_{0}\right)\right)=-D$ and $\Phi \leq \Phi_{\varepsilon}+1$ imply

$$
\Phi\left(\eta\left(1 / 2, u_{0}\right)\right) \leq-D+1 \text {. }
$$

Let $\psi: \Omega \rightarrow \mathbb{R}$ be a smooth cut-off function, $\operatorname{supp} \psi \subset \Omega^{+}, \psi \equiv 1$ on $\Omega_{\varepsilon / 5}^{+}$. Then

$$
\eta\left(s, u_{0}\right):=2(1-s) \eta\left(1 / 2, u_{0}\right)+(2 s-1) \psi \eta\left(1 / 2, u_{0}\right), \quad s \in(1 / 2,1],
$$

is a continuous map, $\eta\left(1, u_{0}\right) \in H_{0}^{1}\left(\Omega^{+}\right)$, and using (3.50) we obtain

$$
\Phi\left(\eta\left(s, u_{0}\right)\right) \leq \Phi\left(\eta\left(1 / 2, u_{0}\right)\right)+C\left(C_{2}, \psi\right) \leq-D+1+C\left(C_{2}, \psi\right)<-M
$$

for every $s \in(1 / 2,1]$, provided $D$ is sufficiently large. This concludes the proof.

In 42, the existence of nontrivial equilibria of (3.1) is proved for $f(x, u)=$ $\lambda u+a(x) g(u)$ under some assumptions on $a, g$ which are different from those in (A1) and (A3). In particular, those assumptions allow the difference between $g(u)$ and $|u|^{p-1} u$ to grow faster than linearly so that the superlinearity assumption (3.5) need not be true. We will show that Theorems 3.2, 3.7, 3.8, Lemma 3.11 and Proposition 3.12 remain true (with minor modifications) if we replace (A3) with (A8). Notice that this new assumption enables superlinear perturbations of the power function $|u|^{p-1} u$. In fact the condition $g(u) /\left(|u|^{p-1} u\right) \rightarrow C$ as $|u| \rightarrow \infty$ in (A8) is even weaker than the corresponding hypothesis [42, (H5)]. Note also that 
the requirement $a \neq 0$ on $\partial \Omega$ in (A8) can be weakened to similar conditions as in [42, (H1)-(H2)]. On the other hand, we have to assume that $p<p_{2}^{*}$, where

$$
p_{2}^{*}:= \begin{cases}\frac{N+3}{N-1} & \text { if } N \leq 3, \\ \frac{8}{3} & \text { if } N=4, \\ p^{*} & \text { if } N>4,\end{cases}
$$

and $p^{*}$ is defined in (3.2).

Theorem 3.13. Assume (3.32), (A1), (A2) and (A8),

(a) Given $\eta>0$ there exists $C=C(\eta)$ such that (3.11) is true for all solutions $u$ satisfying (3.6) and (3.7).

(b) Let $u$ be a solution of (3.1) which blows up in a finite time $T$ in the $H^{1}(\Omega)$ norm. Then $\Phi(u(t)) \rightarrow-\infty$ as $t \rightarrow T-$.

(c) Let $K \subset E$ be compact and assume (3.45). Choose $M \geq 0$. Then there is a continuous map $\eta:[0,1] \times K \rightarrow \Phi^{0} \backslash\{0\}$ such that (3.46) is true.

Proof. (a) First let us show that (3.6) and (3.7) imply (3.8) (cf. Theorem 3.7). In fact, arguing by contradiction, as in the proof of Theorem 3.7 we obtain $s_{k}>0$ and solutions $u_{k}(x)=u_{k}\left(x, s_{k}\right)$ of the problems

$$
\begin{aligned}
-\Delta u_{k} & =a(x) g\left(u_{k}\right)+h_{k}(x), & & x \in \Omega, \\
u_{k} & =0, & & x \in \partial \Omega,
\end{aligned}
$$

where $h_{k}(x):=\lambda u_{k}\left(x, s_{k}\right)+h\left(x, u_{k}\left(x, s_{k}\right)\right)-\partial_{t} u_{k}\left(x, s_{k}\right)$ satisfy

$$
\int_{\Omega} h_{k}^{2} d x \leq C\left(1+\int_{\Omega} u_{k}^{2} d x\right)
$$

and the sequence $\left(u_{k}\right)$ is unbounded in $L^{2}(\Omega)$. Now one can repeat all estimates in [42, Section 2] (with $g_{k}:=g$ and with an obvious estimate of the additional term $h_{k}$ ) in order to obtain a contradiction. Consequently, (3.8) is true.

Next we prove the estimate

$$
\int_{t}^{t+\eta} \int_{\Omega}|\nabla u|^{2} d x d t \leq C_{1} \quad \text { for every } t \geq 0 .
$$

Assume on the contrary that

$$
\int_{t_{k}}^{t_{k}+\eta} \int_{\Omega}\left|\nabla u_{k}\right|^{2} d x d t \rightarrow \infty
$$

where $u_{k}(x, t)=u\left(x, t ; u_{0, k}\right)$ are global solutions satisfying $\left|\Phi\left(u_{0, k}\right)\right| \leq C$ and $\Phi\left(u_{k}(\cdot, t)\right) \geq-C$. Notice that (3.8) and (3.10) (with $u$ replaced by $u_{k}$ ) are true. Repeating the estimates in [42, Section 2] (with all quantities integrated over the time intervals $\left(t_{k}, t_{k}+\eta\right)$ ) we obtain a contradiction. Consequently, (3.52) is true.

Now we will proceed as in the proof of Theorem 3.2, starting with (3.14) replaced by (3.52). As in that proof, we may assume that $\left\|u_{0}\right\|_{1,2} \leq C$. Bounds (3.52) and (3.10) imply a uniform bound for $u(t)$ in $L^{r}(\Omega)$ whenever $r<2 N /(N-1)$, hence a bound in $H^{1}(\Omega)$ if $p<(N+3) /(N-1)$. If $p \geq(N+3) /(N-1)$ (and $\left.N>3\right)$, then we can repeat word by word the bootstrap argument in the proof of Theorem 3.2 . 
(b) Let $u$ blow up at time $T<\infty$. Assume on the contrary that $\Phi(u(t)) \geq-D$ for every $t<T$. As in the proof of Theorem 3.8 we obtain (3.40) and (3.41). Fix $\eta>0$ and assume that

$$
\int_{t_{k}}^{t_{k}+\eta} \int_{\Omega}|\nabla u|^{2} d x d t \rightarrow \infty \quad \text { as } k \rightarrow \infty
$$

where $t_{k}<T-\eta$. Then we obtain a contradiction in the same way as in the proof of (a). Hence (3.52) is true for $t \leq T-\eta$ and the same arguments as in (a) guarantee a uniform bound for $\|u(t)\|_{1,2}, t<T$, which yields a contradiction.

(c) We will mimic the proof of Proposition 3.12. In fact, in that proof we just have to notice that Lemma 3.11 remains true due to the estimates in [42, Section 2] (notice that the estimate (3.42) need not be true now).

Proposition 3.14. Assume (3.32), (A1), (A2), (A4), $a \in C(\bar{\Omega})$ and let the estimate in (A3) be true for some $p \in\left(1, p_{\mathrm{S}}\right)$. Fix $\varepsilon>0$. Then, given $\eta>0$, there exists $C=C(\eta, \varepsilon)$ such that

$$
\left\|\left.u(t)\right|_{\Omega_{\varepsilon}^{+}}\right\|_{\infty} \leq C \quad \text { for all } t>2 \eta,
$$

for all solutions u satisfying (3.6) and (3.7).

Proof. The proof is an easy modification of the local estimates in 24. In fact, choose a finite sequence $0<\varepsilon_{1}<\varepsilon_{2}<\cdots<\varepsilon_{k+1}<\varepsilon$, where $k$ is the number of steps in the bootstrap procedure in 24] (this number depends only on $p$ and $N)$. Set $\Omega_{i}:=\Omega_{\varepsilon_{i}}^{+}, i=1,2, \ldots, k+1$, and choose smooth cut-off functions $\psi_{i}$ with support in $\Omega_{i}$ such that $\psi_{i} \equiv 1$ in $\Omega_{i+1}, i=1,2, \ldots, k$. Define the local energies

$$
\begin{aligned}
\Phi_{i}(u) & :=\frac{1}{2} \int_{\Omega}\left(\left|\nabla\left(\psi_{i} u\right)\right|^{2}-\left|\nabla \psi_{i}\right|^{2} u^{2}\right) d x-\int_{\Omega} \psi_{i}^{2} F(x, u) d x, \\
\tilde{\Phi}_{i}(u) & :=\frac{1}{2} \int_{\Omega} \psi_{i}^{2}|\nabla u|^{2} d x-\int_{\Omega} \psi_{i}^{2} F(x, u) d x .
\end{aligned}
$$

As in 24] one can prove that $\Phi(u(t)), \tilde{\Phi}_{i}(u(t))$ are uniformly bounded for $t>\eta$. Then one can use the same arguments as in [24] (cf. also [39] or [40]) in order to show that the estimate

$$
\int_{s}^{s+1}\left\|\left.u(t)\right|_{\Omega_{i}}\right\|_{H^{1}\left(\Omega_{i}\right)}^{2 q} d t \leq C_{q} \quad \text { for all } s>\eta
$$

guarantees

$$
\int_{s}^{s+1}\left\|\left.u(t)\right|_{\Omega_{i+1}}\right\|_{H^{1}\left(\Omega_{i+1}\right)}^{2 \tilde{q}} d t \leq C_{\tilde{q}} \quad \text { for all } s>\eta,
$$

where $\tilde{q}<q+\frac{2}{p+1}$. Since (3.54) is true for $i=1$ and $q=2$ (see (3.14) in the proof of Theorem 3.2), we obtain (3.54) with $i=k$ and $q$ large. More precisely, $q$ is large enough to guarantee that interpolation between that estimate and

$$
\int_{s}^{s+1}\left\|\left.u_{t}(t)\right|_{\Omega_{k}}\right\|_{L^{2}\left(\Omega_{k}\right)}^{2} \leq C
$$

implies a uniform bound for $\left.u(t)\right|_{\Omega_{k}}$ in $L^{p+1}\left(\Omega_{k}\right)$. Now the boundedness of the energy $\tilde{\Phi}_{k}(u(t))$ implies a bound for $\left.u(t)\right|_{\Omega_{k+1}}$ in $H^{1}\left(\Omega_{k+1}\right)$. Finally, standard interior parabolic estimates guarantee (3.53). 
Corollary 3.15. Assume (3.32), (A1), (A2), (A4), $a \in C(\bar{\Omega})$ and let the estimate in (A3) be true for some $p \in\left(1, p_{\mathrm{S}}\right)$. Let $\Omega:=B_{R}(0)$ be the ball with center in the origin and radius $R$. Let $a$ and $h(\cdot, u)$ be radially symmetric, $a(0) \neq 0$.

(a) Given $\eta>0$ there exists $C=C(\eta)$ such that (3.11) is true for all radial solutions $u$ satisfying (3.6) and (3.7).

(b) Let $u$ be a radial solution of (3.1) which blows up in a finite time $T$ in the $H^{1}(\Omega)$-norm. Then $\Phi(u(t)) \rightarrow-\infty$ as $t \rightarrow T-$.

(c) Assume (3.44). Let $K \subset E$ be compact, consist of radial functions and assume (3.45). Choose $M \geq 0$. Then there is a continuous map $\eta:[0,1] \times$ $K \rightarrow \Phi^{0} \backslash\{0\}$ such that (3.46) is true and $\eta(t, u)$ is radial for all $(t, u) \in$ $[0,1] \times K$.

Proof. It is sufficient to prove assertion (a) since the remaining assertions can be obtained by a straightforward modification of the proofs of Theorem 3.8 and Proposition 3.12 .

If $a(0)<0$, then we obtain a uniform $L^{\infty}$ bound for $u(t), t>\eta$, in a small ball $B_{\varepsilon}(0)$ from Lemma 3.10 . If $a(0)>0$, then the same bound follows from Proposition 3.14. Proceeding as in the proof of Theorem 3.2 and using the fact that $u(x, t)=U(|x|, t)$, estimates (3.14) and (3.10) imply

$$
\int_{t}^{t+\eta}\left(\int_{\varepsilon}^{R} U_{r}^{2} d r\right)^{2} d t+\int_{t}^{t+\eta} \int_{\varepsilon}^{R} U_{t}^{2} d r d t \leq C
$$

and interpolation yields a uniform $L^{\infty}$ bound for $U(t)$ in $B_{R}(0) \backslash B_{\varepsilon}(0)$ (see [40]). Consequently, $u(t)$ is bounded in $L^{\infty}(\Omega)$. Now the assertion is obvious.

In the following theorem we show that the exponent $p_{1}^{*}$ in Theorem 3.2 can be increased if $N=2$, provided $f$ has the form (3.32) and $a$ satisfies some additional assumptions. In the subsequent remark we indicate how one can use that result in order to prove the existence of nodal equilibria of (3.1) even if $a$ does not satisfy those additional assumptions.

Theorem 3.16. Let $N=2$. Assume (3.32), (A1), (A2), (A4), $a \in C(\bar{\Omega})$ and let the estimate in (A3) be true for some $p \in(1,5+4 \sqrt{2})$. Assume that $\operatorname{dist}\left(\Omega^{+}, \Omega^{-}\right)>$ 0 and $a_{+}^{1 /(p-1)} \in W^{2, \infty}(\Omega)$. Then, given $\eta>0$, there exists $C=C(\eta)$ such that (3.11) is true for all solutions u satisfying (3.6) and (3.7).

Proof. Let $u$ satisfy (3.6) and (3.7). The estimates in Theorems [3.2, 3.6 and 3.7 guarantee a uniform bound for $u(t)$ in $L^{2}(\Omega)$ and for $\nabla u$ in $L^{4}\left((t, t+\eta), L^{2}(\Omega)\right)$, $t>\eta$.

Denote $g_{1}(u):=g(u)-|u|^{p-1} u, b(x):=a_{+}(x)^{1 /(p-1)}$ and $v:=b u$. Multiplying the equation in (3.1) by $b$ we see that $v$ solves the problem

$$
\left\{\begin{aligned}
v_{t}-\Delta v & =|v|^{p-1} v+w, & & x \in \Omega, t>0, \\
v & =0, & & x \in \partial \Omega, t>0, \\
v(x, 0) & =b(x) u_{0}(x), & & x \in \Omega,
\end{aligned}\right.
$$

where $w:=b^{p} g_{1}(u)+b h(\cdot, u)-\Delta b u-2 \nabla b \cdot \nabla u$ is bounded in $L^{4}\left((t, t+\eta), L^{2}(\Omega)\right)$ for $t>\eta$. Using the estimates in [6, Theorem 4.1] we get a uniform bound for $v(t)$ in $L^{\infty}(\Omega)$ for all $t>2 \eta$ (notice that the hypotheses of that theorem require $p<5+4 \sqrt{2})$. 
Set $\Omega_{1}:=\operatorname{int}\left(\Omega^{+} \cup \Omega^{0}\right)$. Then $u$ solves the problem

$$
\left\{\begin{aligned}
u_{t}-\Delta u & =|v|^{p-1} u+w_{1}, & & x \in \Omega_{1}, t>0, \\
u & =0, & & x \in \partial \Omega \cap \partial \Omega_{1}, t>0,
\end{aligned}\right.
$$

where $\left.v(t)\right|_{\Omega_{1}}$ is bounded in $L^{\infty}\left(\Omega_{1}\right)$ for $t>2 \eta$ and $\left.u(t)\right|_{\Omega_{1}}, w_{1}(t)$ are bounded in $L^{2}\left(\Omega_{1}\right)$ for $t>\eta$. Since $N=2$, parabolic estimates guarantee an $L^{\infty}$ bound for $\left.u(t)\right|_{\Omega^{+}}$for all $t>3 \eta$. Here we use the fact that $\operatorname{dist}\left(\Omega^{+}, \Omega^{-}\right)>0$.

Set $\Omega_{2}:=\operatorname{int}\left(\Omega^{-} \cup \Omega^{0}\right)$. Then $u$ solves the problem

$$
\left\{\begin{aligned}
u_{t}-\Delta u & =a(x)|u|^{p-1} u+w_{2}, & & x \in \Omega_{2}, t>0, \\
u & =0, & & x \in \partial \Omega \cap \partial \Omega_{2}, t>0,
\end{aligned}\right.
$$

where $a(x) \leq 0$ for $x \in \Omega_{2},\left.u(t)\right|_{\Omega_{2}}, w_{2}(t)$ are bounded in $L^{2}\left(\Omega_{2}\right)$ for $t>\eta$ and $u(x, t)$ is bounded for $x \in \partial \Omega_{2} \backslash \partial \Omega$ and $t>3 \eta$ due to the estimate of $\left.u(t)\right|_{\Omega^{+}}$. The maximum principle and parabolic estimates yield an $L^{\infty}$ bound for $\left.u(t)\right|_{\Omega_{2}}$ for all $t>4 \eta$. Consequently, $u(t)$ is bounded in $L^{\infty}(\Omega)$ for $t>4 \eta$. Finally, the estimate (3.11) (for $t>5 \eta$ ) follows by standard arguments.

Remark 3.17. Let, for example, $a$ satisfy (A6), $a_{k}, k=1,2, \ldots$, satisfy the assumptions of Theorem 3.16 and $a_{k} \rightarrow a$ in $C(\bar{\Omega})$. Assume for simplicity that $\lambda<\lambda_{1}$. Then one can use the arguments in Section $\left[5\right.$ in order to find nodal equilibria $w_{k}$ (corresponding to $a_{k}$ ) such that $w_{k}$ connects to zero, $k=1,2, \ldots$ In addition, the corresponding energies $\Phi_{k}\left(w_{k}\right)$ remain bounded since we can find the equilibria $w_{k}$ in the $\omega$-limit sets of initial data $u_{0, k}$ which are uniformly bounded in $H^{1}(\Omega)$, hence

$$
C \geq \Phi_{k}\left(u_{0, k}\right) \geq \Phi_{k}\left(w_{k}\right) \geq \Phi_{k}(0)=0 .
$$

Now similarly as in the proof of Lemma 3.11 (cf. also [42]) we obtain the boundedness of $\left(w_{k}\right)$, hence one can pass to the limit in order to see that $w:=\lim _{k} w_{k}$ is a nodal equilibrium of the limiting problem (to prove that $w$ is nodal it is sufficient to use the fact that the zero solution is an isolated equilibrium for every subdomain of $\Omega$ ). However, the existence of a connection from $w$ to the zero solution does not seem to be clear.

\section{Signed Equilibria}

This section is devoted to the existence of signed equilibria.

Let us start by stating some facts about the general equation (3.1), assuming only $f \in \mathcal{F}_{\mathrm{S}}$. Also consider the related stationary problem

$$
\left\{\begin{aligned}
-\Delta u & =f(x, u), & & x \in \Omega, \\
u & =0, & & x \in \partial \Omega .
\end{aligned}\right.
$$

A supersolution (subsolution) $u$ of (4.1) is, by definition, a function in $u \in W^{2,1}(\Omega) \cap$ $C^{1}(\bar{\Omega})$ satisfying $-\Delta u \geq f(x, u)(-\Delta u \leq f(x, u))$ for a.e. $x \in \Omega$ and $u(x) \geq 0$ $(u(x) \leq 0)$ for $x \in \partial \Omega$. A strict supersolution (subsolution) of (4.1) is a supersolution (subsolution) of (4.1) that is not a solution of (4.1). The comparison principle yields that the solution $u(t)$ of (3.1) satisfies $u(t) \leq \bar{u}$ whenever $u_{0} \leq \bar{u}$ and $\bar{u}$ is a supersolution of (4.1) (an analogous assertion is true for the subsolution). Moreover, the following lemma can be proved by standard methods. 
Lemma 4.1. Let $f \in \mathcal{F}_{\mathrm{S}}$. Suppose that $\bar{u}$ is a strict supersolution of (4.1), that $\underline{u}$ is a strict subsolution of (4.1), and that $\underline{u} \leq \bar{u}$. Let

$$
[\underline{u}, \bar{u}]:=\{u \in E: \underline{u} \leq u \leq \bar{u}\}
$$

denote the order interval in $E$. Then the following hold:

(a) Every semiorbit starting in $[\underline{u}, \bar{u}]$ is a global semiorbit.

(b) For all $t>0$ the set $\mathcal{O}_{+}\left(\varphi^{t}([\underline{u}, \bar{u}])\right)$ is relatively compact.

(c) $\Phi$ is bounded below on $[\underline{u}, \bar{u}]$ and attains its minimum at an equilibrium in $[\underline{u}, \bar{u}]$.

(d) The interval $[\underline{u}, \bar{u}]$ is strictly positive invariant in the following sense: For all $u \in[\underline{u}, \bar{u}]$ and $t>0$ it holds that $\varphi^{t}(u) \in \operatorname{int}_{X}([\underline{u}, \bar{u}] \cap X)$.

(e) For all $t>0$ there is an open neighborhood $U$ of $[\underline{u}, \bar{u}]$ in $E$ such that $\varphi^{t}(u) \in[\underline{u}, \bar{u}]$ for all $u \in U$.

(f) The set $\mathcal{A}([\underline{u}, \bar{u}])$ is open.

In what follows, for an open subset $V$ of $\mathbb{R}^{N}$ denote by $\lambda_{1}(V)$ the first eigenvalue of $-\Delta$ in $H_{0}^{1}(V)$, and by $e_{1}(V)$ the corresponding positive eigenfunction, normalized by $\sup _{V} e_{1}(V)=1$. We now specialize to nonlinearities $f$ having the structure

$$
f(x, u)=\lambda u+a(x) g(u)+h(x, u) .
$$

Lemma 4.2. Assume (A1) and (A5), For every $\lambda>\lambda_{1}$ there is $\varepsilon_{0}>0$ such that $\varepsilon e_{1}(\Omega)$ is a strict subsolution and $-\varepsilon e_{1}(\Omega)$ is a strict supersolution of $\left(E_{\lambda}\right)$ for all $\varepsilon$ in $\left(0, \varepsilon_{0}\right]$.

Proof. As (A1) and (A5) are satisfied, there exists $\delta>0$ such that

$$
\forall x \in \Omega, \forall|u|<\delta:\left(\lambda-\lambda_{1}\right)|u| \geq|a(x)||g(u)|+|h(x, u)| .
$$

It is enough to choose $\varepsilon_{0}<\delta$.

Lemma 4.3. Suppose that (A1), (A2) and (A5) hold, and $g$ fulfills either (A3)] or the condition in $(\mathrm{A} 8)$, Then the sets $\Lambda^{+}$and $\Lambda^{-}$are bounded above.

Proof. We prove the lemma only for $\Lambda^{+}$, the proof for $\Lambda^{-}$being similar. As $\Omega^{+}$is a nonempty open set, there exist $x \in \Omega^{+}$and $\rho>0$ such that $U:=\{y:|y-x|<$ $\rho\} \subset \Omega^{+}$.

Let $\lambda \in \Lambda^{+}$and $u$ be the corresponding positive solution of $\left(E_{\lambda}\right)$. Testing $\left(E_{\lambda}\right)$ with $e_{1}(U)$ we obtain

$$
\begin{aligned}
\int_{U}\left(\lambda u e_{1}(U)\right. & \left.+a g(u) e_{1}(U)+h(x, u) e_{1}(U)\right) d x=\int_{U} \nabla u \nabla e_{1}(U) d x \\
& =-\int_{U} u \Delta e_{1}(U) d x+\int_{\partial U} u \frac{\partial e_{1}(U)}{\partial \nu} d S .
\end{aligned}
$$

As $u>0$ and $\partial e_{1}(U) / \partial \nu<0$ on $\partial U$ we get

$$
\left(\lambda-\lambda_{1}(U)\right) \int_{U} u e_{1}(U) d x \leq-\int_{U}\left(a g(u) e_{1}(U)+h(x, u) e_{1}(U)\right) d x .
$$

Using the fact that $U \subset \Omega^{+}$, (A5) and the assumptions on the growth of $h$ and $g$, we get the existence of $M>0$ satisfying $-a(x) g(w)-h(x, w)<M w$ for all $w \geq 0$ and $x \in U$. From $e_{1}(U)>0$ and $\int_{U} u e_{1}(U) d x>0$ we obtain $\lambda<M+\lambda_{1}(U)$. 
Remark 4.4. We will prove in Section 5 that (under suitable assumptions on $a, g$ and h) $\left(-\infty, \lambda^{+}\right) \subset \Lambda^{+}$and $\Lambda^{+}=\left(-\infty, \lambda^{+}\right]$if $\lambda^{+}>\lambda_{1}$. Of course, similar assertions are true for the set $\Lambda^{-}$.

Remark 4.5. If $h \equiv 0, q>1, g(u) / u^{q} \rightarrow C>0$ as $u \rightarrow 0+$ and

$$
\int_{\Omega} a(x) e_{1}^{q+1}(\Omega) d x<0
$$

then a local bifurcation analysis at $\lambda=\lambda_{1}$ shows that $\lambda^{+}>\lambda_{1}$; see the proof of 3 , Lemma 2.3], for example. (If $a$ is not Hölder continuous, then one has to replace the spaces $C_{0}^{2, \beta}$ and $C^{0, \beta}$ in that proof with $W^{2, r} \cap W_{0}^{1, r}$ and $L^{r}$ respectively, where $r>1$ is large enough.) In some cases, the condition (4.2) is also necessary. More precisely, if $g(u)=|u|^{q-1} u$ and $\lambda_{1} \in \Lambda^{+}$, then the condition (4.2) has to be satisfied; see [3, Lemma 1.3]. Again, similar assertions are true for the set $\Lambda^{-}$.

Our next aim is to prove that $\lambda^{ \pm}$may be arbitrarily large.

Proposition 4.6. Assume that $g \in C^{1}(\mathbb{R}, \mathbb{R}), g \in \mathcal{F}_{\mathrm{S}}, g^{\prime}(0)=0, g>0$ for $u>0$ small, $h=0$ in $\Omega \times \mathbb{R}$, and $\lambda>\lambda_{1}$. Then there exists $a \in C^{\infty}(\bar{\Omega})$ such that $\lambda^{+}=\lambda^{+}(a, g, h)>\lambda$ and such that $\Omega^{+}$and $\Omega^{-}$are nonempty open sets.

The function a can be constructed such that 0 is a regular value of $a$ and $a \neq 0$ on $\partial \Omega$. On the other hand, we can also achieve that $\operatorname{dist}\left(\Omega^{+}, \Omega^{-}\right)>0$ and condition (A4.2) from (A4) is satisfied.

Proof. First we construct $a \in C^{\infty}(\bar{\Omega})$ with the required properties such that $\left(E_{\lambda}\right)$ possesses a positive strict supersolution $u \in C^{\infty}(\bar{\Omega})$. More precisely, we construct $a$ and $u$ such that

$$
\begin{array}{rlrl}
-\Delta u & >\lambda u+a g(u) & & \text { in } \Omega, \\
u>0 & & \text { in } \bar{\Omega} .
\end{array}
$$

Let $x_{0} \in \Omega$ and $B_{\varepsilon}\left(x_{0}\right):=\left\{x:\left|x-x_{0}\right|<\varepsilon\right\}$. There exists $\varepsilon_{0} \in\left(0, \operatorname{dist}\left(x_{0}, \partial \Omega\right)\right)$ such that $\lambda_{1}\left(B_{\varepsilon}\left(x_{0}\right)\right)>2 \lambda$ for all $\varepsilon \in\left(0, \varepsilon_{0}\right]$. We define $\Omega_{1}:=B_{\varepsilon_{0}}\left(x_{0}\right), \Omega_{2}:=$ $B_{\varepsilon_{0} / 2}\left(x_{0}\right), \Omega_{3}:=B_{\varepsilon_{0} / 3}\left(x_{0}\right)$ and functions $\eta_{i}: \bar{\Omega} \rightarrow[0,1], i=1,2$, such that $\eta_{i} \in$ $C^{\infty}(\bar{\Omega})$ and

$$
\eta_{i}(x) \begin{cases}=0, & x \in \overline{\Omega \backslash \Omega_{i}}, \\ =1, & x \in \overline{\Omega_{i+1}}, \\ \in(0,1) & \text { else. }\end{cases}
$$

Let $K>0$ be such that $g(t)>0$ for $t \in(0,2 K)$. Set

$$
u=K\left(e_{1}\left(\Omega_{1}\right) \eta_{1}+1-\eta_{1}\right) \text { in } \bar{\Omega} .
$$

Obviously, $u \in C^{\infty}(\bar{\Omega}), K \geq u>0$ in $\bar{\Omega}$ and

$$
-\Delta u-\lambda u \begin{cases}=-K \lambda & \text { in } \overline{\Omega \backslash \Omega_{1}}, \\ >K \lambda e_{1}\left(\Omega_{1}\right) & \text { in } \overline{\Omega_{2}} .\end{cases}
$$

In order to satisfy (4.3) it is enough to choose $a \in C^{\infty}(\bar{\Omega})$,

$$
a<\frac{-\Delta u-\lambda u}{g(u)} \text { in } \bar{\Omega}
$$

as $g(u)>0$ in $\bar{\Omega}$. 
Due to (4.5) we obtain

$$
\alpha_{1}:=\min _{\bar{\Omega}_{2}} \frac{-\Delta u-\lambda u}{g(u)}>0, \quad \alpha_{2}:=\min _{\bar{\Omega}} \frac{-\Delta u-\lambda u}{g(u)}<0 .
$$

Defining $a:=\alpha_{2}+\left(\alpha_{1}-\alpha_{2}\right) \eta_{2}-\alpha_{1} / 2$, we have $a \in C^{\infty}(\bar{\Omega}), a \neq 0$ on $\partial \Omega, a>0$ on $\Omega_{3}$ and $a<0$ on $\Omega \backslash \overline{\Omega_{2}}$. It is clear that it is possible to choose $\eta_{2}$ such that 0 is a regular value of $a$ or that (A4.2) from (A4) holds.

Observe that for $\varepsilon>0$ small enough the function $\varepsilon e_{1}(\Omega)$ is a positive strict subsolution of $\left(E_{\lambda}\right)$ by Lemma 4.2, and $\varepsilon e_{1}(\Omega) \leq u$ due to (4.4). Now the claim follows applying Lemma 4.1](c) to $\left[\varepsilon e_{1}(\Omega), u\right]$.

\section{Equilibria AND CONNECTING ORBITS FROM A PRIORI ESTIMATES}

To have the a priori estimates of Section 3 at our disposal, throughout this section we consider $\left(P_{\lambda}\right)$ and assume (A1), (A2) and either (A8) or (A3) and (A4), In addition, we assume (A5), As before, $\varphi$ denotes the semiflow induced by $\left(P_{\lambda}\right)$ on $E$.

For convenience denote

$$
\|u\|=\left(\int_{\Omega}|\nabla u|^{2} d x\right)^{1 / 2}
$$

and define $\Psi: E \rightarrow \mathbb{R}$ by

so

$$
\Psi(u)=\int_{\Omega}(a(x) G(u)+H(x, u)) d x
$$

Moreover put

$$
\Phi(u)=\frac{1}{2}\left(\|u\|^{2}-\lambda\|u\|_{2}^{2}\right)-\Psi(u) .
$$

$$
\mathcal{K}:=\left\{u \in E \backslash\{0\}: \Phi^{\prime}(u)=0\right\},
$$

the set of nontrivial critical points of $\Phi$.

If $c \in \mathbb{R}$ call $\left(u_{n}\right) \subset E$ a $(\mathrm{PS})_{c}$-sequence for $\Phi$ if $\Phi\left(u_{n}\right) \rightarrow c$ and $\Phi^{\prime}\left(u_{n}\right) \rightarrow 0$ as $n \rightarrow \infty$. If every $(\mathrm{PS})_{c}$-sequence is relatively compact we say that $\Phi$ satisfies the $(\mathrm{PS})_{c}$-condition. If $\Phi$ satisfies the $(\mathrm{PS})_{c}$-condition for every $c \in \mathbb{R}$, then $\Phi$ is said to satisfy the (PS)-condition.

Lemma 5.1. If $\left(u_{n}\right) \subset H_{0}^{1}\left(\Omega^{+}\right)$satisfies $\Phi\left(u_{n}\right) \leq C$ and $\left|\Phi^{\prime}\left(u_{n}\right) u_{n}\right| \leq C\left\|u_{n}\right\|$, then $\left\|u_{n}\right\|$ remains bounded.

Proof. Suppose that $\left(u_{n}\right) \subset H_{0}^{1}\left(\Omega^{+}\right)$satisfies $\left\|u_{n}\right\| \rightarrow \infty$. First of all $\Phi^{\prime}\left(u_{n}\right) u_{n}=$ $O\left(\left\|u_{n}\right\|\right)$ yields

$$
\Psi^{\prime}\left(u_{n}\right) u_{n}=\left\|u_{n}\right\|^{2}+O\left(\left\|u_{n}\right\|+\left\|u_{n}\right\|_{2}^{2}\right) .
$$

Since $g(u) u \geq \theta G(u)-C$ for some $\theta>2$ and $C>0$, we obtain

$$
\begin{aligned}
C \geq \Phi\left(u_{n}\right) & =\frac{1}{2}\left(\left\|u_{n}\right\|^{2}-\lambda\left\|u_{n}\right\|_{2}^{2}\right)-\Psi\left(u_{n}\right) \\
& \geq \frac{1}{2}\left(\left\|u_{n}\right\|^{2}-\lambda\left\|u_{n}\right\|_{2}^{2}\right)-\frac{1}{\theta} \Psi^{\prime}\left(u_{n}\right) u_{n}+O\left(\left\|u_{n}\right\|_{2}^{2}\right) .
\end{aligned}
$$

This estimate and (5.1) imply

$$
\left\|u_{n}\right\|^{2}=O\left(\left\|u_{n}\right\|_{2}^{2}\right) .
$$


Furthermore (5.1) yields

$$
\int_{\Omega^{+}} a(x)\left|u_{n}\right|^{p+1} d x=O\left(\left\|u_{n}\right\|^{2}\right) .
$$

Set $v_{n}=u_{n} /\left\|u_{n}\right\|$. We may assume that $v_{n} \rightarrow v$ in $H_{0}^{1}\left(\Omega^{+}\right)$, and hence that $v_{n} \rightarrow v$ in $L^{q}\left(\Omega^{+}\right)$for $q \in\left[2, p_{\mathrm{S}}+1\right)$. Now (5.3) implies that

$$
\int_{\Omega^{+}} a(x)|v|^{p+1} d x=0,
$$

which together with $a(x)>0$ on $\Omega^{+}$yields $v=0$. This in turn implies $v_{n} \rightarrow 0$ in $L^{2}(\Omega)$, in contradiction with (5.2).

Corollary 5.2. There is $C_{\min } \leq 0$ such that $\Phi(u) \geq C_{\min }$ for every $u \in H_{0}^{1}\left(\Omega^{+}\right)$ with $\Phi^{\prime}(u) u=0$.

Corollary 5.3. The restriction of $\Phi$ to a closed subspace of $H_{0}^{1}\left(\Omega^{+}\right)$satisfies the (PS)-condition.

Lemma 5.4. If $Y$ is a finite dimensional subspace of $H_{0}^{1}\left(\Omega^{+}\right)$and $u_{0} \in E$, then

$$
\lim _{\substack{\|u\| \rightarrow \infty \\ u \in Y}} \Phi\left(u+u_{0}\right)=-\infty .
$$

Proof. Consider the weighted norm

$$
\|u\|_{a}=\left(\int_{\Omega^{+}} a(x)|u|^{p+1} d x\right)^{\frac{1}{p+1}}
$$

on $H_{0}^{1}\left(\Omega^{+}\right)$. On $Y$ it is equivalent with $\|\cdot\|$. The claim now follows since

$$
\Phi\left(u+u_{0}\right) \leq-c\|u\|_{a}^{p+1}+O\left(\|u\|^{2}\right) \text { as }\|u\| \rightarrow \infty
$$

for $u \in H_{0}^{1}\left(\Omega^{+}\right)$.

In the sequel we apply some of the results from 11. Note that assumptions (F1), (F2), (F4)-(F6) in that paper follow from our general assumptions in this section and from the a priori estimates proved in Theorems 3.23 .7 and 3.8 Assumption (F3) in [1 is not satisfied in general in the present setting. Nevertheless, the facts proved in Lemmata 5.1 and 5.4 and in Corollaries 5.2 and 5.3 are sufficient for the application of the results from [1] as we will show. In addition, the a priori estimates from Section 3 and the compactness of $\varphi$ imply the following:

If $A \subset E$ is closed and inf $\Phi(A)>-\infty$, then every positive semiorbit starting in $\overline{\mathcal{A}(A)}$ exists for all positive times and is relatively compact.

Proposition 5.5. If $\lambda<\lambda_{1}$, then there are a positive equilibrium $u^{+}$and a negative equilibrium $u^{-}$such that $u^{ \pm} \succ 0$.

Proof. The set $\mathcal{A}(0)$ is an open neighborhood of 0 . Choose $v_{0} \in H_{0}^{1}\left(\Omega^{+}\right)$such that $v_{0}>0$ and denote by $V$ the span of $\left\{v_{0}\right\}$ in $E$. By Lemma $5.4 \mathcal{A}(0) \cap V$ is bounded. The rest of the proof is the same as that of [1, Thm. 3.1] if one replaces $E_{1}$ by $V$.

Proposition 5.6. If $\lambda<\lambda_{2}$, then there is a nodal equilibrium $u^{*}$ such that $u^{*} \succ 0$. 
Proof. Put

$$
W_{2}:=\left\{u \in E: T_{+}(u)=\infty, \limsup _{t \rightarrow \infty}\|\varphi(t, u)\|_{1,2}^{1 / t} \leq e^{\lambda-\lambda_{2}}\right\} .
$$

In the terminology of [1] this is the second superstable manifold of $\varphi$ at 0 . It is a $C^{1}$-submanifold of $E$ of codimension 1, tangent in 0 to the generalized eigenspace $Z$ of $L$ corresponding to the spectral set $\sigma(L) \cap\left(\lambda_{1}, \infty\right)$. Choose linearly independent $v_{1}, v_{2} \in H_{0}^{1}\left(\Omega^{+}\right)$such that $v_{1}>0$. Denote by $V_{1}$ the span of $\left\{v_{1}\right\}$ and by $V_{2}$ the span of $\left\{v_{1}, v_{2}\right\}$. Note that $E=V_{1} \oplus Z$. Denote by $P$ the projection in $E$ onto $Z$ with kernel $V_{1}$. It follows as in the proof of [1, Thm. 2.6] that the restriction of $P$ to $W_{2}$ is a diffeomorphism onto an open neighborhood of 0 in $Z$. By Lemma 5.4 the set $\Phi^{0} \cap V_{2}$ is bounded. Replacing $E_{3}^{-}$by $V_{2}$ and $E_{2}$ by $Z$ the proof can be finished following the arguments in the proof of [1, Thm. 3.2a)].

In the next existence result the local structure of the semiflow at 0 will play an important rôle. Therefore recall that for $\lambda \in \mathbb{R}$ there exist local (strong) stable and unstable $C^{1}$-manifolds $W_{\text {loc }}^{\mathrm{s}}$ and $W_{\text {loc }}^{\mathrm{u}}$ at 0 with respect to $\varphi$. They are given as $C^{1}$-graphs over neighborhoods of 0 in the (strong) stable and unstable generalized eigenspaces $E^{\mathrm{s}}$ and $E^{\mathrm{u}}$, the generalized eigenspaces of $L$ corresponding to the spectral sets $\sigma(L) \cap(\lambda, \infty)$ and $\sigma(L) \cap\left[\lambda_{1}, \lambda\right)$. Furthermore, they are contained in the global stable and unstable sets $W^{\mathrm{s}}$ and $W^{\mathrm{u}}$ of 0 :

$$
\begin{aligned}
W^{\mathrm{s}} & :=\{u \in E: \varphi(t, u) \rightarrow 0 \text { as } t \rightarrow \infty\}, \\
W^{\mathrm{u}}: & :=\{u \in E: \varphi(t, u) \rightarrow 0 \text { as } t \rightarrow-\infty\} .
\end{aligned}
$$

Proposition 5.7. Assume that (A6) holds.

(a) If $\lambda \in \mathbb{R} \backslash \sigma(L)$, then there is a nontrivial equilibrium $u^{*}$ such that either $u^{*} \succ 0$ or $0 \succ u^{*}$.

(b) If $\lambda \in \sigma(L)$ and if in addition (A7) holds, then there is a nontrivial equilibrium $u^{*}$ such that either $u^{*} \succ 0$ or $\Phi\left(u^{*}\right) \leq 0$.

The equilibrium $u^{*}$ obtained in (a) and (b) above is nodal in the case $u^{*} \succ 0$.

Proof. (a) Denote by $P^{\mathrm{u}}$ and $P^{\mathrm{s}}$ the projections in $E$ onto $E^{\mathrm{u}}$ and $E^{\mathrm{s}}$, respectively. If $u \in E$ we write $u^{\mathrm{u}}:=P^{\mathrm{u}} u$ and $u^{\mathrm{s}}:=P^{\mathrm{s}} u$. The local unstable and stable manifolds $W_{\mathrm{loc}}^{\mathrm{u}}$ and $W_{\mathrm{loc}}^{\mathrm{s}}$ are given as the graphs of maps $\xi_{\mathrm{u}} \in C^{1}\left(V^{\mathrm{u}}, E^{\mathrm{s}}\right)$ and $\xi_{\mathrm{s}} \in C^{1}\left(V^{\mathrm{s}}, E^{\mathrm{u}}\right)$, where $V^{\mathrm{u}} \subset E^{\mathrm{u}}$ and $V^{\mathrm{s}} \subset E^{\mathrm{s}}$ are open neighborhoods of 0 . Moreover, $\xi_{\mathrm{u}}(0)=0, \xi_{\mathrm{u}}^{\prime}(0)=0, \xi_{\mathrm{s}}(0)=0$, and $\xi_{\mathrm{s}}^{\prime}(0)=0$. Hence we may also assume that

$$
\sup _{v \in V^{\mathrm{u}}}\left\|\xi_{\mathrm{u}}^{\prime}(v)\right\| \leq \frac{1}{2} \quad \text { and } \quad \sup _{v \in V^{\mathrm{s}}}\left\|\xi_{\mathrm{s}}^{\prime}(v)\right\| \leq \frac{1}{2} .
$$

Set $\Xi_{\mathrm{u}}(v):=v+\xi_{\mathrm{u}}(v)$ for $v \in V^{\mathrm{u}}$ and $\Xi_{\mathrm{s}}(v):=v+\xi_{\mathrm{s}}(v)$ for $v \in V^{\mathrm{s}}$. The global unstable and stable sets of 0 are then given by $W^{\mathrm{u}}:=\mathcal{O}_{+}\left(W_{\text {loc }}^{\mathrm{u}}\right)$ and $W^{\mathrm{s}}:=$ $\mathcal{O}_{-}\left(W_{\text {loc }}^{\mathrm{s}}\right)$ respectively.

If $Y$ is a subspace of $E$, then we set

$U_{r} Y:=\{u \in Y:\|u\|<r\}, B_{r} Y:=\{u \in Y:\|u\| \leq r\}, S_{r} Y:=\{u \in Y:\|u\|=r\}$.

Choose $r>0$ such that

$$
B_{r} E^{\mathrm{u}} \subset V^{\mathrm{u}} \quad \text { and } \quad B_{r} E^{\mathrm{s}} \subset V^{\mathrm{s}} .
$$


Set

$$
\begin{array}{ll}
\mathcal{B}^{\mathrm{s}}:=\Xi_{\mathrm{s}}\left(B_{r} E^{\mathrm{s}}\right), & \mathcal{S}^{\mathrm{s}}:=\Xi_{\mathrm{s}}\left(S_{r} E^{\mathrm{s}}\right), \\
\mathcal{B}^{\mathrm{u}}:=\Xi_{\mathrm{u}}\left(B_{r} E^{\mathrm{u}}\right), & \mathcal{S}^{\mathrm{u}}:=\Xi_{\mathrm{u}}\left(S_{r} E^{\mathrm{u}}\right) .
\end{array}
$$

It was shown in [1, Lemma 2.1] that

$$
C_{\mathrm{s}}:=\inf \Phi\left(\mathcal{S}^{\mathrm{s}}\right)>0 \text {. }
$$

Put $A:=\Phi^{0} \backslash\{0\}$. Since $\Phi(u)>0$ for $u$ in $W^{\mathrm{s}} \backslash\{0\}$,

$$
\mathcal{B}^{\mathrm{s}} \cap A=\varnothing \text {. }
$$

Clearly $\Phi(u)<0$ for every $u$ in $\mathcal{S}^{\mathrm{u}}$. If there is no $u^{*} \in \mathcal{K}$ such that $0 \succ u^{*}$, then $\mathcal{O}_{+}(u)$ is unbounded for every $u \in \mathcal{S}^{u}$ (since every bounded positive semiorbit is global and relatively compact). By Proposition 3.12 there is $\eta_{1} \in C\left([0,1] \times \mathcal{S}^{\mathrm{u}}, E\right)$ such that

$$
\begin{aligned}
\eta_{1}(0, v) & =v, \\
K_{1}:=\eta_{1}\left(1, \mathcal{S}^{\mathrm{u}}\right) & \subset \Phi^{C_{\min }-2} \cap H_{0}^{1}\left(\Omega^{+}\right), \\
\eta_{1}\left([0,1] \times \mathcal{S}^{\mathrm{u}}\right) & \subset A .
\end{aligned}
$$

Since $K_{1}$ is compact and $\Phi^{\prime}$ is bounded on bounded subsets we may find an approximating finite dimensional subspace $Y$ of $H_{0}^{1}\left(\Omega^{+}\right)$with the following property: If we denote by $P_{Y}$ the orthogonal projection onto $Y$ and if we define $\eta_{2} \in C\left([0,1] \times K_{1}, H_{0}^{1}\left(\Omega^{+}\right)\right)$by $\eta_{2}(t, v):=(1-t) v+t P_{Y} v$, then

$$
\begin{aligned}
\eta_{2}(0, v) & =v, \\
K_{2}:=\eta_{2}\left(1, K_{1}\right) & \subset \Phi^{C_{\min }-1}, \\
\eta_{2}\left([0,1] \times K_{1}\right) & \subset A .
\end{aligned}
$$

Choose a finite dimensional subspace $Z$ of $H_{0}^{1}\left(\Omega^{+}\right)$, strictly containing $Y$. By Lemma 5.4 there is $R \geq 0$ such that $\Phi(u) \leq 0$ for $u$ in $Z \backslash B_{R} Z$. Denote $C_{R}:=$ $\min \Phi\left(B_{2 R} Y\right)$. By Corollary $\left.5.2 \Phi\right|_{Y}$ has no critical point in $Y \cap \Phi^{C_{\min }-1}$. Moreover, Corollary 5.3 ensures that $\left.\Phi\right|_{Y}$ satisfies the (PS)-condition. In view of (5.7) we can therefore find a deformation $\eta_{3} \in C\left([0,1] \times K_{2}, Y\right)$ such that

$$
\begin{aligned}
\eta_{3}(0, v) & =v, \\
K_{3}:=\eta_{3}\left(1, K_{2}\right) & \subset \Phi^{C_{R}-1} \cap Y \subset Y \backslash B_{2 R} Y, \\
\eta_{3}\left([0,1] \times K_{2}\right) & \subset A .
\end{aligned}
$$

We now fix $v_{0} \in S_{2 R} Z \cap Y^{\perp}$ and define $\eta_{4} \in C\left([0,1] \times K_{3}, Z\right)$ by $\eta_{4}(t, v):=$ $(1-t) v+t v_{0}$. From the definition of $R$ and from (5.8) we deduce that

$$
\begin{aligned}
\eta_{4}(0, v) & =v, \\
\eta_{4}\left(1, K_{3}\right) & =\left\{v_{0}\right\}, \\
\eta_{4}\left([0,1] \times K_{3}\right) & \subset A .
\end{aligned}
$$

The deformations constructed above can be combined and scaled to yield a deformation $\eta \in C\left([0,1] \times \mathcal{S}^{\mathrm{u}}, E\right)$ such that

$$
\begin{aligned}
\eta(0, v) & =v, \\
\eta(1, v) & =v_{0} \in A, \\
\eta\left([0,1] \times \mathcal{S}^{\mathrm{u}}\right) & \subset A .
\end{aligned}
$$


Define the sets

$$
M:=[0,1] \times \mathcal{B}^{\mathrm{u}}, \quad M_{0}:=[0,1] \times \mathcal{S}^{\mathrm{u}} \cup\{0,1\} \times \mathcal{B}^{\mathrm{u}},
$$

the continuous map $\gamma_{0}: M_{0} \rightarrow E$ by

$$
\gamma_{0}(t, v):= \begin{cases}\eta(t, v), & v \in \mathcal{S}^{\mathrm{u}}, \\ v, & t=0, \\ v_{0}, & t=1,\end{cases}
$$

and the class of maps

$$
\Gamma:=\left\{\gamma \in C(M, E):\left.\gamma\right|_{M_{0}}=\gamma_{0}\right\} .
$$

We claim that $M$ and $\mathcal{S}^{\text {s }}$ link, in the following sense:

$$
\forall \gamma \in \Gamma: \gamma(M) \cap \mathcal{S}^{\mathrm{s}} \neq \varnothing .
$$

To see this fix $\gamma \in \Gamma$. Consider the open subset $U:=U_{r} E^{\mathrm{u}}+U_{r} E^{\mathrm{s}}$ of $E$ and define $\kappa_{i}:[0,1] \times \bar{U} \rightarrow E$ for $i=1,2$ by

$$
\begin{aligned}
& \kappa_{1}(t, u):=u^{\mathrm{s}}-u^{\mathrm{u}}+t\left(\xi_{\mathrm{s}}\left(u^{\mathrm{s}}\right)-\xi_{\mathrm{u}}\left(u^{\mathrm{u}}\right)\right), \\
& \kappa_{2}(t, u):=\Xi_{\mathrm{s}}\left(u^{\mathrm{s}}\right)-\gamma\left(t, \Xi_{\mathrm{u}}\left(u^{\mathrm{u}}\right)\right) .
\end{aligned}
$$

Using the fact that $E^{\mathrm{u}}$ is finite dimensional it is easy to see that

$$
\left(I-\kappa_{i}\right)([0,1] \times \bar{U}) \text { is compact for } i=1,2 \text {. }
$$

We will show that the assumption

$$
\gamma(M) \cap \mathcal{S}^{\mathrm{s}}=\varnothing
$$

implies

$$
\kappa_{i}(t, u) \neq 0 \text { for } i=1,2, t \in[0,1] \text { and } u \in \partial U
$$

and

$$
\kappa_{2}(1, u) \neq 0 \text { for } u \in \bar{U} .
$$

Hence, if (5.14) were true, the homotopy invariance of the Leray-Schauder degree, $\kappa_{1}(1, \cdot)=\kappa_{2}(0, \cdot)$, (5.13), (5.15) and (5.16) would imply

$$
\begin{aligned}
0 \neq-1^{\operatorname{dim} E^{\mathrm{u}}} & =\operatorname{deg}\left(P^{\mathrm{s}}-P^{\mathrm{u}}, U, 0\right)=\operatorname{deg}\left(\kappa_{1}(0, \cdot), U, 0\right) \\
& =\operatorname{deg}\left(\kappa_{1}(1, \cdot), U, 0\right)=\operatorname{deg}\left(\kappa_{2}(0, \cdot), U, 0\right)=\operatorname{deg}\left(\kappa_{2}(1, \cdot), U, 0\right)=0,
\end{aligned}
$$

a contradiction.

To prove (5.12) it therefore remains to deduce (5.15) and (5.16) assuming (5.14). By (5.6) and (5.10) $v_{0} \notin \mathcal{B}^{\mathrm{s}}$. This proves (5.16) in view of the definition of $\kappa_{2}, \gamma$ and $\gamma_{0}$. To prove (5.15), always assume that $t \in[0,1]$. If $u \in S_{r} E^{\mathrm{u}}+B_{r} E^{\mathrm{s}}$, then

$$
\left\|P^{\mathrm{u}} \kappa_{1}(t, u)\right\|=\left\|-u^{\mathrm{u}}+t \xi_{\mathrm{s}}\left(u^{\mathrm{s}}\right)\right\| \geq\left\|u^{\mathrm{u}}\right\|-\left\|\xi_{\mathrm{s}}\left(u^{\mathrm{s}}\right)\right\| \geq r-r / 2>0
$$

due to (5.4) and $\xi_{\mathrm{s}}(0)=0$. Similarly it follows that $\kappa_{1}(t, u) \neq 0$ if $u \in B_{r} E^{\mathrm{u}}+S_{r} E^{\mathrm{s}}$. This proves (5.15) for $\kappa_{1}$. If $u \in S_{r} E^{\mathrm{u}}+B_{r} E^{\mathrm{s}}$, then $\gamma\left(t, \Xi_{\mathrm{u}}\left(u^{\mathrm{u}}\right)\right)=\eta\left(t, \Xi_{\mathrm{u}}\left(u^{\mathrm{u}}\right)\right) \in A$. Hence $\kappa_{2}(t, u) \neq 0$ follows from $\Phi\left(\Xi_{\mathrm{s}}\left(u^{\mathrm{s}}\right)\right) \geq 0$ and from the fact that $\Xi_{\mathrm{s}}\left(u^{\mathrm{s}}\right)=0$ if $\Phi\left(\Xi_{\mathrm{s}}\left(u^{\mathrm{s}}\right)\right)=0$. If $u \in B_{r} E^{\mathrm{u}}+S_{r} E^{\mathrm{s}}$, then $\kappa_{2}(t, u) \neq 0$ follows from the assumption (5.14). This proves (5.15) for $\kappa_{2}$ and finishes the proof of (5.12).

As in the proof of [1, Thm. 3.2b)] we now consider a global continuous semiflow $\tilde{\varphi}$, which is just the semiflow $\varphi$ "stopped" at the energy level 0 . This is possible due to Theorem 3.8. Starting with any fixed $\gamma \in \Gamma$ observe that $\tilde{\varphi}(t, \gamma(\cdot)) \in \Gamma$ and 
hence $\tilde{\varphi}(t, \gamma(M)) \cap \mathcal{S}^{\mathrm{s}} \neq \varnothing$ for all $t \geq 0$. Consider a sequence $\left(t_{n}\right)$ with $t_{n} \rightarrow \infty$ as $n \rightarrow \infty$ and $m_{n} \in M$ such that $\tilde{\varphi}\left(t_{n}, \gamma\left(m_{n}\right)\right) \in \mathcal{S}^{\mathrm{s}}$. Since

$$
\Phi\left(\tilde{\varphi}\left(t_{n}, \gamma\left(m_{n}\right)\right)\right) \geq C_{\mathrm{s}}>0
$$

by (5.5) we see that $\tilde{\varphi}\left(t_{n}, \gamma\left(m_{n}\right)\right)=\varphi\left(t_{n}, \gamma\left(m_{n}\right)\right)$. Moreover, it follows that

$$
\Phi\left(\gamma\left(m_{n}\right)\right) \geq \Phi\left(\varphi\left(t_{n}, \gamma\left(m_{n}\right)\right)\right) \geq C_{\mathrm{s}}>0 .
$$

Since $M$ is compact, we may assume that $m_{n} \rightarrow m$ with $\Phi(\gamma(m))>0$. Setting $u:=\gamma(m)$, it is easy to see that this implies $u \in \overline{W^{\mathrm{s}}} \backslash W^{\mathrm{s}}$. The argument used in the proof of [1, Thm 2.4c)] yields an equilibrium in $\overline{W^{\mathrm{s}}} \backslash W^{\mathrm{s}}$ that connects to 0 . Now [1, Thm. 2.5] shows that $\overline{W^{\mathrm{s}}} \backslash\{0\}$ consists entirely of sign changing functions. This finishes the proof.

(b) As in the proof of (a) denote by $E^{\mathrm{s}}$ the generalized eigenspace of $L$ corresponding to the spectral set $\sigma(L) \cap(\lambda, \infty)$ and by $E^{\text {cu }}$ the generalized eigenspace of $L$ corresponding to the spectral set $\sigma(L) \cap\left[\lambda_{1}, \lambda\right]$. Define $W_{\mathrm{loc}}^{\mathrm{s}}, V^{\mathrm{s}}, \xi_{\mathrm{s}} \in C\left(V^{\mathrm{s}}, E^{\mathrm{cu}}\right)$, and $W^{\text {s }}$ similarly as before.

From condition (A7) it follows as in the proof of [30, Thm. 4] that $\Phi \leq 0$ on a neighborhood of 0 in $E^{\mathrm{cu}}$. We may thus choose $r>0$ small enough such that defining $\mathcal{B}^{\mathrm{s}}$ and $\mathcal{S}^{\mathrm{s}}$ as above, and setting

$$
\mathcal{B}^{\mathrm{cu}}:=B_{r} E^{\mathrm{cu}} \quad \text { and } \quad \mathcal{S}^{\mathrm{cu}}:=S_{r} E^{\mathrm{cu}}
$$

it holds that

$$
\Phi(u) \leq 0 \quad \text { for } u \in \mathcal{B}^{\text {cu }} .
$$

Again, put $A:=\Phi^{0} \backslash\{0\}$. Suppose that $\mathcal{K} \cap \Phi^{0}=\varnothing$. Then $\min \left\|\Phi^{\prime}\left(\mathcal{S}^{\mathrm{cu}}\right)\right\|_{H^{-1}}>0$ since $\mathcal{S}^{\mathrm{cu}}$ is compact. The deformation lemma [45, Lemma 2.3] yields

$$
\eta_{0} \in C\left([0,1] \times \mathcal{S}^{\mathrm{cu}}, A\right)
$$

such that setting $K_{0}=\eta_{0}\left(1, \mathcal{S}^{\mathrm{cu}}\right)$ it holds that $\max \Phi\left(K_{0}\right)<0$. Moreover, since by assumption $\varphi$ has no equilibria with negative energy, $\mathcal{O}_{+}(u)$ is unbounded for every $u \in K_{0}$. Replacing $\mathcal{S}^{\mathrm{u}}$ by $K_{0}$ we can proceed exactly as in the proof of (a) to construct a deformation $\eta \in C\left([0,1] \times \mathcal{S}^{\mathrm{cu}}, E\right)$ such that (5.9)-(5.11) are satisfied, and to show the existence of a nodal $u^{*} \in \mathcal{K}$ such that $u^{*}$ connects to 0 .

Remark 5.8. The method used in the preceding proof to construct a homotopical linking at 0 goes back to S. Li. For more information and references see [30].

Proposition 5.9. (a) Let $\bar{u}$ be a strict positive supersolution of $\left(E_{\lambda}\right], \bar{u} \in E$. Then there exists a positive solution $u^{*}$ of $\left(E_{\lambda}\right.$ such that $u^{*} \underset{\mathbf{u}}{\mathrm{u}}$.

(b) Let $\underline{u}$ be a strict negative subsolution of $\left(E_{\lambda}, \underline{u} \in E\right.$. Then there exists a negative solution $u^{*}$ of $\underline{E_{\lambda}}$ such that $u^{*} \geq \underline{u}$.

Proof. We will only prove assertion (a) since the proof of (b) is analogous. Set

$$
D:=\left\{u_{0} \in E: 0<\varphi^{t}\left(u_{0}\right)<\bar{u} \text { for some } t>0\right\} .
$$

Notice that the positive semiorbit $\mathcal{O}_{+}\left(u_{0}\right)$ is global for every $u_{0} \in D$, since the order interval $[0, \bar{u}]$ is positive invariant. In addition, the set $D$ is open. In fact, let $u_{0} \in D, t_{0} \geq 0$ and $0<\varphi^{t_{0}}\left(u_{0}\right)<\bar{u}$. If $t_{1}>t_{0}$, then $\varphi^{t_{1}}\left(u_{0}\right)$ and $\bar{u}-\varphi^{t_{1}}\left(u_{0}\right)$ belong to $\mathcal{P}_{0} X$ due to the maximum principle. Since

$$
\left\|\varphi^{t_{1}}\left(u_{1}\right)-\varphi^{t_{1}}\left(u_{0}\right)\right\|_{X} \leq C\left\|u_{1}-u_{0}\right\|_{E}
$$

for every $u_{1} \in E$ close to $u_{0}$ (where $C=C\left(t_{1}, \max _{0 \leq t \leq t_{1}}\left\|\varphi^{t}\left(u_{0}\right)\right\|_{E}\right)$ ), we see that $u_{1} \in D$ if $\left\|u_{1}-u_{0}\right\|_{E}$ is small enough. 
Fix $\psi \in H_{0}^{1}\left(\Omega^{+}\right), \psi>0$, and set

$$
\alpha^{*}:=\sup A, \quad \text { where } A:=\{\alpha>0: \bar{u}+\alpha \psi \in D\} .
$$

Obviously $\bar{u} \in D$, hence $A$ is a nonempty open set. Since $\Phi$ is bounded below on $[0, \bar{u}]$ and $\Phi(\bar{u}+\alpha \psi) \rightarrow-\infty$ as $\alpha \rightarrow+\infty$ due to Lemma [5.4, we see that $A$ is bounded, $\alpha^{*} \in(0, \infty)$.

The a priori estimates in Section 3 guarantee that the positive semiorbit $\mathcal{O}_{+}\left(\bar{u}+\alpha^{*} \psi\right)$ is global and bounded, hence its $\omega$-limit set is nonempty and consists of equilibria. Choose $u^{*} \in \omega\left(\bar{u}+\alpha^{*} \psi\right)$. Then $u^{*} \geq 0$ due to the maximum principle. Assume that $u^{*} \leq \bar{u}$. Fix $t>0$. Then

$$
u^{*}=\varphi^{t}\left(u^{*}\right) \leq \varphi^{t}(\bar{u}) \ll \bar{u},
$$

which easily implies $\bar{u}+\alpha^{*} \psi \in D$, a contradiction. Consequently, $u^{*} \not \leq \bar{u}$.

Corollary 5.10. We have $\left(-\infty, \lambda^{+}\right) \subset \Lambda^{+}$. In addition, if $\lambda^{+}>\lambda_{1}$ and either meas $\left(\Omega^{0}\right)=0$ or $\lambda^{+} \notin \sigma\left(L_{0}\right)$, then $\lambda^{+} \in \Lambda^{+}$.

Analogous assertions are true for $\lambda^{-}$.

Proof. Let $\lambda<\lambda^{+}$. Then there exists $\bar{\lambda}>\lambda$ such that $\left(E_{\bar{\lambda}}\right)$ possesses a positive solution $\bar{u}$. Since $\bar{u}$ is a strict positive supersolution for $\left(E_{\lambda}\right)$, we have $\lambda \in \Lambda^{+}$by Proposition 5.9 .

Now let $\lambda:=\lambda^{+}>\lambda_{1}$ (notice that (A4) is satisfied with this choice of $\lambda$ ). Consider a sequence $\lambda^{(k)} \nearrow \lambda^{+}$with $\lambda^{(1)}>\lambda_{1}$. Let $e_{1}$ be a positive eigenfunction corresponding to the eigenvalue $\lambda_{1}$ and let $\varepsilon_{0}>0$ be small enough so that $\varepsilon e_{1}$ is a strict subsolution of $\left(E_{\lambda^{(k)}}\right)$ for every $\varepsilon \in\left(0, \varepsilon_{0}\right)$ and every $k$. This guarantees that $\varepsilon_{0} e_{1}<w$ for every positive solution $w$ of $\left(E_{\lambda^{(k)}}\right), k=1,2, \ldots$ The solution of $\left(P_{\lambda^{(k)}}\right)$ with the initial data $u_{0}:=\varepsilon_{0} e_{1}$ converges to the minimal positive solution $w_{k}$ of $\left(E_{\lambda^{(k)}}\right)$, and the energy of $w_{k}$ (corresponding to the problem $\left.\left(P_{\lambda^{(k)}}\right)\right)$ is bounded above by the energy of $u_{0}$. Consequently, the sequence $\left(w_{k}\right)$ is bounded due to Lemma 3.11 (cf. also the proof of Theorem 3.13) (c), hence compact. In addition, $w_{k} \geq \varepsilon_{0} e_{1}$. Thus we may assume that $w_{k} \rightarrow w$, where $w$ is a positive solution of $\left(P_{\lambda}\right)$. This implies $\lambda^{+} \in \Lambda^{+}$.

Corollary 5.11. If $\lambda_{1}<\lambda<\lambda^{+}$, then there exist at least two positive solutions of (E). If $\lambda_{1}<\lambda<\lambda^{-}$, then there exist at least two negative solutions of (E $E_{\lambda}$.

Proof. Let $\lambda \in\left(\lambda_{1}, \lambda^{+}\right)$. Fix $\bar{\lambda} \in\left(\lambda, \lambda^{+}\right)$and let $\bar{u}$ be any positive solution of $\left(E_{\bar{\lambda}}\right)$. Due to Proposition 5.9 we can find a positive solution $u^{*}$ of $\left(E_{\lambda}\right)$ with $u^{*} \not \leq \bar{u}$. On the other hand, choosing $u^{* *} \in \omega(\bar{u})$ we see that $u^{* *}$ is a positive solution of $\left(E_{\lambda}\right)$ (since $0 \notin \omega(\bar{u})$ due to $\lambda>\lambda_{1}$ ) and $u^{* *}<\bar{u}$, hence $u^{* *} \neq u^{*}$.

The proof of the existence of negative equilibria is analogous.

Proposition 5.12. Let $\lambda \leq \lambda^{+}$and let $\bar{u}$ be any positive solution of $\left(E_{\lambda}\right.$ ).

(a) If $\lambda>\lambda_{1}$, then there exists a nodal solution $u^{*}$ of $E_{\lambda}$ such that $u^{*} \not \leq \bar{u}$.

(b) If $\lambda>\lambda_{2}$, then there exists a nodal solution $u^{*}$ of $E_{\lambda}$ such that $u^{*} \leq \bar{u}$ and $0 \succ u^{*}$.

Proof. (a) Define the set $D$ by (5.17). Exactly as in the proof of Proposition 5.9 we can prove that $D$ is open in $E$. Fix $\psi_{1}, \psi_{2} \in H_{0}^{1}\left(\Omega^{+}\right)$linearly independent, $0<\psi_{1}<\bar{u}$, and let $V$ denote the span of $\left\{\psi_{1}, \psi_{2}\right\}$. Similarly as in the proof of Proposition 5.9 we obtain that the set $D \cap V$ is bounded and the positive semiorbit 
$\mathcal{O}_{+}\left(u_{0}\right)$ is global and bounded for every $u_{0} \in M:=\partial(D \cap V)$ (where the boundary is taken in the two-dimensional space $V$ ). Set

$$
\begin{aligned}
& M^{+}:=\left\{u_{0} \in M: u^{*}>0 \text { for every } u^{*} \in \omega\left(u_{0}\right)\right\}, \\
& M^{-}:=\left\{u_{0} \in M: u^{*}<\bar{u} \text { for every } u^{*} \in \omega\left(u_{0}\right)\right\} .
\end{aligned}
$$

Let $u_{0} \in M, u^{*} \in \omega\left(u_{0}\right)$ and $u^{*}<\bar{u}$. Assume that $u^{*}>0$. Then $\bar{u} \gg u^{*} \gg 0$ due to the maximum principle, hence $0<\varphi^{t}\left(u_{0}\right)<\bar{u}$ for some $t>0$, thus $u_{0} \in D$. But $u_{0} \in \partial D$ and $D$ is open, which yields a contradiction. Therefore, $u^{*} \ngtr 0$ and $M^{+} \cap M^{-}=\varnothing$. Next assume that $u^{*}<0$. Then $u^{*} \ll 0$ and one can find $v_{0} \in D$ (close to $u_{0} \in \partial D$ ) such that $\varphi^{t}\left(v_{0}\right)<0$ for some $t>0$ which contradicts the definition of $D$. Hence $u^{*} \nless 0$ and $u^{*}$ has to be either zero or a nodal function. Consequently, $\bar{u} \notin \omega\left(u_{0}\right)$.

Now let $u_{0} \in M, u^{*} \in \omega\left(u_{0}\right)$ and $u^{*}>0$. Then the instability of zero implies $0 \notin \omega\left(u_{0}\right)$. Using these facts it is easy to see that the word "every" in the definitions of $M^{+}, M^{-}$can be replaced by "some". Notice also that the sets $M^{+}, M^{-}$are relatively open in $M$.

Next assume that $M=M^{+} \cup M^{-}$. Fix $\varepsilon \in(0,1)$. Observe that $\varepsilon \psi_{1} \in D \cap V$. We will show that the topological degree $\operatorname{deg}\left(\operatorname{Id}, D \cap V, \varepsilon \psi_{1}\right)$ equals zero, which yields an obvious contradiction. In fact, this assertion can be easily obtained by using the homotopies

$$
\begin{aligned}
& h_{1}(\alpha, u)= \begin{cases}(1-\alpha) u+\alpha \psi_{1}, & u \in M^{+}, \\
(1-\alpha) u-\alpha \psi_{1}, & u \in M^{-},\end{cases} \\
& h_{2}(\alpha, u)= \begin{cases}(1-\alpha) \psi_{1}+\alpha \psi_{2}, & u \in M^{+}, \\
(1-\alpha)\left(-\psi_{1}\right)+\alpha \psi_{2}, & u \in M^{-},\end{cases}
\end{aligned}
$$

where $\alpha \in[0,1]$. Let us check that the homotopy $h_{1}$ is admissible (the admissibility of $h_{2}$ is obvious). Assume on the contrary that $h_{1}(\alpha, u)=\varepsilon \psi_{1}$ for some $u \in M$. Obviously $\alpha \neq 1$. If $u \in M^{+}$, then $u=\psi_{1}(\varepsilon-\alpha) /(1-\alpha)$. Now the definition of $M^{+}$guarantees $\varepsilon>\alpha$ and since $0<(\varepsilon-\alpha) /(1-\alpha)<1$ we see that $u \in D$, a contradiction. If $u \in M^{-}$, then $u=\psi_{1}(\varepsilon+\alpha) /(1-\alpha)>0$ and the instability of zero implies $u^{*}>0$ for every $u^{*} \in \omega(u)$, which contradicts the fact that $M^{+} \cap M^{-}=\varnothing$.

Consequently, $M \neq M^{+} \cup M^{-}$and we can fix $u_{0} \in M \backslash\left(M^{+} \cup M^{-}\right)$. Now every $u^{*} \in \omega\left(u_{0}\right)$ is a nodal solution of $\left(E_{\lambda}\right)$ satisfying $u^{*} \not \leq \bar{u}$.

(b) Let $D$ be defined as above. Let us show that there exists $u_{0} \in \partial D, u_{0} \leq \bar{u}$, lying in the unstable manifold $W_{\text {loc }}^{\mathrm{u}}$ of the zero solution. In fact, $\operatorname{since} \operatorname{dim}\left(W_{\mathrm{loc}}^{\mathrm{u}}\right) \geq$ 2 , there exists a continuous path $\gamma:[-1,1] \rightarrow W_{\text {loc }}^{\mathrm{u}} \backslash\{0\}$ such that $\gamma(-1)<0$, $\gamma(1)>0$ and $\gamma(\alpha)<\bar{u}$ for every $\alpha \in[-1,1]$. Since $\gamma(1) \in D$ and $\gamma(-1) \notin D$, there exists $\alpha_{0} \in[-1,1]$ such that $u_{0}:=\gamma\left(\alpha_{0}\right) \in \partial D$.

Now (similarly as in the proof of (a) we see that the positive semiorbit $\mathcal{O}_{+}\left(u_{0}\right)$ is global and bounded and $\omega\left(u_{0}\right)$ consists of nodal equilibria (due to $u_{0}<\bar{u}$ and $\left.0 \notin \omega\left(u_{0}\right)\right)$. Hence it is sufficient to choose $u^{*} \in \omega\left(u_{0}\right)$.

Proposition 5.13. If $g$ and $h$ are odd in $u$, then there is a sequence $\left(u_{n}\right)$ of nodal equilibria such that $\Phi\left(u_{n}\right) \rightarrow \infty$ as $n \rightarrow \infty$ and such that $u_{n} \succ 0$ for every $n$.

Proof. This can be proved in the same way as [1, Theorem 3.4]. There are two modifications to be made: First, $Y$ should be chosen as a subspace of $H_{0}^{1}\left(\Omega^{+}\right)$now so that Lemma 5.4 can be used. Second, we do not assume (PS) for $\Phi$ in the present 
setting. It needs to be shown: If $A$ is a subset of $\mathcal{K}$ and $\Phi(A)$ is bounded, then $A$ is relatively compact. But here this follows from Theorems 3.2 and 3.7, and from the compactness of the semiflow.

\section{Multiple Equilibria in AN ORDER INTERVAL}

In this section we are concerned with the semiflow $\varphi$ induced on $X$ by the general problem (3.1) in the presence of an attracting order interval. Since we are only interested in the dynamics in the order interval we will not need the a priori estimates and hence no additional growth restrictions or structural conditions on $f$. More precisely, we assume:

$f \in \mathcal{F}_{\mathrm{S}}, f: \Omega \times \mathbb{R} \rightarrow \mathbb{R}$ is differentiable in $u, f_{u}$ is a Carathéodory function, and $f(x, u)-f_{u}(x, 0) u=o(|u|)$ as $|u| \rightarrow 0$, uniformly in $x$.

In particular, 0 is an equilibrium of $\varphi$. We also assume that

there exist a strict subsolution $\underline{u} \ll 0$ of (4.1) and a strict supersolution $\bar{u} \gg 0$ of (4.1) such that $\underline{u}, \bar{u} \in X$,

and we set $C=[\underline{u}, \bar{u}] \subset X$. Then $C$ is closed because $\underline{u}, \bar{u} \in X$, and $C$ has a nonempty interior in $X$.

We define the energy functional $\Phi$ as in Section 3. and we denote the set of nontrivial critical points of $\Phi$ by $\mathcal{K}$. Also denote by $\mu(0)$ the Morse index of 0 with respect to $\Phi$. We are interested in the case when

$$
\mu(0) \geq 2 .
$$

It is easy to see that at a critical point $u$ of $\Phi$ the Morse index of $u$ with respect to the functional $\Phi$ (the dimension of the negative eigenspace of $\Phi^{\prime \prime}(u)$ ) and the dimension of the negative eigenspace of the linearization $-\Delta-f_{u}(x, u(x))$ of (4.1) at $u$ are the same, even though the negative eigenspaces need not coincide. The kernel of $\Phi^{\prime \prime}(u)$ and of $-\Delta-f_{u}(x, u(x))$ always coincide.

Throughout this section we require (6.1), (6.2) and (6.3) to hold. By $e_{1}$ we denote the positive eigenfunction associated with the first eigenvalue of the operator $-\Delta-f_{u}(x, 0)$ in $H_{0}^{1}(\Omega)$. Note that this eigenvalue is negative, hence the statement of Lemma 4.2 is still valid. Therefore in the sequel we fix $\varepsilon_{0}$ such that for all $\varepsilon \in\left(0, \varepsilon_{0}\right]$ the function $\varepsilon e_{1}$ is a strict subsolution and $-\varepsilon e_{1}$ is a strict supersolution of the stationary equation (4.1). This implies, as we have noted in Section 5 , that every positive solution $u$ of (4.1) satisfies $u \gg \varepsilon_{0} e_{1}$, and that every negative solution $u$ of (4.1) satisfies $u \ll-\varepsilon_{0} e_{1}$.

Recall the properties of the semiflow on the order interval $C=[\underline{u}, \bar{u}]$ given by Lemma 4.1. In particular $\Phi$ is bounded below on $C$ and $\varphi^{t}$ restricts to a global semiflow on $C$ (that is, $\varphi^{t}(u)$ is defined for all $t \geq 0$ if $u \in C$ ). Lemma 4.1](b) implies that the following compactness condition holds:

For every sequence $\left(u_{n}\right)$ in $C$ and every sequence $t_{n} \rightarrow \infty$ of real numbers the set $\left\{\varphi^{t_{n}}\left(u_{n}\right): n \in \mathbb{N}\right\} \subset C$ is relatively compact.

Moreover, Lemma 4.1](d) implies that if $u \in C$ is an equilibrium of $\varphi$, then $u \in$ $\operatorname{int}_{X} C$. In particular, $0 \in \operatorname{int}_{X} C$ and hence $W^{\mathrm{u}}(0) \subset C$.

For $\varepsilon \in\left(0, \varepsilon_{0}\right]$ we introduce the order intervals $C_{\varepsilon}^{+}:=\left[\varepsilon e_{1}, \bar{u}\right]$ and $C_{\varepsilon}^{-}:=$ $\left[\underline{u},-\varepsilon e_{1}\right]$, considered as subsets of $X$. By Lemma 4.1 they are strictly positive 
invariant. Let $S^{ \pm}:=\operatorname{inv}\left(C_{\varepsilon}^{ \pm}\right)$be the maximal invariant set. These sets are compact as a consequence of (6.4), and they are not empty because they contain local minimizers $u_{1} \in S^{+}, u_{2} \in S^{-}$. Observe also that there are connecting orbits from 0 to $S^{+}$and $S^{-}$. More precisely $W^{\mathrm{u}}(0) \cap \mathcal{P}_{0} X \neq \varnothing$, and for $u \in W^{\mathrm{u}}(0) \cap \mathcal{P}_{0} X$ we have $\omega(u) \subset S^{+}$. Similarly, $W^{\mathrm{u}}(0) \cap\left(-\mathcal{P}_{0} X\right) \neq \varnothing$ and $\omega(u) \subset S^{-}$for $u \in W^{\mathrm{u}}(0) \cap\left(-\mathcal{P}_{0} X\right)$.

In the sequel we will only consider the restriction of $\varphi$ to $C$. In particular, for a closed subset of $C$ the corresponding set of attraction is taken in $C$. We want to show that there are at least two nodal stationary solutions in $C$, that is, stationary solutions $u_{3}, u_{4} \in C \backslash(\mathcal{P} X \cup(-\mathcal{P} X))$, and we want to find connecting orbits between these solutions and $0, S^{+}, S^{-}$. To this end, define $\mathcal{A}\left(S^{ \pm}\right)$and $\partial \mathcal{A}\left(S^{ \pm}\right)$as in Section 2.1, but here for the restriction of $\varphi$ to $C$. Hence $\partial \mathcal{A}\left(S^{ \pm}\right)$denotes the boundary of $\mathcal{A}\left(S^{ \pm}\right)$relative to $C$. Observe that

$$
( \pm \mathcal{P} X \cap C) \backslash\{0\} \subset \mathcal{A}\left(S^{ \pm}\right) \quad \text { and } \quad 0 \in \partial \mathcal{A}\left(S^{+}\right) \cap \partial \mathcal{A}\left(S^{-}\right) .
$$

Note moreover that

$$
\mathcal{A}\left(S^{ \pm}\right) \text {is open in } C \text { and } \partial \mathcal{A}\left(S^{ \pm}\right) \subseteq \overline{C \backslash \overline{\mathcal{A}\left(S^{ \pm}\right)}} .
$$

We show this for $\mathcal{A}\left(S^{+}\right)$: Since $\mathcal{A}\left(S^{+}\right)$is a neighborhood of $S^{+}$by (6.5) $\mathcal{A}\left(S^{+}\right)$is open in $C$. Now suppose that $u \in \partial \mathcal{A}\left(S^{+}\right)$. Clearly $\mathcal{A}\left(S^{+}\right) \cap(u-\mathcal{P} X)=\varnothing$ since otherwise $\varphi^{t}(u) \in(\mathcal{P} X \cap C) \backslash\{0\} \subseteq \mathcal{A}\left(S^{+}\right)$for large $t$. If $u \in \operatorname{int}_{X} C$ there is

$$
\left(u_{n}\right) \subseteq \operatorname{int}_{X}(u-\mathcal{P} X) \cap C \subseteq C \backslash \overline{\mathcal{A}\left(S^{+}\right)}
$$

such that $u_{n} \rightarrow u$ as $n \rightarrow \infty$. If $u \in \partial_{X} C$, then $\varphi^{t}(u) \in \operatorname{int}_{X} C \cap \partial \mathcal{A}\left(S^{+}\right)$for $t>0$. As above for every $t>0$ there exists a sequence

$$
\left(u_{n}\right) \subseteq \operatorname{int}_{X}\left(\varphi^{t}(u)-\mathcal{P} X\right) \cap C \subseteq C \backslash \overline{\mathcal{A}\left(S^{+}\right)}
$$

such that $u_{n} \rightarrow \varphi^{t}(u)$ as $n \rightarrow \infty$. From this we can build $\left(u_{n}\right) \subseteq C \backslash \overline{\mathcal{A}\left(S^{+}\right)}$such that $u_{n} \rightarrow u$.

Lemma 6.1. $\partial W^{\mathrm{u}}(0) \cap \partial \mathcal{A}\left(S^{ \pm}\right) \neq \varnothing$.

Proof. This can be proved with similar arguments as those used already in the proof of Proposition 5.12. Only note that instead of assuming the a priori estimates from Section 3 here compactness follows since we are working inside the order interval C.

Applying the compactness condition (6.4) once more and using the fact that $\partial W^{\mathrm{u}}(0)$ and $\partial \mathcal{A}\left(S^{ \pm}\right)$are positive invariant we may minimize $\Phi$ on each of the four sets $\partial \mathcal{A}\left(S^{ \pm}\right), \partial \mathcal{A}\left(S^{ \pm}\right) \cap \partial W^{\mathrm{u}}(0)$ and obtain equilibria in $\partial \mathcal{A}\left(S^{ \pm}\right)$and $\partial \mathcal{A}\left(S^{ \pm}\right) \cap$ $\partial W^{\mathrm{u}}(0)$. Since every equilibrium in $\partial W^{\mathrm{u}}(0)$ has negative energy, these minimizers are nontrivial, and by (6.5) they are also nodal. However, they may coincide.

Remark 6.2. Let $u_{3}$ be a (local) minimizer of $\Phi$ on $\partial \mathcal{A}\left(S^{+}\right)$which is also an isolated equilibrium. We shall show that $\lambda_{1}\left(-\Delta-f_{u}\left(x, u_{3}\right)\right) \leq 0 \leq \lambda_{2}\left(-\Delta-f_{u}\left(x, u_{3}\right)\right)$. First note that $u_{3} \in \operatorname{int}_{X} C$. We claim that there exists $\delta>0$ such that $B_{\delta}\left(u_{3}\right) \cap$ $\partial \mathcal{A}\left(S^{+}\right) \subset W^{\mathrm{cs}}\left(u_{3}\right)$. Here $B_{\delta}\left(u_{3}\right):=\left\{u:\left\|u-u_{3}\right\|<\delta\right\}$ and a center-stable manifold $W^{\mathrm{cs}}\left(u_{3}\right)$ is defined as in [12]. (The construction in [12] yields a centerstable manifold in $W^{1, q}$ which we may intersect with $X$ to have a center-stable manifold in $X$.) In order to see this choose $\rho>0$ so that there are no other equilibria in $\overline{B_{\rho}\left(u_{3}\right)}$ except $u_{3}$, and so that $\varphi^{t}(u)$ leaves $\overline{B_{\rho}\left(u_{3}\right)}$ in positive time for 
every $u \in \overline{B_{\rho}\left(u_{3}\right)} \backslash W^{\mathrm{cs}}\left(u_{3}\right)$. This is property (P3) of the center-stable manifold from [12. Assume by contradiction that there exists $v_{n} \in \partial \mathcal{A}\left(S^{+}\right) \backslash W^{\mathrm{cs}}\left(u_{3}\right)$ with $v_{n} \rightarrow u_{3}$ as $n \rightarrow \infty$. Then there exists $t_{n}>0$ with $\left\|\varphi^{t_{n}}\left(v_{n}\right)-u_{3}\right\|=\rho$. Clearly

$$
\lim _{n \rightarrow \infty} \Phi\left(\varphi^{t_{n}}\left(v_{n}\right)\right)=\lim _{n \rightarrow \infty} \Phi\left(v_{n}\right)=\Phi\left(u_{3}\right)
$$

because $u_{3}$ is a minimizer of $\Phi$ on $\partial \mathcal{A}\left(S^{+}\right)$and because $\partial \mathcal{A}\left(S^{+}\right)$is positive invariant. Consequently there exists $s_{n} \in\left[0, t_{n}\right]$ with $\left\|\varphi^{s_{n}}\left(v_{n}\right)-u_{3}\right\| \in[\rho / 2, \rho]$ and $\left.\frac{d}{d t} \Phi\left(\varphi^{t}\left(v_{n}\right)\right)\right|_{t=s_{n}} \rightarrow 0$. This implies $s_{n} \rightarrow \infty$ and $\varphi^{s_{n}}\left(v_{n}\right) \rightarrow v$ along a subsequence by (6.4). It follows that $v$ is an equilibrium and $\left\|v-u_{3}\right\| \in[\rho / 2, \rho]$, contradicting the choice of $\rho$.

Next we claim that the Morse index of $u_{3}$ is at most 1 . In order to see this suppose that $W^{\mathrm{cs}}\left(u_{3}\right)$ has codimension at least 2. Using (6.6) it follows that there exists a path $\gamma:[0,1] \rightarrow B_{\delta}\left(u_{3}\right) \backslash W^{\mathrm{cs}}\left(u_{3}\right)$ with $\gamma(0) \in \mathcal{A}\left(S^{+}\right)$and $\gamma(1) \notin \overline{\mathcal{A}\left(S^{+}\right)}$. Hence there is $s>0$ with $\gamma(s) \in B_{\delta}\left(u_{3}\right) \cap \partial \mathcal{A}\left(S^{+}\right) \subset W^{\mathrm{cs}}\left(u_{3}\right)$, a contradiction.

It is not possible that $u_{3}$ is a strict local minimum for $\Phi$. Assume to the contrary that there exists $\delta>0$ with $\Phi(u)>\Phi\left(u_{3}\right)$ for $u \in B_{\delta}\left(u_{3}\right) \backslash\left\{u_{3}\right\}$. We assume that $B_{\delta}\left(u_{3}\right) \cap S^{+}=\varnothing$ and choose a sequence $v_{n} \in B_{\delta / 2}\left(u_{3}\right) \cap \mathcal{A}\left(S^{+}\right)$converging towards $u_{3}$. From $v_{n} \in \mathcal{A}\left(S^{+}\right)$it follows that there exist $t_{n}>0$ with $\left\|\varphi^{t_{n}}\left(v_{n}\right)-u_{3}\right\|=\delta / 2$. Clearly $t_{n} \rightarrow \infty$ because $v_{n} \rightarrow u_{3}$, and $u_{3}$ is an equilibrium. By (6.4) we may assume that $\varphi^{t_{n}}\left(v_{n}\right) \rightarrow v \in B_{\delta}\left(u_{3}\right) \backslash\left\{u_{3}\right\}$ and $\Phi(v) \leq \liminf _{n \rightarrow \infty} \Phi\left(v_{n}\right)=\Phi\left(u_{3}\right)$, a contradiction.

Thus we have shown that $\lambda_{1}\left(-\Delta-f_{u}\left(x, u_{3}\right)\right) \leq 0 \leq \lambda_{2}\left(-\Delta-f_{u}\left(x, u_{3}\right)\right)$. Generically $u_{3}$ is nondegenerate, hence a critical point of mountain pass type.

From now on we assume that there are only finitely many nodal equilibria in $C$.

Proposition 6.3. If there are only finitely many nodal equilibria in $C$, then there exists a nodal equilibrium $u_{3} \in C$ of mountain pass type and satisfying

$$
\max \left\{\min \Phi\left(S^{+}\right), \min \Phi\left(S^{-}\right)\right\}<\Phi\left(u_{3}\right)<0=\Phi(0) .
$$

Recall that a critical point $u_{0}$ of $\Phi$ is of mountain pass type if for every small open neighborhood $U$ of $u_{0}$ the set $\Phi^{c} \cap U \backslash\left\{u_{0}\right\}, c=\Phi\left(u_{0}\right)$, is not path-connected. This notion has been introduced in [26].

The proof of Proposition 6.3 and the existence of a second nodal equilibrium $u_{4} \in C$ is based on Morse theoretic arguments. This allows for weaker hypotheses and yields more information on the solutions than if working with degree theory; see Remarks 6.5 and 6.6. Let $H_{*}$ denote singular homology theory with coefficients in the field $\mathbb{F}_{2}=\{0,1\}$. In fact, other homology or cohomology theories will work as well. For an isolated critical point $u_{0}$ of $\Phi: E \rightarrow \mathbb{R}$ with $c:=\Phi\left(u_{0}\right)$ the critical groups are defined by

$$
C_{k}\left(\Phi, u_{0}\right):=H_{k}\left(\Phi^{c}, \Phi^{c} \backslash\left\{u_{0}\right\}\right), \quad k \in \mathbb{Z} .
$$

These are in fact $\mathbb{F}_{2}$-vector spaces. Here $\Phi^{c}$ is considered as a subset of $E$. As a consequence of a result of Palais [37, Corollary to Thm. 12] there also holds $C_{k}\left(\Phi, u_{0}\right) \cong H_{k}\left(\Phi^{c} \cap X, \Phi^{c} \cap X \backslash\left\{u_{0}\right\}\right)$. Due to the excision property of homology we have $C_{k}\left(\Phi, u_{0}\right) \cong H_{k}\left(\Phi^{c} \cap U, \Phi^{c} \cap U \backslash\left\{u_{0}\right\}\right)$ for every neighborhood $U$ of $u_{0}$ in $E$ or in $X$; for instance, we may take $U=C$. By [43, Thm. III.4.8] the critical groups are isomorphic to the homology of the Conley index $\mathcal{C}\left(\left\{u_{0}\right\}\right)$ of $\left\{u_{0}\right\}$, considered as an isolated invariant set of the parabolic semiflow:

$$
C_{k}\left(\Phi, u_{0}\right) \cong H_{k}\left(\mathcal{C}\left(\left\{u_{0}\right\}\right)\right) .
$$


Proof of Proposition 6.3. We fix $\varepsilon \in\left(0, \varepsilon_{0}\right]$, so $C_{\varepsilon}^{+}=\left[\varepsilon e_{1}, \bar{u}\right] \subset C$ and $C_{\varepsilon}^{-}=$ $\left[\underline{u},-\varepsilon e_{1}\right] \subset C$ are strictly positive invariant. For $c \in \mathbb{R}$ we set $\Phi_{C}^{c}:=\{u \in C$ : $\Phi(u) \leq c\} \subset C$ and $A^{c}:=\Phi_{C}^{c} \cup C_{\varepsilon}^{+} \cup C_{\varepsilon}^{-}$. For $c<\inf \Phi(C)$ we have $H_{0}\left(A^{c}\right) \cong \mathbb{F}_{2} \oplus \mathbb{F}_{2}$ because $A^{c}=C_{\varepsilon}^{+} \cup C_{\varepsilon}^{-}$is the disjoint union of two convex sets. Since the Morse index of 0 is at least 2 there exists a path $\gamma:[-1,1] \rightarrow W^{\mathrm{u}}(0) \backslash\{0\} \subset C$ with $\gamma( \pm 1) \in C_{\varepsilon}^{ \pm}$. For $c \geq \max _{[-1,1]} \Phi \circ \gamma$ the inclusion

$$
i^{c}: C_{\varepsilon}^{+} \cup C_{\varepsilon}^{-} \hookrightarrow A^{c}
$$

induces a homomorphism

$$
i_{*}^{c}: H_{0}\left(C_{\varepsilon}^{+} \cup C_{\varepsilon}^{-}\right) \rightarrow H_{0}\left(A^{c}\right)
$$

with ker $i_{*}^{c} \neq\{0\}$. Fixing an isomorphism $H_{0}\left(C_{\varepsilon}^{+} \cup C_{\varepsilon}^{-}\right) \cong \mathbb{F}_{2} \oplus \mathbb{F}_{2}$ we may assume that $\operatorname{ker}\left(i_{*}^{c}\right)$ is generated by the element $\alpha \in H_{0}\left(C_{\varepsilon}^{+} \cup C_{\varepsilon}^{-}\right)$corresponding to $(1,-1) \in \mathbb{F}_{2} \oplus \mathbb{F}_{2}$. Now we define

$$
c_{3}:=\inf \left\{c \in \mathbb{R}: i_{*}^{c}(\alpha)=0\right\}=\sup \left\{c \in \mathbb{R}: i_{*}^{c}(\alpha) \neq 0\right\} .
$$

Clearly $c_{3} \leq \max \Phi \circ \gamma<0$. Moreover, $c_{3} \geq \inf \Phi\left(C^{+}\right)$because for $c<\inf \Phi\left(C^{+}\right)$ the set $A^{c}=\Phi_{C}^{c} \cup C_{\varepsilon}^{+} \cup C_{\varepsilon}^{-}$is the disjoint union of the closed subsets $C_{\varepsilon}^{+}$and $\Phi_{C}^{c} \cup C_{\varepsilon}^{-}$. Hence $C_{\varepsilon}^{+}$and $C_{\varepsilon}^{-}$lie in different connected components of $A^{c}$, so $\operatorname{ker}\left(i_{*}^{c}\right)=\{0\}$. Analogously one sees that $c_{3} \geq \inf \Phi\left(C^{-}\right)$.

Next we claim that $H_{1}\left(A^{c_{3}+\delta}, A^{c_{3}-\delta}\right) \neq\{0\}$ for $\delta>0$ small. Consider the commutative diagram:

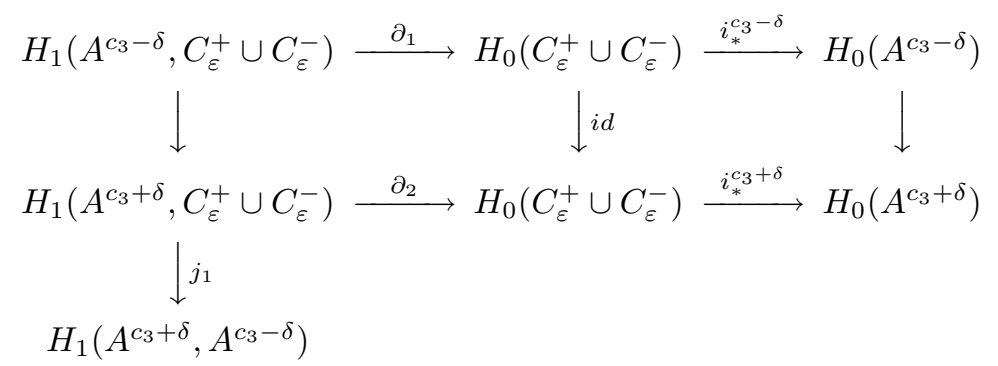

Since $i_{*}^{c_{3}+\delta}(\alpha)=0$ there exists $\beta \in H_{1}\left(A^{c_{3}+\delta}, C_{\varepsilon}^{+} \cup C_{\varepsilon}^{-}\right)$with $\partial_{2}(\beta)=\alpha$ by exactness of the second row. Then $j_{1}(\beta) \neq 0$ because otherwise there exists $\gamma \in H_{1}\left(A^{c_{3}-\delta}, C_{\varepsilon}^{+} \cup C_{\varepsilon}^{-}\right)$with $i_{*}(\gamma)=\beta$; here we used the exactness of the first column. This yields $\partial_{1}(\gamma)=\alpha$ and therefore $i_{*}^{c_{3}-\delta}(\alpha)=i_{*}^{c_{3}-\delta} \circ \partial_{1}(\gamma)=0$, contradicting the definition of $c_{3}$.

It follows that $c_{3}$ is a critical value of $\left.\Phi\right|_{C}$, so there exists an equilibrium in $C$ at the energy level $c_{3}$. In order to see that there exists a nodal equilibrium $u_{3} \in C$ of mountain pass type with $\Phi\left(u_{3}\right)=c_{3}$ we choose $\delta>0$ so that there are no nodal equilibria $u \in C$ with $\Phi(u) \in\left[c_{3}-\delta, c_{3}+\delta\right] \backslash\left\{c_{3}\right\}$. Let $K$ denote the finite set of nodal equilibria $u \in C$ with $\Phi(u)=c_{3}$. Then $K$ equals the set of equilibria in $A^{c_{3}+\delta} \backslash A^{c_{3}-\delta}$ because all signed equilibria from $C$ are contained in $C_{\varepsilon}^{+} \cup C_{\varepsilon}^{-}$. Moreover, the pair $\left(A^{c_{3}+\delta}, A^{c_{3}-\delta}\right)$ is an index pair for $K$ in the sense of [43, and $H_{*}\left(A^{c_{3}+\delta}, A^{c_{3}-\delta}\right)$ is the homological Conley index of $K$. Theorem I.10.4 of [43] yields

$$
\{0\} \neq H_{1}\left(A^{c_{3}+\delta}, A^{c_{3}-\delta}\right) \cong \bigoplus_{u \in K} H_{1}(\mathcal{C}(\{u\}))
$$


Consequently there exists an equilibrium $u_{3} \in K$ with nontrivial critical group $H_{1}\left(\Phi^{c_{3}}, \Phi^{c_{3}} \backslash\left\{u_{3}\right\}\right) \cong H_{1}\left(\Phi_{C}^{c_{3}}, \Phi_{C}^{c_{3}} \backslash\left\{u_{3}\right\}\right) \cong H_{1}\left(\mathcal{C}\left(\left\{u_{3}\right\}\right)\right) \neq\{0\}$. Now [9, Prop. 3.3] states that $u_{3}$ is of mountain pass type.

Proposition 6.4. Suppose that $C_{\mu}(\Phi, 0) \neq 0$ for some $\mu \geq 2$, and that there are only finitely many nodal equilibria in $C$. Then there exists a nodal equilibrium $u_{4} \in C$ with $u_{4} \neq u_{3}$, and one of the following holds:

(i) $\Phi\left(u_{4}\right)<0$ and $C_{\mu-1}\left(\Phi, u_{4}\right) \neq\{0\}$.

(ii) $\Phi\left(u_{4}\right)>0$ and $C_{\mu+1}\left(\Phi, u_{4}\right) \neq\{0\}$.

Remark 6.5. In Proposition 6.4 the origin may be a degenerate equilibrium and may even have trivial local degree. Recall that the local degree $\operatorname{deg}(\nabla \Phi, 0)$ is related to the critical groups via

$$
\operatorname{deg}(\nabla \Phi, 0)=\sum_{k=0}^{\infty}(-1)^{k} \operatorname{dim} C_{k}(\Phi, 0) .
$$

A proof of this can be found in [17, Theorem II.3.2]. The formula holds for $\nabla \Phi: E \rightarrow E$ and the critical groups defined in $E$. (However, since $\nabla \Phi(u)=$ $u-(-\Delta)^{-1}(f(., u))$ we may also work in $X$.) If 0 is a nondegenerate equilibrium with Morse index $\mu$, then $C_{\mu}(\Phi, 0) \cong \mathbb{F}_{2}$.

Remark 6.6. The additional information on the critical groups obtained via our Morse theoretic arguments gives estimates on the index and on the nullity of the solutions. If $\mu\left(u_{4}\right)$ denotes the Morse index of $u_{4}$ and

$$
\nu\left(u_{4}\right)=\operatorname{dim} \operatorname{ker}\left(-\Delta-f_{u}\left(x, u_{4}\right)\right)
$$

is its nullity, then the Shifting Theorem I.5.4 from [17] yields $\mu\left(u_{4}\right) \leq \mu-1 \leq$ $\mu\left(u_{4}\right)+\nu\left(u_{4}\right)$ in case (i) and $\mu\left(u_{4}\right) \leq \mu+1 \leq \mu\left(u_{4}\right)+\nu\left(u_{4}\right)$ in case (ii).

Remark 6.7. Suppose that 0 is a nondegenerate equilibrium with Morse index $\mu$. Then the existence of $u_{4} \neq u_{3}$ with $C_{\mu-1}\left(\Phi, u_{4}\right) \neq\{0\}$ or $C_{\mu+1}\left(\Phi, u_{4}\right) \neq\{0\}$ follows easily from the Morse relations [43, Thm. III.3.5]. In order to see this let $E_{n}$ be the finite set of all nodal equilibria, different from $u_{3}$ (and 0 ). There exists a polynomial $Q(t)$ with nonnegative integer coefficients so that

$$
\begin{gathered}
P\left(t, S^{+}\right)+P\left(t, S^{-}\right)+P\left(t,\left\{u_{3}\right\}\right)+P(t,\{0\})+\sum_{u \in E_{n}} P(t,\{u\}) \\
=P(t, S)+(1+t) Q(t) .
\end{gathered}
$$

Here $P(t, M)$ is the Morse polynomial of the isolated invariant set $M$, and $S:=$ $\operatorname{inv} C$ is the maximal invariant set contained in $C$. An index pair for $S^{+}$is $\left(C_{\varepsilon}^{+}, \varnothing\right)$, so the homology Conley index of $S^{+}$is $H_{k}\left(\mathcal{C}\left(S^{+}\right)\right) \cong \delta_{k 0} \mathbb{F}_{2}$. This yields $P\left(t, S^{+}\right)=$ 1. For the same reasons we have $P\left(t, S^{-}\right)=P(t, S)=1$. Since $u_{3}$ is of mountain pass type [9. Prop. 3.3] implies $H_{k}\left(\mathcal{C}\left(\left\{u_{3}\right\}\right)\right) \cong H_{k}\left(\Phi^{c_{3}}, \Phi^{c_{3}} \backslash\left\{u_{3}\right\}\right) \cong \delta_{k 1} \mathbb{F}_{2}$, where $c_{3}=\Phi\left(u_{3}\right)$. Thus we have $P\left(t,\left\{u_{3}\right\}\right)=t$. Finally, $P(t,\{0\})=t^{\mu}$ by [43, Thm. III.3.5] because 0 is a nondegenerate equilibrium with index $\mu$. Thus we have

$$
1+1+t+t^{\mu}+\sum_{u \in E_{n}} P(t,\{u\})=1+(1+t) Q(t) .
$$

This can only hold if $Q(t)=\sum_{k=0}^{n} a_{k} t^{k}$ satisfies $a_{\mu} \neq 0$ or $a_{\mu-1} \neq 0$. If $a_{\mu} \neq 0$, then there exists $u_{4} \in E_{n}$ so that $P\left(t,\left\{u_{4}\right\}\right)$ has a nontrivial summand $t^{\mu+1}$, hence 
$C_{\mu+1}\left(\Phi, u_{4}\right) \cong H_{\mu+1}\left(\mathcal{C}\left(u_{4}\right)\right) \neq\{0\}$. Similarly, if $a_{\mu-1} \neq 0$ there exists $u_{4} \in E_{n}$ with $C_{\mu-1}\left(\Phi, u_{4}\right) \neq\{0\}$.

Proof of Proposition 6.4. As in the proof of Proposition 6.3 we set $A^{c}=\Phi^{c} \cup C_{\varepsilon}^{+} \cup$ $C_{\varepsilon}^{-}$for $c \in \mathbb{R}$. Let $K_{0}$ denote the finite set of equilibria $u \in C \backslash\left(C_{\varepsilon}^{+} \cup C_{\varepsilon}^{-}\right)$with $\Phi(u)=\Phi(0)=0$. Then we have for $\delta_{1}>0$ small:

$$
H_{\mu}\left(A^{\delta_{1}}, A^{-\delta_{1}}\right) \cong \bigoplus_{u \in K_{0}} H_{\mu}(\mathcal{C}(\{u\})) \supset H_{\mu}(\mathcal{C}(\{0\})) \cong C_{\mu}(\Phi, 0) \neq 0 .
$$

Let $\alpha_{0} \in H_{\mu}\left(A^{\delta_{1}}, A^{-\delta_{1}}\right)$ be a nontrivial element of the summand $H_{\mu}(\mathcal{C}(\{0\}))$. We consider the exact sequence of the pair $\left(A^{\delta_{1}}, A^{-\delta_{1}}\right)$ :

$$
\ldots \rightarrow H_{\mu}\left(A^{\delta_{1}}\right) \stackrel{i_{*}}{\rightarrow} H_{\mu}\left(A^{\delta_{1}}, A^{-\delta_{1}}\right) \stackrel{\partial_{0}}{\rightarrow} H_{\mu-1}\left(A^{-\delta_{1}}\right) \rightarrow \ldots
$$

Case 1. $\partial_{0}\left(\alpha_{0}\right)=0$. Then there exists $\beta_{0} \in H_{\mu}\left(A^{\delta_{1}}\right)$ with $i_{*}\left(\beta_{0}\right)=\alpha_{0}$. For $c \geq \delta_{1}$, let $j^{c}: A^{\delta_{1}} \hookrightarrow A^{c}$ denote the inclusion. For $c$ large enough we have $j_{*}^{c}\left(\beta_{0}\right)=0 \in H_{\mu}\left(A^{c}\right)$. In fact, choose $B \subset A^{\delta_{1}}$ compact so that $\beta_{0}$ lies in the image of $H_{\mu}(B) \rightarrow H_{\mu}\left(A^{\delta_{1}}\right)$. Then $j_{*}^{c}\left(\beta_{0}\right)=0$ for every $c \geq \max \Phi(\operatorname{conv} B)$, conv $B \subset C$ the convex hull.

Now we define

$$
c_{4}:=\sup \left\{c \geq \delta_{1}: j_{*}^{c}\left(\beta_{0}\right) \neq 0\right\}=\inf \left\{c \geq \delta_{1}: j_{*}^{c}\left(\beta_{0}\right)=0\right\} .
$$

It follows easily that for $\delta_{2}>0$ small

$$
H_{\mu+1}\left(A^{c_{4}+\delta_{2}}, A^{c_{4}-\delta_{2}}\right) \neq\{0\}
$$

which yields as in the proof of Proposition 6.3 a nodal equilibrium $u_{4}$ with $\Phi\left(u_{4}\right)=$ $c_{4} \geq \delta_{1}>0$ and $C_{\mu+1}\left(\Phi, u_{4}\right) \neq\{0\}$.

Case 2. $\partial_{0}\left(\alpha_{0}\right) \neq 0$. Let $i_{1}: A^{-\delta_{1}} \hookrightarrow\left(A^{-\delta_{1}}, C_{\varepsilon}^{+} \cup C_{\varepsilon}^{-}\right)$be the inclusion. Since $\mu \geq 2$ and $C_{\varepsilon}^{+} \cup C_{\varepsilon}^{-}$is the disjoint union of two closed convex sets, we have $i_{1 *} \circ \partial_{0}\left(\alpha_{0}\right) \neq 0$ in $H_{\mu-1}\left(A^{-\delta_{1}}, C_{\varepsilon}^{+} \cup C_{\varepsilon}^{-}\right)$. This follows from $H_{k}\left(C_{\varepsilon}^{+} \cup C_{\varepsilon}^{-}\right)=0$ for $k \geq 1$ and the long exact sequence of the pair $\left(A^{-\delta_{1}}, C_{\varepsilon}^{+} \cup C_{\varepsilon}^{-}\right)$.

For $c \leq-\delta_{1}$ let $j^{c}:\left(A^{-\delta_{1}}, C_{\varepsilon}^{+} \cup C_{\varepsilon}^{-}\right) \hookrightarrow\left(A^{-\delta_{1}}, A^{c}\right)$ be the inclusion. Now we define

$$
\begin{aligned}
c_{4} & :=\inf \left\{c \leq-\delta_{1}: j_{*}^{c}\left(i_{1 *} \circ \partial_{0}\left(\alpha_{0}\right)\right)=0\right\} \\
& =\sup \left\{c \leq-\delta_{1}: j_{*}^{c}\left(i_{1 *} \circ \partial_{0}\left(\alpha_{0}\right)\right) \neq 0\right\} .
\end{aligned}
$$

Observe that $j_{*}^{-\delta_{1}}\left(i_{1 *} \circ \partial_{0}\left(\alpha_{0}\right)\right)=0$ and $j_{*}^{c}\left(i_{1 *} \circ \partial_{0}\left(\alpha_{0}\right)\right) \neq 0$ for $c<\inf \Phi(C)$, so $c_{4} \in\left[\inf \Phi(C),-\delta_{1}\right]$. Let

$$
h_{*}^{c}: H_{\mu-1}\left(A^{c}, C_{\varepsilon}^{+} \cup C_{\varepsilon}^{-}\right) \rightarrow H_{\mu-1}\left(A^{-\delta_{1}}, C_{\varepsilon}^{+} \cup C_{\varepsilon}^{-}\right)
$$

be induced by the inclusion. By the definition of $c_{4}$, for $\delta_{2}>0$ small $i_{1 *} \circ \partial_{0}\left(\alpha_{0}\right)=$ $h_{*}^{c_{4}+\delta_{2}}\left(\beta_{0}\right)$ for some $\beta_{0} \in H_{\mu-1}\left(A^{c_{4}+\delta_{2}}, C_{\varepsilon}^{+} \cup C_{\varepsilon}^{-}\right)$but $i_{1 *} \circ \partial_{0}\left(\alpha_{0}\right)$ does not lie in the image of $h_{*}^{c_{4}-\delta_{2}}$. Therefore $\beta_{0}$ does not lie in the image of

$$
H_{\mu-1}\left(A^{c_{4}-\delta_{2}}, C_{\varepsilon}^{+} \cup C_{\varepsilon}^{-}\right) \rightarrow H_{\mu-1}\left(A^{c_{4}+\delta_{2}}, C_{\varepsilon}^{+} \cup C_{\varepsilon}^{-}\right),
$$

hence $0 \neq j_{*}\left(\beta_{0}\right) \in H_{\mu-1}\left(A^{c_{4}+\delta_{2}}, A^{c_{4}-\delta_{2}}\right)$. Here we have denoted the inclusion $j:\left(A^{c_{4}+\delta_{2}}, C_{\varepsilon}^{+} \cup C_{\varepsilon}^{-}\right) \hookrightarrow\left(A^{c_{4}+\delta_{2}}, A^{c_{4}-\delta_{2}}\right)$. As before there exists a nodal equilibrium $u_{4} \in C$ with $\Phi\left(u_{4}\right)=c_{4} \leq-\delta_{1}<0$ and $C_{\mu-1}\left(\Phi, u_{4}\right) \neq\{0\}$.

This proves Proposition 6.4 if $\mu>2$. In the case $\mu=2$ it might be possible that $u_{4}=u_{3}$ and $c_{4}=\Phi\left(u_{4}\right)=\Phi\left(u_{3}\right)=c_{3}$. If that happens to be the case we now show that there exists another nodal equilibrium $u_{4}^{\prime} \in C$ with $\Phi\left(u_{4}^{\prime}\right) \leq c_{4}=c_{3}$. In fact, 
recall from the proof of Proposition 6.3 that there exists $\beta \in H_{1}\left(A^{c_{3}+\delta}, C_{\varepsilon}^{+} \cup C_{\varepsilon}^{-}\right)$ with $\partial_{2}(\beta) \neq 0 \in H_{0}\left(C_{\varepsilon}^{+} \cup C_{\varepsilon}^{-}\right)$and $j_{1}(\beta) \neq 0 \in H_{1}\left(A^{c_{3}+\delta}, A^{c_{3}-\delta}\right), \delta>0$ small. We may assume that $\delta=\delta_{2}$ and $c_{3}+\delta \leq-\delta_{1}$ by choosing $\delta, \delta_{1}$ and $\delta_{2}$ small enough. Let $\partial_{3}: H_{1}\left(A^{-\delta_{1}}, C_{\varepsilon}^{+} \cup C_{\varepsilon}^{-}\right) \rightarrow H_{0}\left(C_{\varepsilon}^{+} \cup C_{\varepsilon}^{-}\right)$be the boundary operator in the exact sequence of the pair $\left(A^{-\delta_{1}}, C_{\varepsilon}^{+} \cup C_{\varepsilon}^{-}\right)$. Then we have $\partial_{2}=\partial_{3} \circ h_{*}^{c_{3}+\delta}$, hence

$$
\partial_{2}\left(\beta_{0}\right)=\partial_{3} \circ h_{*}^{c_{3}+\delta}\left(\beta_{0}\right)=\partial_{3} \circ i_{1 *} \circ \partial_{0}\left(\alpha_{0}\right)=0
$$

because $\partial_{3} \circ i_{1 *}=0$ by exactness. It follows that $\beta_{0} \neq \beta$.

Recall that in an $\mathbb{F}_{2}$-vector space $Y$ two elements $x, y \in Y \backslash\{0\}$ are linearly dependent if and only if $x=y$. If $j_{1}\left(\beta_{0}\right) \neq 0$ and $j_{1}\left(\beta_{0}\right) \neq j_{1}(\beta)$, then $\operatorname{dim} H_{1}\left(A^{c_{3}+\delta}, A^{c_{3}-\delta}\right) \geq 2$. If $u_{3}$ would be the only nodal equilibrium with $\Phi\left(u_{3}\right)=$ $c_{3}$ we would have

$$
H_{1}\left(A^{c_{3}+\delta}, A^{c_{3}-\delta}\right) \cong C_{1}\left(\Phi, u_{3}\right) \cong \mathbb{F}_{2},
$$

a contradiction. It remains to consider the case that $j_{1}\left(\beta_{0}\right)=0$ or $j_{1}\left(\beta-\beta_{0}\right)=0$. Then the exact sequence

$$
H_{1}\left(A^{c_{3}-\delta}, C_{\varepsilon}^{+} \cup C_{\varepsilon}^{-}\right) \rightarrow H_{1}\left(A^{c_{3}+\delta}, C_{\varepsilon}^{+} \cup C_{\varepsilon}^{-}\right) \stackrel{j_{1}}{\longrightarrow} H_{1}\left(A^{c_{3}+\delta}, A^{c_{3}-\delta}\right)
$$

shows that there exists $\gamma_{0} \neq 0 \in H_{1}\left(A^{c_{3}-\delta}, C_{\varepsilon}^{+} \cup C_{\varepsilon}^{-}\right)$. Now we define

$$
c_{4}^{\prime}:=\inf \left\{c \in \mathbb{R}: H_{1}\left(A^{c}, C_{\varepsilon} \cup C_{\varepsilon}^{-}\right) \neq 0\right\}<c_{3}
$$

and conclude as usual that there exists a nodal equilibrium $u_{4}^{\prime} \in C$ with $\Phi\left(u_{4}^{\prime}\right)=c_{4}^{\prime}$ and $C_{1}\left(\Phi, u_{4}^{\prime}\right) \neq 0$.

Remark 6.8. The dynamics of the invariant set $S=\operatorname{inv} C$ can be described in detail if there are no other nodal equilibria in $C$ except $u_{3}, u_{4}$. Suppose that $\Phi\left(u_{4}\right)>0$. First of all, $u_{3}$ is of mountain pass type and lies in $\partial \mathcal{A}\left(S^{+}\right) \cap \partial \mathcal{A}\left(S^{-}\right) \cap \partial W^{\mathrm{u}}(0)$. There exist connecting orbits from 0 to $u_{3}$, from $u_{3}$ to $S^{+}$, and from $u_{3}$ to $S^{-}$. The unstable set $W^{\mathrm{u}}(0)$ contains the relatively open subsets

$$
W_{ \pm}^{\mathrm{u}}(0)=\left\{u \in W^{\mathrm{u}}(0): \varphi^{t}(u) \in C_{\varepsilon}^{ \pm} \text {for } t>0 \text { large }\right\}
$$

which are separated by

$$
W^{\mathrm{u}}(0) \backslash\left(W_{+}^{\mathrm{u}}(0) \cup W_{-}^{\mathrm{u}}(0)\right)=\{0\} \cup\left(W^{\mathrm{u}}(0) \cap W^{\mathrm{s}}\left(u_{3}\right)\right) .
$$

If 0 is nondegenerate with Morse index $\mu \geq 2$, then the set of connecting orbits from 0 to $u_{3}$ has (covering) dimension at least $\mu-1$ because it separates the sets $W^{u}(0) \cap \mathcal{A}\left(S^{+}\right)$and $W^{u}(0) \cap \mathcal{A}\left(S^{-}\right)$which are open subsets of $W^{u}(0)$. There exists a connecting orbit from $u_{4}$ to 0 . If the semiflow is Morse-Smale, then there exists an odd number of connecting orbits. In any case, the complement

$$
W_{c}^{\mathrm{u}}\left(u_{4}\right)=W^{\mathrm{u}}\left(u_{4}\right) \backslash W^{\mathrm{s}}(0)
$$

of the set of connecting orbits from $u_{4}$ to 0 is a relatively open subset of $W^{\mathrm{u}}\left(u_{4}\right)$. It contains the two disjoint sets

$$
W_{ \pm}^{\mathrm{u}}\left(u_{4}\right)=\left\{u \in W^{\mathrm{u}}\left(u_{4}\right): \varphi^{t}(u) \in C_{\varepsilon}^{ \pm} \text {for } t>0 \text { large }\right\}
$$

which are separated by the set $W^{\mathrm{u}}\left(u_{4}\right) \cap W^{\mathrm{s}}\left(u_{3}\right)$ of connecting orbits from $u_{4}$ to $u_{3}$. In particular, this set is nonempty and has $\operatorname{dimension} \operatorname{dim}\left(W^{\mathrm{u}}\left(u_{4}\right) \cap W^{\mathrm{s}}\left(u_{3}\right)\right) \geq \mu$ if $u_{4}$ is nondegenerate. If the flow is Morse-Smale, then this dimension is equal to $\mu$. 
Up to now we proved that at least four nontrivial stationary solutions of (3.1) exist in the order interval $C$. In the following proposition we prove that if $f$ is odd in $u$ there may exist even more nodal equilibria in $C$. In this case we can assume that $\underline{u}=-\bar{u}$, so $C=-C$. We also have

$$
\forall u_{0} \in C, \forall t>0: \varphi^{t}\left(-u_{0}\right)=-\varphi^{t}\left(u_{0}\right), \quad W_{\mathrm{loc}}^{\mathrm{u}}(0)=-W_{\mathrm{loc}}^{\mathrm{u}}(0),
$$

and $P^{\mathrm{u}}: W_{\mathrm{loc}}^{\mathrm{u}}(0) \rightarrow E^{\mathrm{u}}$ is odd. Here $P^{\mathrm{u}}$ is defined as in the proof of Proposition 5.7

Proposition 6.9. Suppose that $f$ is odd in $u$. Then there exist $2(\mu(0)-1)$ nodal equilibria $u_{i}, i= \pm 2, \ldots, \pm \mu(0)$, in $\omega\left(W_{\mathrm{loc}}^{\mathrm{u}}(0)\right)$. In addition, $0 \succ u_{i}, i=$ $\pm 2, \ldots, \pm \mu(0)$.

Proof. First of all we observe that there exists $\rho>0$ such that for $V_{\rho}:=\{u \in E$ : $\left.\left\|P^{\mathrm{u}} u\right\|_{X} \leq \rho\right\}, V_{\rho} \cap W_{\text {loc }}^{\mathrm{u}}(0)$ is $X$-homeomorphic under $P^{\mathrm{u}}$ to $\left\{u \in E^{u}:\|u\| \leq \rho\right\}$. Clearly, all solutions starting from $\partial V_{\rho} \cap W_{\text {loc }}^{\mathrm{u}}(0)$ are global because $\partial V_{\rho} \cap W_{\text {loc }}^{\mathrm{u}}(0) \subset$ $C$.

Due to the compactness of the semiflow generated by (3.1) (see Lemma 4.1) (b) the $\omega$-limit set $\omega(u)$ of $u \in \partial V_{\rho} \cap W_{\text {loc }}^{\mathrm{u}}(0)$ is a nonempty compact and connected set consisting of equilibria. The sets

$$
M_{1}:=\left\{u \in \partial V_{\rho} \cap W_{\text {loc }}^{\mathrm{u}}(0): u^{*}>0 \text { for every } u^{*} \in \omega\left(u_{0}\right)\right\}, \quad M_{-1}:=-M_{1},
$$

are relatively open nonempty subsets of $\partial V_{\rho} \cap W_{\text {loc }}^{\mathrm{u}}(0)$. As already mentioned in the proof of Proposition 5.12 the word "every" may be replaced by the word "some" in the definition of $M_{1}$.

Let us now assume:

there are only finitely many pairs of nodal equilibria $u_{i}$ in $\bigcup\{\omega(u)$ : $\left.u \in V_{\rho} \cap W_{\mathrm{loc}}^{\mathrm{u}}(0)\right\} \subset \partial W^{\mathrm{u}}(0), i= \pm 2, \ldots, \pm m, m \in \mathbb{N}$.

Define

$$
M_{i}:=\left\{u \in \partial V_{\rho} \cap W_{\mathrm{loc}}^{\mathrm{u}}(0): \omega(u)=\left\{u_{i}\right\}\right\}, \quad i= \pm 2, \ldots, \pm m .
$$

These sets satisfy

$$
\bigcup_{i=1}^{m}\left(M_{i} \cup-M_{i}\right)=\partial V_{\rho} \cap W_{\mathrm{loc}}^{\mathrm{u}}(0), \quad M_{-i}=-M_{i}, \quad i=2, \ldots, m .
$$

Since $u_{i}$ is an isolated equilibrium and $\Phi\left(u_{i}\right)=\Phi\left(u_{-i}\right)$ for $i= \pm 2, \ldots, \pm m$, it follows that

$$
M_{i} \cap \bar{M}_{-i}=\varnothing .
$$

A proof can be found in [41, Proof of Theorem 3.1], for instance. Setting

$$
N_{i}:=\partial V_{\rho} \cap W_{\mathrm{loc}}^{\mathrm{u}}(0) \cap \bigcup_{x \in M_{i}}\left\{y:|y-x|<\frac{1}{2} \operatorname{dist}\left(x, \overline{M_{-i}}\right)\right\} \quad i=2, \ldots, m,
$$

we therefore have an open covering

$$
\partial V_{\rho} \cap W_{\mathrm{loc}}^{\mathrm{u}}(0)=M_{1} \cup\left(-M_{1}\right) \cup \bigcup_{i=2}^{m}\left(N_{i} \cup-N_{i}\right)
$$

with $M_{1} \cap-M_{1}=\varnothing, N_{i} \cap-N_{i}=\varnothing$ for $i=2, \ldots, m$. Now we define

$$
O_{i}:= \begin{cases}P^{\mathrm{u}}\left(M_{i}\right), & i=1 \\ P^{\mathrm{u}}\left(N_{i}\right), & i=2, \ldots, m\end{cases}
$$


and $S_{\rho} E^{\mathrm{u}}:=\left\{u \in E^{\mathrm{u}}:\|u\|=\rho\right\}$. The sets $O_{i} \subset S_{\rho} E^{\mathrm{u}}$ are open, $O_{i} \cap-O_{i}=\varnothing$ and $S_{\rho} E^{\mathrm{u}}=\bigcup_{i=1}^{m}\left(O_{i} \cup-O_{i}\right)$ because $P^{\mathrm{u}}: \partial V_{\rho} \cap W_{\text {loc }}^{\mathrm{u}}(0) \rightarrow S_{\rho} E^{\mathrm{u}}$ is a homeomorphism. It is easy to find a closed covering $\bigcup_{i=1}^{m}\left(K_{i} \cup-K_{i}\right)=S_{\rho} E^{\mathrm{u}}, K_{i} \subset O_{i}, i=1, \ldots, m$, hence $m \geq \operatorname{dim}\left(E^{\mathrm{u}}\right) \geq \mu(0)$ (cf. [27, Theorem II.2.7]).

The existence of connecting orbits from 0 to $u_{i}$ follows since $u_{i} \in \omega(u)$ for some $u$ in $V_{\rho} \cap W_{\text {loc }}^{\mathrm{u}}(0)$ by (6.7).

\section{Appendix A. BackWARd uniqueness of the ADJOINT EQUATiOn}

Theorem A.1. Let $\Omega$ be a smoothly bounded domain in $\mathbb{R}^{N}$. Assume that $f: \Omega \times$ $\mathbb{R} \rightarrow \mathbb{R}$ is a Carathéodory function, $f(\cdot, 0) \in L^{\infty}(\Omega)$, the function $f(x, \cdot)$ is continuously differentiable for a.e. $x \in \Omega$ and the derivative satisfies the growth condition

$$
\left|f_{u}(x, u)\right| \leq C\left(1+|u|^{p-1}\right)
$$

for some $p<p_{\mathrm{S}}$. Let $\varphi$ denote the semiflow in $H_{0}^{1}(\Omega)$ generated by the problem

$$
\left\{\begin{aligned}
u_{t}-\Delta u & =f(x, u), & & x \in \Omega, t>0, \\
u & =0, & & x \in \partial \Omega, t>0, \\
u(x, 0) & =u_{0}(x), & & x \in \Omega .
\end{aligned}\right.
$$

Assume that A.1 possesses a (strong) solution on the time interval [0,T]. Then $\varphi^{T}: H_{0}^{1}(\Omega) \rightarrow H_{0}^{1}(\Omega)$ is differentiable at $u_{0}$ and its derivative

$$
D \varphi^{T}\left(u_{0}\right) \in \mathcal{L}\left(H_{0}^{1}(\Omega), H_{0}^{1}(\Omega)\right)
$$

has dense range.

Proof. The differentiability of $\varphi^{T}$ is well known; we will just prove the density assertion.

Step 1. Let $u \in C\left([0, T], H_{0}^{1}(\Omega)\right)$ be the solution of (A.1). It then holds that $u \in C((0, T], C(\bar{\Omega}))$. We will show

$$
|u|^{p-1} \in L^{2}\left((0, T), L^{\rho}(\Omega)\right) \quad \text { for some } \rho>N \text {. }
$$

Due to $u \in L^{\infty}\left((0, T), H_{0}^{1}(\Omega)\right)$ this assertion is obvious for $N \leq 2$ or $p<N /(N-2)$. Hence we may assume $N>2$ and $p \geq N /(N-2)$.

Let $A$ denote the isomorphism $A: H_{0}^{1}(\Omega) \rightarrow H^{-1}(\Omega): u \mapsto-\Delta u$. Observe that the Nemytskii mapping $f^{\#}: H_{0}^{1}(\Omega) \rightarrow H^{-1+\varepsilon}(\Omega): v \mapsto f(\cdot, v)$ is locally Lipschitz continuous for suitable $\varepsilon>0$ small. Consequently, there exists $T_{0}>0$ small such that for all $t_{0} \in\left[0, T_{0}\right]$, the restriction of the solution $u$ to the interval $\left[t_{0}, t_{0}+T_{0}\right]$ can be obtained as a limit of $u_{k} \in C\left(\left[t_{0}, t_{0}+T_{0}\right], H_{0}^{1}(\Omega)\right)$, where

$$
u_{0}(t):=e^{-\left(t-t_{0}\right) A} u\left(t_{0}\right), \quad u_{k+1}(t):=e^{-\left(t-t_{0}\right) A} u\left(t_{0}\right)+\int_{t_{0}}^{t} e^{-(t-\tau) A} f\left(\cdot, u_{k}(\tau)\right) d \tau \text {. }
$$

Set $2^{*}:=2 N /(N-2)$. Since $p<p_{\mathrm{S}}$, there exists $r>N(p-1)$ such that

$$
\alpha:=\frac{N}{2}\left(\frac{1}{2^{*}}-\frac{1}{r}\right)<\frac{1}{2(p-1)} .
$$

Choose $2^{*}=r_{0}<r_{1}<r_{2}<\cdots<r_{k}=r$ such that the numbers

$$
\alpha_{i}:=\frac{N}{2}\left(\frac{1}{r_{i-1}}-\frac{1}{r_{i}}\right), \quad i=1,2, \ldots, k, \quad \beta_{j}:=\frac{N}{2} \cdot \frac{p-1}{r_{j}}, \quad j=0,1,2 \ldots, k,
$$

satisfy

$$
\alpha_{i} p<1, \quad \alpha_{i}(p-1)+\beta_{i}<1, \quad i=1,2, \ldots, k
$$


(notice that such choice is possible due to $\beta_{i} \leq \beta_{0}<1$ ). Fix $t_{*} \in\left(0, T_{0}\right)$ small and set $t_{j}:=j t_{*} / k, j=0,1,2, \ldots, k$. Given

$$
v \in L^{\infty}\left(\left(t_{0}, t_{1}\right), L^{r_{0}}(\Omega)\right) \cap L_{\mathrm{loc}}^{\infty}\left(\left(t_{0}, t_{1}\right), L^{r_{1}}(\Omega)\right),
$$

set

$$
\|v\|_{r_{0}, r_{1}, \alpha_{1}}:=\sup _{t \in\left(t_{0}, t_{1}\right)}\left(\|v(t)\|_{L^{r_{0}}}+\left(t-t_{0}\right)^{\alpha_{1}}\|v(t)\|_{L^{r_{1}}}\right) .
$$

Set $M_{1}:=4\left\|u_{0}\right\|_{L^{2^{*}}}$. We will show that

$$
\left\|u_{k}\right\|_{r_{0}, r_{1}, \alpha_{1}} \leq M_{1}
$$

for all $k$ if $t_{*}=t_{*}\left(M_{1}\right)$ is small enough.

The assertion follows easily from the $L^{p}-L^{q}$ estimates if $k=0$. Next assume that (A.3) is true for some $k \geq 0$. Then

$$
\begin{gathered}
\left(t-t_{0}\right)^{\alpha_{1}}\left\|u_{k+1}(t)\right\|_{L^{r_{1}}} \leq\left\|u\left(t_{0}\right)\right\|_{L^{r_{0}}}+\left(t-t_{0}\right)^{\alpha_{1}} \int_{t_{0}}^{t}(t-\tau)^{-\beta_{1}}\left\|f\left(\cdot, u_{k}(\tau)\right)\right\|_{L^{r_{1} / p}} d \tau \\
\leq M_{1} / 4+C\left(t-t_{0}\right)^{\alpha_{1}} \int_{t_{0}}^{t}(t-\tau)^{-\beta_{1}}\left(1+\left\|u_{k}(\tau)\right\|_{L^{r_{1}}}^{p}\right) d \tau \\
\leq M_{1} / 4+C\left(t-t_{0}\right)^{\alpha_{1}} \int_{t_{0}}^{t}(t-\tau)^{-\beta_{1}}\left(1+\left(\tau-t_{0}\right)^{-\alpha_{1} p} M_{1}^{p}\right) d \tau<M_{1} / 2
\end{gathered}
$$

and

$$
\begin{aligned}
\left\|u_{k+1}(t)\right\|_{L^{r_{0}}} & \leq\left\|u\left(t_{0}\right)\right\|_{L^{r_{0}}}+\int_{t_{0}}^{t}(t-\tau)^{-\beta_{0}}\left\|f\left(\cdot, u_{k}(\tau)\right)\right\|_{L^{r_{0} / p}} d \tau \\
& \leq M_{1} / 4+C \int_{t_{0}}^{t}(t-\tau)^{-\beta_{0}}\left(1+\left\|u_{k}(\tau)\right\|_{L^{r_{0}}}^{p}\right) d \tau \\
& \leq M_{1} / 4+C \int_{t_{0}}^{t}(t-\tau)^{-\beta_{0}}\left(1+M_{1}^{p}\right) d \tau<M_{1} / 2
\end{aligned}
$$

for all $t \in\left(t_{0}, t_{1}\right)$ if $t_{*}$ is small enough, hence (A.3) is true for $u_{k+1}$.

Passing to the limit as $k \rightarrow \infty$ in (A.3) we see that $u$ satisfies the same estimate. Since $u \in C((0, T], C(\bar{\Omega}))$ we have $\left\|u\left(t_{1}\right)\right\|_{L^{r_{1}}} \leq M_{1}\left(t_{1}-t_{0}\right)^{-\alpha_{1}}$. Replacing $t_{0}, t_{1}, r_{0}, r_{1}, \alpha_{1}, \beta_{1}, M_{1}$ with $t_{1}, t_{2}, r_{1}, r_{2}, \alpha_{2}, \beta_{2}$ and $M_{2}:=4 M_{1}\left(t_{1}-t_{0}\right)^{-\alpha_{1}}$ we obtain $\left\|u\left(t_{2}\right)\right\|_{L^{r_{2}}} \leq M_{2}\left(t_{2}-t_{1}\right)^{-\alpha_{2}}$. Repeating this consideration we get $\left\|u\left(t_{*}\right)\right\|_{L^{r}} \leq C t_{*}^{-\alpha}$ for all $t_{*}$ small enough, where $C$ does not depend on $t_{*}$. Consequently, choosing $\rho:=r /(p-1)$ and observing that $2 \alpha(p-1)<1$ we obtain (A.2).

Step 2. Let $f_{1}(t):=f_{u}(\cdot, u(\cdot, t)), v_{0} \in H_{0}^{1}(\Omega)$ and $v \in C\left([0, T], H_{0}^{1}(\Omega)\right)$ be the (strong) solution of the problem

$$
\begin{aligned}
v_{t}+A v & =f_{1}(t) v(t), \quad t \in(0, T], \\
v(0) & =v_{0} .
\end{aligned}
$$

Assume on the contrary that $R:=\left\{v(T): v_{0} \in H_{0}^{1}(\Omega)\right\}$ is not dense in $H_{0}^{1}(\Omega)$. Then there exists $\tilde{z}_{0} \in H_{0}^{1}(\Omega) \backslash\{0\}$ such that $\int_{\Omega} \nabla \tilde{z}_{0} \cdot \nabla w d x=0$ for all $w \in R$, hence

$$
\left(z_{0}, w\right)=0, \quad w \in R
$$

where $z_{0}:=A \tilde{z}_{0} \in H^{-1}(\Omega)$ and by $(\cdot, \cdot)$ we denote both the scalar product in $L^{2}(\Omega)$ and the duality pairing between $H^{-1}(\Omega)$ and $H_{0}^{1}(\Omega)$. 
In Step 3 below we show that there exists a (strong) solution

$$
z \in C\left([0, T], H^{-1}(\Omega)\right) \cap C\left((0, T], H_{0}^{1}(\Omega)\right)
$$

of

$$
\left\{\begin{aligned}
z_{s}+A z & =f_{1}(T-s) z(s), \quad s \in(0, T], \\
z(0) & =z_{0} .
\end{aligned}\right.
$$

Due to the Sobolev maximal regularity, we have

$$
v, z \in W_{\mathrm{loc}}^{1,2}\left((0, T), L^{2}(\Omega)\right) \cap L_{\mathrm{loc}}^{2}\left((0, T), H^{2}(\Omega)\right) .
$$

Since the function $f_{2}:(0, T) \rightarrow L^{2}(\Omega): s \mapsto f_{1}(T-s) z(s)$ is continuous, 44, Theorem II.1.2.2] implies

$$
z \in C\left((0, T), H^{2-\varepsilon}(\Omega)\right) \cap C^{1}\left((0, T), H^{-\varepsilon}(\Omega)\right)
$$

for all $\varepsilon>0$.

Set $y(t):=z(T-t)$. Then $y$ solves

$$
\begin{aligned}
y_{t}-A y & =-f_{1}(t) y(t), \quad t \in[0, T), \\
y(T) & =z_{0},
\end{aligned}
$$

and

$$
\frac{d}{d t}(v(t), y(t))=\left(v_{t}, y\right)+\left(v, y_{t}\right)=\left(-A v+f_{1} v, y\right)+\left(v, A y-f_{1} y\right)=0 \quad \text { a.e. }
$$

Since $t \mapsto(v(t), y(t))$ is absolutely continuous, using (A.4) we obtain

$$
\left(v_{0}, z(T)\right)=(v(0), y(0))=(v(T), y(T))=\left(v(T), z_{0}\right)=0
$$

for all $v_{0} \in H_{0}^{1}(\Omega)$, hence $z(T)=0$. Since $z_{0} \neq 0$, there exists $s_{0}>0$ small such that $z\left(s_{0}\right) \neq 0, z\left(s_{0}\right) \in H_{0}^{1}(\Omega)$. Now we obtain a contradiction from the backward uniqueness for equation (A.5) on $\left[s_{0}, T\right.$ ) using (A.6) and [1, Lemma A.16] with $X_{0}=H^{-\varepsilon}(\Omega), X_{1}=H^{2-\varepsilon}(\Omega) \cap H_{0}^{1}(\Omega), \varepsilon>0$ small. Notice that the assumptions of that lemma are satisfied since A.2 implies $f_{1} \in L^{2}\left((0, T), L^{\rho}(\Omega)\right)$ for some $\rho>N$ and there exists $q<2^{*}$ such that

$$
\begin{aligned}
\left\|f_{1}(T-s) z(s)\right\|_{X_{0}} & \leq C\left\|f_{1}(T-s) z(s)\right\|_{L^{2}} \leq C\left\|f_{1}(T-s)\right\|_{L^{\rho}}\|z(s)\|_{L^{q}} \\
& \leq C\left\|f_{1}(T-s)\right\|_{L^{\rho}}\|z(s)\|_{X_{1 / 2}}
\end{aligned}
$$

if $\varepsilon$ is small enough.

Step 3. It remains to prove the solvability of (A.5). In the proof we will use a (nonlinear) fixed point argument based on similar estimates as in the proof of (A.2) (cf. also 44, 15, 7]). Let us mention that the solvability can also be obtained by "linear methods"; cf. the proof of [6, Lemma 5.1].

First let us prove the existence of a local solution $z \in Z:=C\left(\left[0, s_{0}\right], H^{-1}(\Omega)\right) \cap$ $C\left(\left(0, s_{0}\right], H_{0}^{1}(\Omega)\right)$ satisfying

$$
\|z\|_{Z}:=\sup _{0<s<s_{0}}\left(\|z(s)\|_{H^{-1}}+s^{1 / 2}\|z(s)\|_{L^{2}}+s\|z(s)\|_{H_{0}^{1}}\right)<\infty .
$$

It is known that there exists $C_{A} \geq 1$ such that

$$
\left\{\begin{aligned}
\left\|e^{-s A} w\right\|_{H_{0}^{1}} & \leq C_{A} \min \left\{\|w\|_{H_{0}^{1}}, s^{-1 / 2}\|w\|_{\left.L^{2}, s^{-1}\|w\|_{H^{-1}}\right\}}\right. \\
\left\|e^{-s A} w\right\|_{L^{2}} & \leq C_{A} \min \left\{\|w\|_{L^{2}}, s^{-1 / 2}\|w\|_{H^{-1}}\right\} \\
\left\|e^{-s A} w\right\|_{H^{-1}} & \leq C_{A}\|w\|_{H^{-1}}
\end{aligned}\right.
$$


Let $B_{M}:=\left\{z \in Z:\|z\|_{Z} \leq M\right\}$, where $M=6 C_{A}\left\|z_{0}\right\|_{H^{-1}}$. Given $z \in B_{M}$, define

$$
(G z)(s):=e^{-s A} z_{0}+\int_{0}^{s} e^{-(s-\tau) A} f_{1}(T-\tau) z(\tau) d \tau .
$$

Notice that $\left\|f_{1}(T-\tau)\right\|_{L^{\infty}} \leq C_{f}<\infty$ for $\tau \in\left[0, s_{0}\right]$. Therefore, given $z \in B_{M}$, we have

$$
\begin{aligned}
\left\|e^{-(s-\tau) A} f_{1}(T-\tau) z(\tau)\right\|_{H_{0}^{1}} \leq C_{A}(s-\tau)^{-1 / 2}\left\|f_{1}(T-\tau) z(\tau)\right\|_{L^{2}} \\
\quad \leq C_{A} C_{f}(s-\tau)^{-1 / 2}\|z(\tau)\|_{L^{2}} \leq C_{A} C_{f} M(s-\tau)^{-1 / 2} \tau^{-1 / 2}
\end{aligned}
$$

and

$$
\begin{aligned}
& \left\|e^{-(s-\tau) A} f_{1}(T-\tau) z(\tau)\right\|_{H^{-1}} \leq\left\|e^{-(s-\tau) A} f_{1}(T-\tau) z(\tau)\right\|_{L^{2}} \\
& \leq C_{A}\left\|f_{1}(T-\tau) z(\tau)\right\|_{L^{2}} \leq C_{A} C_{f}\|z(\tau)\|_{L^{2}} \leq C_{A} C_{f} M \tau^{-1 / 2} .
\end{aligned}
$$

Using these estimates and (A.7) it is easy to see that $G: B_{M} \rightarrow B_{M}$ is a contraction (if $s_{0}$ is small enough) and its unique fixed point is the desired solution.

The existence of a prolongation $z \in C\left(\left[s_{0}, T\right], H_{0}^{1}(\Omega)\right)$ follows from the fact that the linear mapping

$$
H_{0}^{1}(\Omega) \rightarrow H^{-1+\varepsilon}(\Omega): z(s) \mapsto f_{1}(T-s) z(s)
$$

is continuous for suitable $\varepsilon>0$, uniformly for $s \in\left[s_{0}, T\right]$. This is due to the fact that $f_{1} \in C\left([0, T], L^{r}(\Omega)\right)$ for some $r>N / 2$.

\section{ACKNOWLEDGEMENT}

Petr Kaplický and Pavol Quittner would like to thank the Institute of Mathematics at the University of Giessen for the hospitality received during their stay in the academic year 2003-2004.

\section{REFERENCES}

1. N. Ackermann and T. Bartsch, Superstable manifolds of semilinear parabolic problems, J. Dynam. Differential Equations 17 (2005), no. 1, 115-173. MR2157843

2. S. Alama and M. Del Pino, Solutions of elliptic equations with indefinite nonlinearities via Morse theory and linking, Ann. Inst. H. Poincaré Anal. Non Linéaire 13 (1996), no. 1, 95-115. MR $96 \mathrm{~m}: 35091$

3. S. Alama and G. Tarantello, On semilinear elliptic equations with indefinite nonlinearities, Calc. Var. Partial Differential Equations 1 (1993), no. 4, 439-475. MR97a:35057

4. H. Amann, Linear and quasilinear parabolic problems, Vol. I abstract linear theory, Monographs in Mathematics, vol. 89, Birkhäuser Boston, Inc., Boston, MA, 1995. MR96g:34088

5. H. Amann and J. López-Gómez, A priori bounds and multiple solutions for superlinear indefinite elliptic problems, J. Differential Equations 146 (1998), no. 2, 336-374. MR99e:35057

6. H. Amann and P. Quittner, Optimal control problems with final observation governed by explosive parabolic equations, SIAM J. Control Optim. 44 (2005), no. 4, 1215-1238 (electronic). MR 2177310

7. J. M. Arrieta, A. N. Carvalho, and A. Rodríguez-Bernal, Parabolic problems with nonlinear boundary conditions and critical nonlinearities, J. Differential Equations 156 (1999), no. 2, 376-406. MR2000f:35075

8. T. Bartsch, Critical point theory on partially ordered Hilbert spaces, J. Funct. Anal. 186 (2001), no. 1, 117-152. MR2002i:58011

9. T. Bartsch, K.-C. Chang, and Z.-Q. Wang, On the Morse indices of sign changing solutions of nonlinear elliptic problems, Math. Z. 233 (2000), no. 4, 655-677. MR2001c:35079

10. T. Bartsch and Z.-Q. Wang, On the existence of sign changing solutions for semilinear Dirichlet problems, Topol. Methods Nonlinear Anal. 7 (1996), no. 1, 115-131. MR97m:35076 
11. T. Bartsch and T. Weth, A note on additional properties of sign changing solutions to superlinear elliptic equations, Topol. Methods Nonlinear Anal. 22 (2003), no. 1, 1-14. MR.2037264

12. P. W. Bates and C. K. R. T. Jones, Invariant manifolds for semilinear partial differential equations, Dynamics reported, Vol. 2, Dynam. Report. Ser. Dynam. Systems Appl., vol. 2, Wiley, Chichester, 1989, pp. 1-38. MR 90g:58017

13. H. Berestycki, I. Capuzzo-Dolcetta, and L. Nirenberg, Superlinear indefinite elliptic problems and nonlinear Liouville theorems, Topol. Methods Nonlinear Anal. 4 (1994), no. 1, 59-78. MR 96d:35041

14. Variational methods for indefinite superlinear homogeneous elliptic problems, NoDEA Nonlinear Differential Equations Appl. 2 (1995), no. 4, 553-572. MR96i:35033

15. H. Brezis and T. Cazenave, A nonlinear heat equation with singular initial data, J. Anal. Math. 68 (1996), 277-304. MR97f:35092

16. T. Cazenave and P.-L. Lions, Solutions globales d'équations de la chaleur semi linéaires, Comm. Partial Differential Equations 9 (1984), no. 10, 955-978. MR87k:35125

17. K.-C. Chang, Infinite-dimensional Morse theory and multiple solution problems, Progress in Nonlinear Differential Equations and their Applications, 6, Birkhäuser Boston, Inc., Boston, MA, 1993. MR94e:58023

18. K.-C. Chang and M.-Y. Jiang, Dirichlet problem with indefinite nonlinearities, Calc. Var. Partial Differential Equations 20 (2004), 257-282. MR2062944 (2005h:35058)

19. W. Chen and C. Li, Indefinite elliptic problems in a domain, Discrete Contin. Dynam. Systems 3 (1997), no. 3, 333-340. MR98a:35035

20. E. N. Dancer and Y. Du, The generalized Conley index and multiple solutions of semilinear elliptic problems, Abstr. Appl. Anal. 1 (1996), no. 1, 103-135. MR97i:35048

21. Yihong Du and Shujie Li, Nonlinear Liouville theorems and a priori estimates for indefinite superlinear elliptic equations, Adv. Differential Equations 10 (2005), no. 8, 841-860. MR 2150868 (2006d:35073)

22. B. Gidas and J. Spruck, A priori bounds for positive solutions of nonlinear elliptic equations, Comm. Partial Differential Equations 6 (1981), no. 8, 883-901. MR82h:35033

23. Y. Giga, A bound for global solutions of semilinear heat equations, Comm. Math. Phys. 103 (1986), no. 3, 415-421. MR87j:35187

24. Y. Giga, S. Matsui, and S. Sasayama, Blow up rate for semilinear heat equation with subcritical nonlinearity, Indiana Univ. Math. J. 53 (2004), no. 2, 483-514. MR2060042 (2005g:35153)

25. M. Grossi, P. Magrone, and M. Matzeu, Linking type solutions for elliptic equations with indefinite nonlinearities up to the critical growth, Discrete Contin. Dynam. Systems 7 (2001), no. 4, 703-718. MR2002h:35086

26. H. Hofer, A geometric description of the neighbourhood of a critical point given by the mountain-pass theorem, J. London Math. Soc. (2) 31 (1985), no. 3, 566-570. MR87e:58041

27. M. A. Krasnosel'skii, Topological methods in the theory of nonlinear integral equations, Translated by A. H. Armstrong; translation edited by J. Burlak. A Pergamon Press Book, The Macmillan Co., New York, 1964. MR28:2414

28. H. A. Levine, Some nonexistence and instability theorems for solutions of formally parabolic equations of the form $P u_{t}=-A u+\mathcal{F}(u)$, Arch. Rational Mech. Anal. 51 (1973), 371-386. MR 50:714

29. S. Li and Z.-Q. Wang, Mountain pass theorem in order intervals and multiple solutions for semilinear elliptic Dirichlet problems, J. Anal. Math. 81 (2000), 373-396. MR2001h:35063

30. S. Li and M. Willem, Applications of local linking to critical point theory, J. Math. Anal. Appl. 189 (1995), no. 1, 6-32. MR 96a:58045

31. J. López-Gómez and P. Quittner, Complete and energy blow-up in indefinite superlinear parabolic problems, Discrete Contin. Dyn. Syst. 14 (2006), no. 1, 169-186. MR2170308

32. P. Magrone, On a class of semilinear elliptic equations with potential changing sign, Dynam. Systems Appl. 9 (2000), no. 4, 459-467. MR2002d:35070

33. M. Marcus and L. Véron, Uniqueness and asymptotic behavior of solutions with boundary blow-up for a class of nonlinear elliptic equations, Ann. Inst. H. Poincaré Anal. Non Linéaire 14 (1997), no. 2, 237-274. MR.97m:35068

34. H. Matano, Existence of nontrivial unstable sets for equilibriums of strongly order-preserving systems, J. Fac. Sci. Univ. Tokyo Sect. IA Math. 30 (1984), no. 3, 645-673. MR.85d:35014

35. J. Mawhin and M. Willem, Critical point theory and Hamiltonian systems, Applied Mathematical Sciences, vol. 74, Springer-Verlag, New York, 1989. MR.90e:58016 
36. W.-M. Ni, P. E. Sacks, and J. Tavantzis, On the asymptotic behavior of solutions of certain quasilinear parabolic equations, J. Differential Equations 54 (1984), no. 1, 97-120. MR $87 \mathrm{j}: 35057$

37. R. S. Palais, Homotopy theory of infinite dimensional manifolds, Topology 5 (1966), 1-16. MR.32:6455

38. P. Quittner, Global existence of solutions of parabolic problems with nonlinear boundary conditions, Singularities and differential equations (Warsaw, 1993), Banach Center Publ., vol. 33, Polish Acad. Sci., Warsaw, 1996, pp. 309-314. MR98h:35127

39. - A priori bounds for global solutions of a semilinear parabolic problem, Acta Math. Univ. Comenian. (N.S.) 68 (1999), no. 2, 195-203. MR.2001d:35026

40. Continuity of the blow-up time and a priori bounds for solutions in superlinear parabolic problems, Houston J. Math. 29 (2003), no. 3, 757-799 (electronic). MR.1998164

41. _ Multiple equilibria, periodic solutions and a priori bounds for solutions in superlinear parabolic problems, NoDEA Nonlinear Differential Equations Appl. 11 (2004), 237-258. MR.2210288 (2006k:35140)

42. M. Ramos, S. Terracini, and C. Troestler, Superlinear indefinite elliptic problems and Pohožaev type identities, J. Funct. Anal. 159 (1998), no. 2, 596-628. MR2000h:35053

43. K. P. Rybakowski, The homotopy index and partial differential equations, Universitext, Springer-Verlag, Berlin, 1987. MR89d:58025

44. F. B. Weissler, Semilinear evolution equations in Banach spaces, J. Funct. Anal. 32 (1979), no. 3, 277-296. MR80i:47091

45. M. Willem, Minimax theorems, Progress in Nonlinear Differential Equations and their Applications, 24, Birkhäuser Boston, Inc., Boston, MA, 1996. MR.97h:58037

Instituto de Matemáticas, Universidad Nacional Autónoma de México, México, D.F. C.P. 04510, MÉXICO

E-mail address: nils@ackermath.info

Mathematisches Institut, University of Giessen, Arndtstr. 2, 35392 Giessen, GerMANY

E-mail address: thomas.bartsch@math.uni-giessen.de

Faculty of Mathematics and Physics, Charles University Prague, Sokolovská 83, 186 75 Praha 8, Czech Republic

E-mail address: kaplicky@karlin.mff.cuni.cz

Department of Applied Mathematics and Statistics, Comenius University, Mlynská DOLINA, 84248 BRATiSlaVA, SLOVAKIA

E-mail address: quittner@pc2.iam.fmph.uniba.sk 